\title{
A CONSTANT CONDUCTION ANGLE BIASED RF POWER AMPLIFIER FOR IMPROVED LINEARIZATION IN CLASS C OPERATION
}

\author{
A Thesis presented to \\ the Faculty of California Polytechnic State University, \\ San Luis Obispo
}

\author{
In Partial Fulfillment \\ of the Requirements for the Degree \\ Master of Science in Electrical Engineering \\ by \\ Greg LaCaille \\ June 2010
}


(C) 2010

Greg LaCaille

ALL RIGHTS RESERVED 


\section{COMMITTEE MEMBERSHIP}

TITLE:

AUTHOR:

DATE SUBMITTED:

COMMITTEE CHAIR:

COMMITTEE MEMBER:

COMMITTEE MEMBER:
A Constant Conduction Angle Biased RF Power Amplifier for Improved Linearization in Class C Operation

Greg LaCaille

June 2010

Vladimir Prodanov, Professor of Electrical Engineering, Cal Poly

Cheng Sun, Professor of Electrical Engineering, Cal Poly

Tina Smilkstein, Professor of Electrical Engineering, Cal Poly 


\begin{abstract}
A Constant Conduction Angle Biased RF Power Amplifier for Improved

Linearization in Class C Operation

Greg LaCaille
\end{abstract}

Class $C$ power amplifiers offer higher efficiency than class $B$ power amplifiers, but suffer from poor linearity. A feedback based biasing system to improve the linearity of a class $C$ power amplifier is designed. A class $B$ amplifier with a gain of $20 \mathrm{~dB}$ and $20 \mathrm{MHz}$ bandwidth at $900 \mathrm{MHz}$ acts as the launching point for the design. The biasing and output network of the class $\mathrm{B}$ power amplifier is modified to produce a class $\mathrm{C}$ amplifier at conduction angles of $180^{\circ}, 162^{\circ}, 126^{\circ}, 90^{\circ}$, and $54^{\circ}$. A feedback based biasing system, which uses two matched and scaled down transistors, compares the DC current of a class B and a class $C$ biased transistor. This comparison is used to control the biasing voltage of the amplifier. The performance for each class $C$ amplifier is simulated with the proposed constant conduction angle biasing (CCAB) system. The conduction angle, transducer gain, operational gain, VSWR, and drain efficiency are measured from simulation for each of the 5 normally biased and 5 CCAB amplifiers. Dynamic ranges of over $8 \mathrm{~dB}$ are demonstrated for the CCAB amplifiers. The effects of loop gain, temperature, and operating frequency for the $126^{\circ}$ amplifier are simulated. The $3^{\text {rd }}$ order intermodulation products of a $10 \mathrm{MHz}$ AM modulated $900 \mathrm{MHz}$ signal are compared for the $126^{\circ}$ normally biased and CCAB biased amplifier as well as the class $B$ amplifier. The difference between the fundamental and the $3^{\text {rd }}$ order intermodulation products is shown to improve from $9.9 \mathrm{~dB}$ for the normal class $\mathrm{C}$ to $28.7 \mathrm{~dB}$ for the CCAB amplifier. 


\section{Table of Contents}

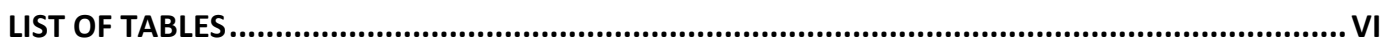

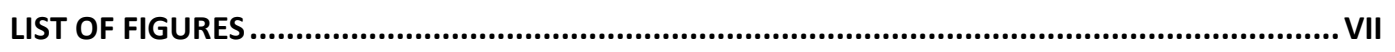

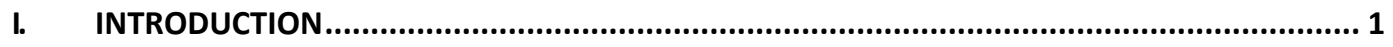

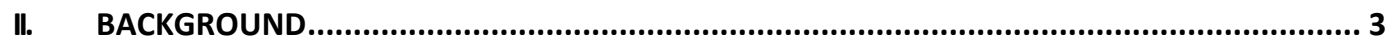

III. EFFECT OF CONDUCTION ANGLE FOR PIECEWISE TRANSFER CHARACTERISTIC ................. 5

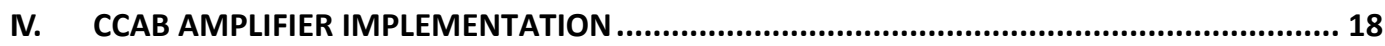

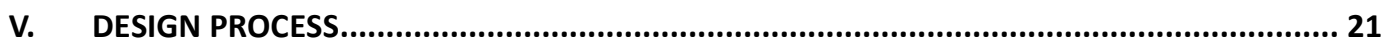

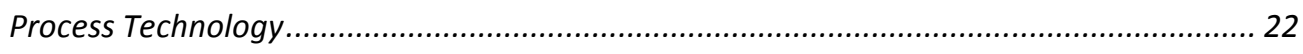

Transistor Characterization ........................................................................................ 23

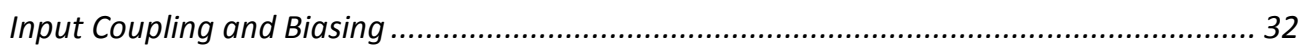

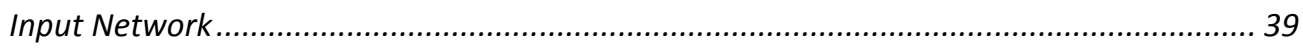

Output Matching Network ........................................................................................ 40

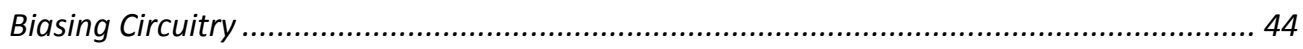

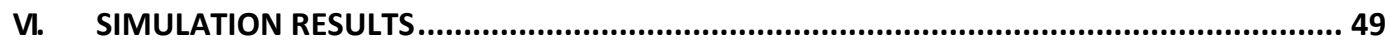

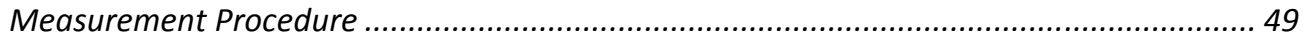

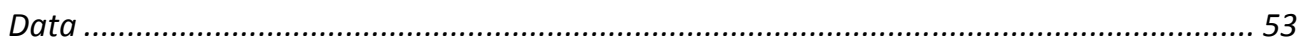

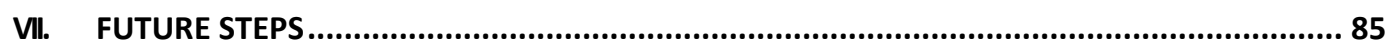

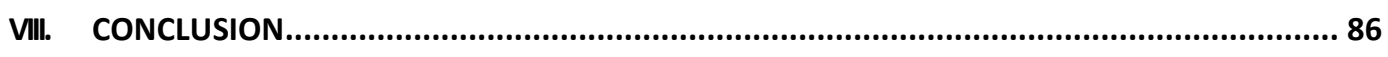

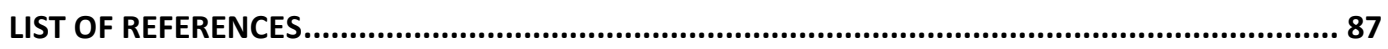




\section{List of Tables}

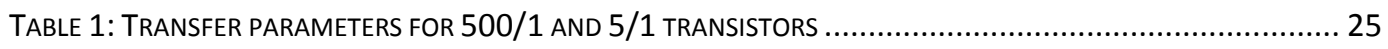

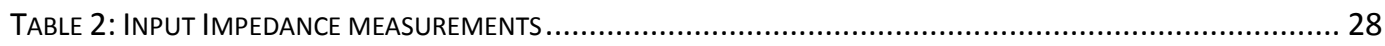

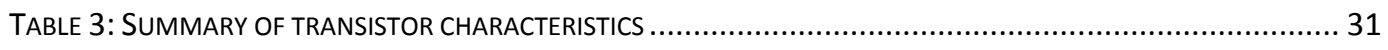

TABLE 4: DESIRED OUTPUT RESISTANCE FOR MULTIPLE CONDUCTION ANGLES .............................................. 41

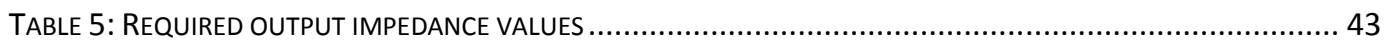

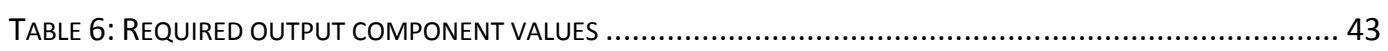

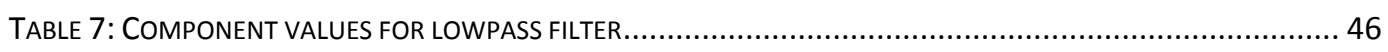

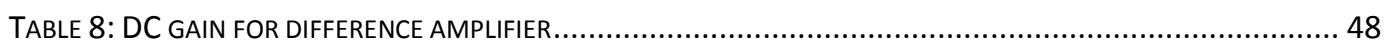




\section{List of Figures}

FIGURE 1: VOLTAGE AND CURRENT WAVEFORMS FOR PIECEWISE APPROXIMATION ..........................................

FIGURE 2: GAIN AS A FUNCTION OF CONDUCTION ANGLE, $R_{\text {OUT }}$ SET FOR MAX SWING ....................................

FIGURE 3: GAIN RELATIVE TO CLASS B OPERATION AS A FUNCTION OF CONDUCTION ANGLE, $R_{\text {OUT }}$ SET FOR

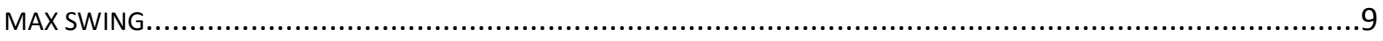

FigURE 4: GAIN RELATIVE TO CLASS B OPERATION, IN DB, AS A FUNCTION OF CONDUCTION ANGLE, $R_{\text {oUt }}$

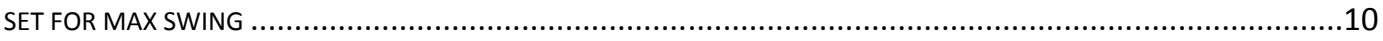

FIGURE 5: EFFICIENCY AS A FUNCTION OF CONDUCTION ANGLE, R RUT SET FOR MAX SWING ................................11

Figure 6: EfFICIENCY RELATIVE to CLASS B OPERATION AS A FUNCTION OF CONDUCTION ANGLE, RoUT SET

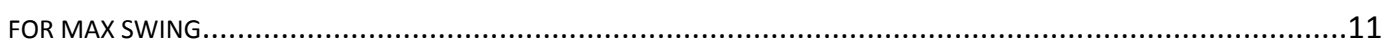

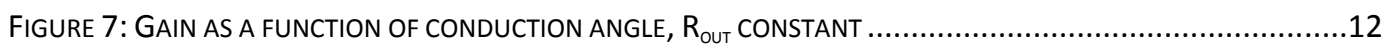

FIGURE 8: GAIN RELATIVE TO CLASS B OPERATION AS A FUNCTION OF CONDUCTION ANGLE, $R_{\text {OUt }}$ CONSTANT ...........13

Figure 9: GAIN RELATIVE TO CLASS B OPERATION, IN DB, AS A FUNCTION OF CONDUCTION ANGLE, $R_{\text {OUt }}$

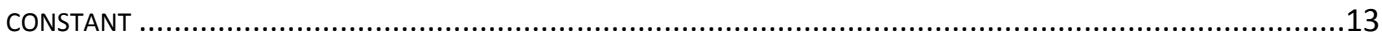

FIGURE 10: EFFICIENCY AS A FUNCTION OF CONDUCTION ANGLE, $R_{\text {OUT }}$ CONSTANT ...........................................14

FIGURE 11: EFFICIENCY RELATIVE TO CLASS B OPERATION AS A FUNCTION OF CONDUCTION ANGLE, $R_{\text {OUT }}$

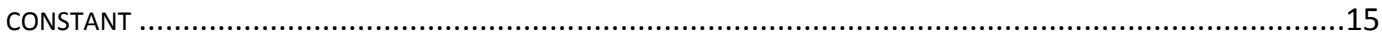

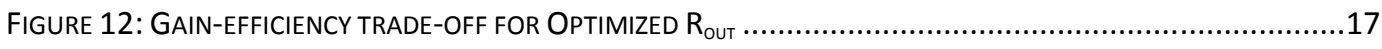

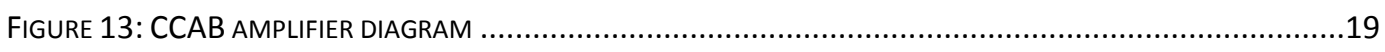

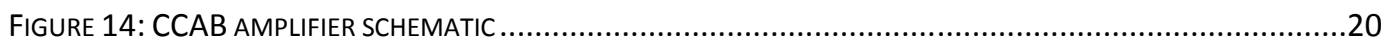

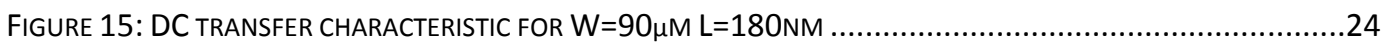

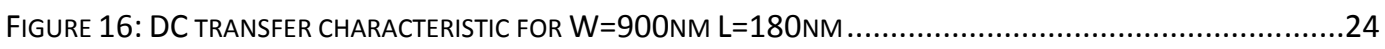

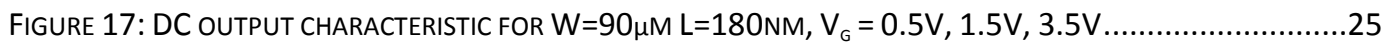

FIGURE 18: DC OUTPUT CHARACTERISTIC FOR W=900NM L=180NM, $V_{G}=0.5 \mathrm{~V}, 1.5 \mathrm{~V}, 3.5 \mathrm{~V} \ldots \ldots \ldots \ldots \ldots \ldots \ldots . . .26$

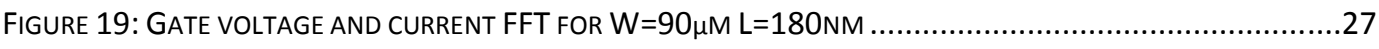

FIGURE 20: GATE VOLTAGE AND CURRENT FFT FOR W=900NM L=180NM ...........................................27 
FIGURE 21: SMALL SIGNAL INPUT REACTANCE AS A FUNCTION OF GATE BIAS FOR W=90 $\mu \mathrm{M}$ L=180NM.

FIGURE 22: SMALL SIGNAL INPUT REACTANCE AS A FUNCTION OF GATE BIAS FOR W=900NM L=180NM ............29

FIGURE 23: SMALL SIGNAL OUTPUT IMPEDANCE AS A FUNCTION OF GATE BIAS FOR W=90 $\mu \mathrm{M}$ L=180NM ............30

FIGURE 24: SMALL SIGNAL OUTPUT IMPEDANCE AS A FUNCTION OF GATE BIAS FOR W=900NM L=180NM ...........30

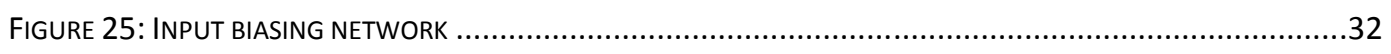

FIGURE 26: VolTAGE DIVIDER RATIO AS A FUNCTION OF POLE TO BANDWIDTH RATIO ......................................3

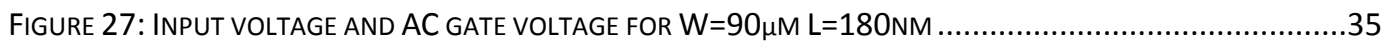

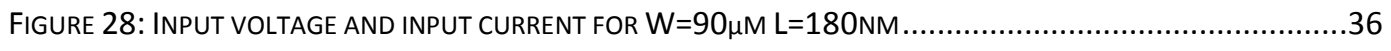

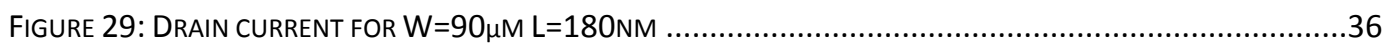

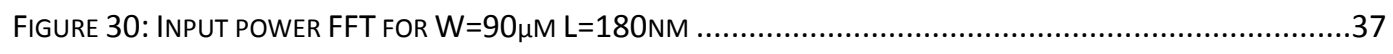

FIGURE 31: INPUT VOLTAGE AND AC GATE VOLTAGE FOR W=900NM L=180NM ......................................38

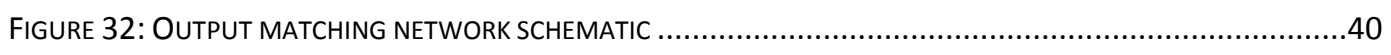

FIGURE 33: OPERATIONAL GAIN OF CLASS B AMPLIFIER FOR DIFFERENT BIAS VOLTAGES ..................................44

FIGURE 34: CONDUCTION ANGLE OF CLASS B AMPLIFIER FOR DIFFERENT BIAS VOLTAGES .................................45

FIGURE 35: CLASS C BIAS VOLTAGE FOR DIFFERENT ANGLE SETTINGS ...................................................4

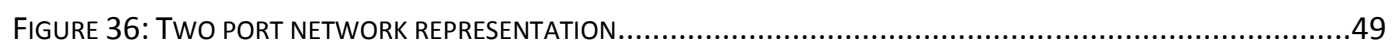

FIGURE 37: DEFINITION FOR T

FIGURE 38: CONDUCTION ANGLE FOR NB AMPLIFIERS OVER 40 DB RANGE ........................................53

FIGURE 39: CONDUCTION ANGLE FOR NB AMPLIFIERS OVER 20 DB RANGE ..........................................5

FIGURE 40: CONDUCTION ANGLE FOR CCAB AMPLIFIERS OVER 40 DB RANGE.........................................55

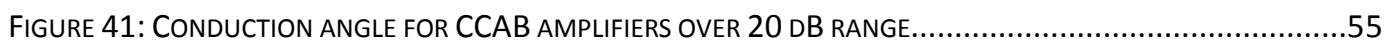

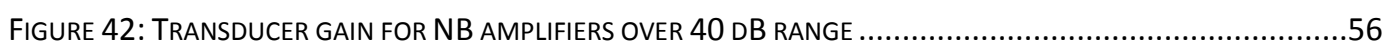

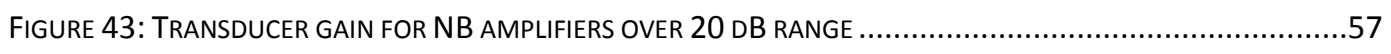

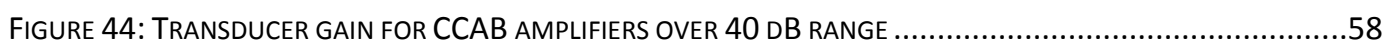

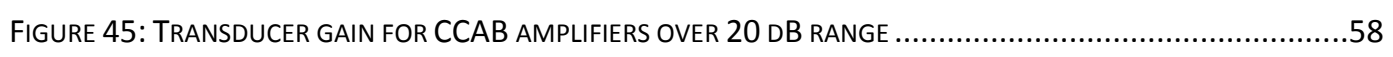

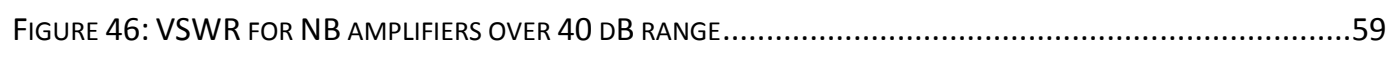

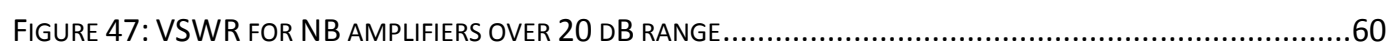


FIGURE 48: VSWR FOR CCAB AMPLIFIERS OVER 40 DB RANGE

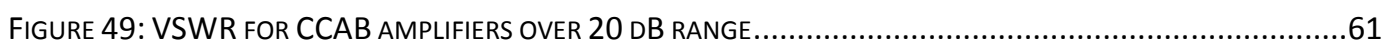

FIGURE 50: OPERATIONAL GAIN FOR NB AMPLIFIERS OVER 40 DB RANGE ..............................................63

FIGURE 51: OPERATIONAL GAIN FOR NB AMPLIFIERS OVER 20 DB RANGE ...............................................63

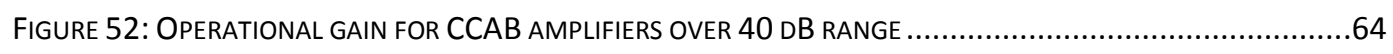

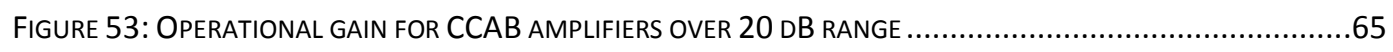

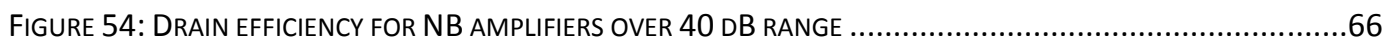

FIGURE 55: DRAIN EFFICIENCY FOR NB AMPLIFIERS OVER 20 DB RANGE ..........................................66

FIGURE 56: DRAIN EFFICIENCY FOR CCAB AMPLIFIERS OVER 40 DB RANGE ............................................67

FIGURE 57: DRAIN EFFICIENCY FOR CCAB AMPLIFIERS OVER 20 DB RANGE ..........................................68

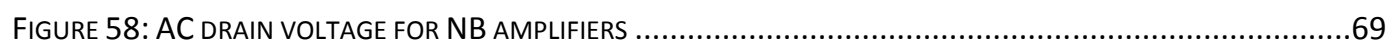

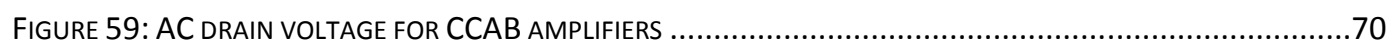

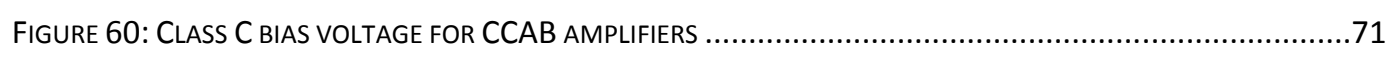

FIGURE 61: CLASS C BIAS VOLTAGE AT $126^{\circ}$ SETTING FOR MULTIPLE VALUES OF K .......................................72

FIGURE 62: CONDUCTION ANGLE OF $126^{\circ}$ CCAB AMPLIFIER FOR MULTIPLE VALUES OF K ..............................73

FIGURE 63: TRANSDUCER GAIN OF $126^{\circ}$ CCAB AMPLIFIER FOR MULTIPLE VALUES OF K ...............................74

FIGURE 64: OPERATIONAL GAIN OF $126^{\circ}$ CCAB AMPLIFIER FOR MULTIPLE VALUES OF K .................................75

FIGURE 65: CONDUCTION ANGLE OF $126^{\circ}$ CCAB AMPLIFIER FOR MULTIPLE TEMPERATURES...........................76

FIGURE 66: TRANSDUCER GAIN OF $126^{\circ}$ CCAB AMPLIFIER FOR MULTIPLE TEMPERATURES .............................77

FIGURE 67: OPERATIONAL GAIN OF $126^{\circ}$ CCAB AMPLIFIER FOR MULTIPLE TEMPERATURES .............................78

FIGURE 68: CONDUCTION ANGLE OF $126^{\circ}$ CCAB AMPLIFIER FOR MULTIPLE VALUES OF P $\mathrm{P}_{\text {INC }}$ OVER $2.3 \mathrm{GHZ}$

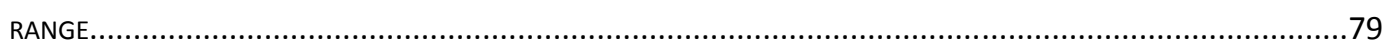

FIGURE 69: CONDUCTION ANGLE OF $126^{\circ}$ CCAB AMPLIFIER FOR MULTIPLE VALUES OF $P_{\text {INC }}$ OVER 200

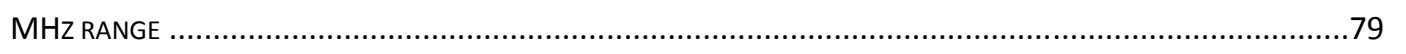

FIGURE 70: TRANSDUCER GAIN OF $126^{\circ}$ CCAB AMPLIFIER FOR MULTIPLE VALUES OF $\mathrm{P}_{\text {INC }}$ OVER $2.3 \mathrm{GHZ}$

RANGE... 
FiguRE 71: TRANSDUCER GAIN OF $126^{\circ}$ CCAB AMPLIFIER FOR MULTIPLE VALUES OF $P_{\text {INC }}$ OVER $200 \mathrm{MHz}$

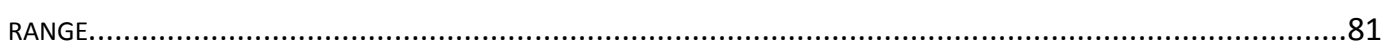

FIGURE 72: VSWR OF $126^{\circ}$ CCAB AMPLIFIER FOR MULTIPLE VALUES OF $P_{\text {INC }}$ OVER 200 MHZ RANGE ................82

FIGURE 73: OUTPUT POWER OF FUNDAMENTAL AND $3^{\text {RD }}$ ORDER INTERMODULATION PRODUCTS FOR $0.7 \mathrm{~V}$

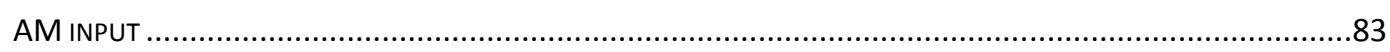

FIGURE 74: DIFFERENCE IN OUTPUT POWER OF FUNDAMENTAL AND $3^{\text {RD }}$ ORDER INTERMODULATION

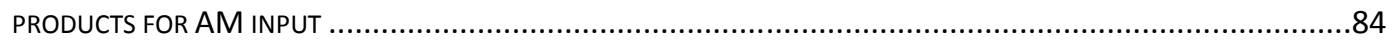




\section{Introduction}

When designing a RF communication system the choice of modulation technique will have a significant impact on many areas of performance for the system. For long range wireless communications, large amounts of power may be required to overcome loss in the channel. Typically efficiency will be a high priority in the design for these systems, since a small decrease in efficiency will still translate to a large amount of power wasted. This often means that a phase or frequency based modulation scheme will be used, since a constant magnitude allows for the use of very efficient switching power amplifiers. ${ }^{1}$ One example of this is the GSM cellular standard which uses Gaussian minimum shift keying. ${ }^{2}$ On the other hand, using amplitude modulation in conjunction with frequency or phase modulation can achieve higher data rates by more effectively utilizing the bandwidth. The LTE wireless standard uses QAM64 modulation. ${ }^{3}$ With the need for amplitude modulated wireless communications, power amplifiers capable of dealing with the high peak to average power ratio (PAPR) that run efficiently are required. Many techniques have been researched to allow use of efficient switching power amplifiers in these applications. However, little research has been done on techniques to improve the effectiveness of the linear amplifier classes. This paper investigates the effectiveness of using feedback to dynamically adjust the bias of a class $C$ amplifier. This proposed constant conduction 
angle bias (CCAB) system should offer improved linearity, but still realize the efficiency typical of class $\mathrm{C}$ amplifiers. 


\section{Background}

Class $\mathrm{A}, \mathrm{B}$, and $\mathrm{C}$ amplifiers maintain only the fundamental harmonic in the voltage at the drain of the transistor. Class A amplifiers also contain only the fundamental harmonic in the current as well. Class B amplifiers only conduct for $50 \%$ of the cycle. This introduces even harmonics into the drain current. However the matching network only maintains only the fundamental in the voltage waveform so no power is transmitted in the upper harmonics. Class $C$ amplifiers conduct less than $50 \%$ of the cycle. A truly ideal class C amplifier would have an impulse of current at the peak of conduction. This periodic impulse of current contains both odd and even harmonics. The period that the transistor conducts is typically referred to as the conduction angle. ${ }^{4}$

Class D, E, and F amplifiers operate as switching amplifiers. Because these amplifiers use the transistor as a switch, which only transmits power through an on/off manner, amplitude modulation cannot be applied to switching PAs. However, because the zero crossing of these amplifiers is unchanged frequency or phase modulation remains intact. ${ }^{5}$

Since switching PAs are not suitable for amplitude modulated signals many strategies have been developed to circumnavigate this problem at a systems level. Parallel PA combination uses an array of switching PAs with different gains and sums their power together. Different amplifiers are switched on and off to obtain the desired amplitude variation at the output. ${ }^{6}$ PA out-phasing decompose the input into two constant 
envelope vectors and uses two switching PAs to amplify the signal then adds the power of the two back together. This vector addition process allows different amplitudes to be obtained as the angle between the two amplifiers is adjusted. ${ }^{7}$ Envelope following uses a DC-DC converter to modulate the supply of the PA and introduce the amplitude modulation. ${ }^{8}$ While using switching PAs in these approaches may allow the individual amplifiers to have high efficiency, the overall system efficiency is often significantly less than the amplifier. ${ }^{9}$ 


\section{Effect of Conduction Angle for Piecewise}

\section{Transfer Characteristic}

To simplify the analysis assume a piecewise transfer characteristic

$$
\text { for the transistor where } I_{d}= \begin{cases}0 & V_{i}<V_{O N} \\ g_{m}\left(V_{i}-V_{O N}\right) & V_{i}>V_{O N}\end{cases}
$$

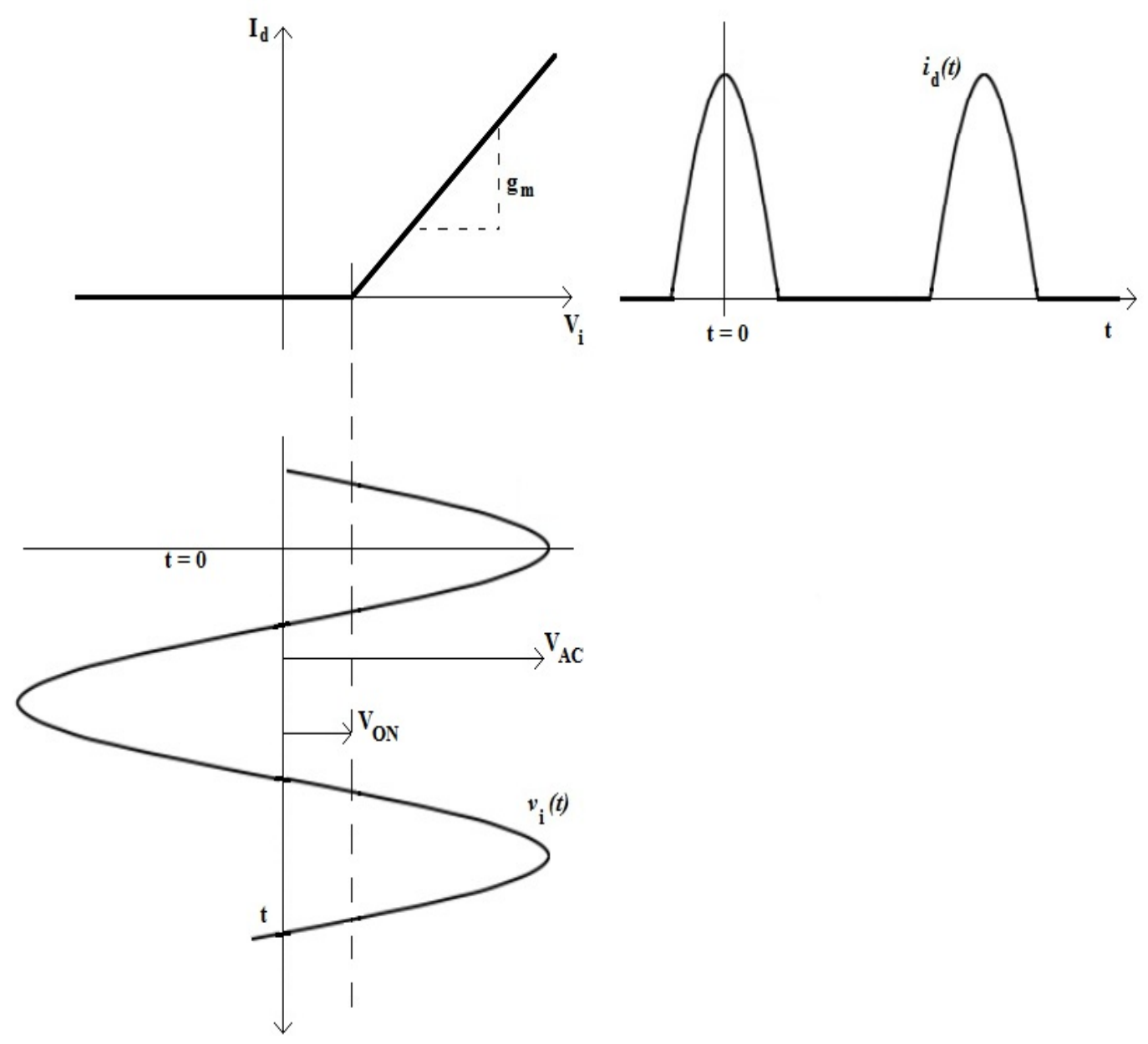

Figure 1: Voltage and current waveforms for piecewise approximation 
Expressing the conduction angle, $\theta$, in terms of $\mathrm{V}_{\mathrm{AC}}$ and $\mathrm{V}_{\mathrm{ON}}$

$$
\begin{gathered}
v_{i}=V_{A C} \cos \omega t \\
V_{O N}=v_{i} \text { when } \omega t=\frac{\theta}{2} \\
V_{O N}=V_{A C} \cos \frac{\theta}{2} \\
\theta=2 \cos ^{-1} \frac{V_{O N}}{V_{A C}}
\end{gathered}
$$

Examining the harmonic content of the drain current, by taking a fourier series of the expression

$$
i_{d}=g_{m}\left(v_{i}-V_{O N}\right)=g_{m}\left(V_{A C} \cos \omega t-V_{A C} \cos \frac{\theta}{2}\right)
$$

results in a DC term of

$$
\begin{aligned}
I_{D C} & =\frac{1}{T} \int_{T} i_{d} d t=\frac{1}{T} \int_{-\frac{\theta}{2 \omega}}^{\frac{\theta}{2 \omega}} g_{m}\left(V_{A C} \cos \omega t-V_{A C} \cos \frac{\theta}{2}\right) d t \\
= & \frac{\omega g_{m} V_{A C}}{\pi} \int_{0}^{\frac{\theta}{2 \omega}}\left(\cos \omega t-\cos \frac{\theta}{2}\right) d t=\left.\frac{\omega g_{m} V_{A C}}{\pi}\left(\frac{\sin \omega t}{\omega}-t \cos \frac{\theta}{2}\right)\right|_{0} ^{\frac{\theta}{2 \omega}} \\
= & \frac{\omega g_{m} V_{A C}}{\pi}\left(\frac{\sin \frac{\theta}{2}}{\omega}-\frac{\theta}{2 \omega} \cos \frac{\theta}{2}\right) \\
= & \frac{g_{m} V_{A C}}{\pi}\left(\sin \frac{\theta}{2}-\frac{\theta}{2} \cos \frac{\theta}{2}\right)
\end{aligned}
$$


and in $\mathrm{n}^{\text {th }}$ harmonic term of

$$
\begin{aligned}
& I_{n \omega}=\frac{2}{T} \int_{T} i_{d} \cos n \omega t d t=\frac{2}{T} \int_{-\frac{\theta}{2 \omega}}^{\frac{\theta}{2 \omega}} g_{m}\left(V_{A C} \cos \omega t-V_{A C} \cos \frac{\theta}{2}\right) \cos n \omega t d t \\
& =\frac{2 \omega g_{m} V_{A C}}{\pi} \int_{0}^{\frac{\theta}{2 \omega}}\left(\cos \omega t \cos n \omega t-\cos \frac{\theta}{2} \cos n \omega t\right) d t \\
& =\frac{2 \omega g_{m} V_{A C}}{\pi} \int_{0}^{\frac{\theta}{2 \omega}}\left(\frac{1}{2} \cos (n-1) \omega t+\frac{1}{2} \cos (n+1) \omega t-\cos \frac{\theta}{2} \cos n \omega t\right) d t \\
& =\frac{2 \omega g_{m} V_{A C}}{\pi}\left(\frac{1}{2(n-1) \omega} \sin (n-1) \omega t+\frac{1}{2(n+1) \omega} \sin (n+1) \omega t\right. \\
& \left.=\frac{1}{n \omega} \cos \frac{\theta}{2} \sin n \omega t\right)\left.\right|_{0} ^{\frac{\theta}{2 \omega}} \\
& =\frac{g_{m} V_{A C}}{\pi}\left(\frac{1}{(n-1)} \sin (n-1) \frac{\theta}{2}+\frac{1}{(n+1)} \sin (n+1) \frac{\theta}{2}-\frac{2}{n} \cos \frac{\theta}{2} \sin n \frac{\theta}{2}\right)
\end{aligned}
$$

For the fundamental frequency $n=1$

$$
I_{\omega}=\frac{g_{m} V_{A C}}{\pi}\left(\frac{\theta}{2}+\frac{1}{2} \sin \theta-2 \cos \frac{\theta}{2} \sin \frac{\theta}{2}\right)
$$

From this expression we can see that if $\theta$ is kept constant then $I_{\omega}$ is a linear function of $\mathrm{V}_{\mathrm{AC}}$. (1) shows that for $\theta$ to remain constant the ratio of $\mathrm{V}_{\mathrm{ON}}$ to $\mathrm{V}_{\mathrm{AC}}$ must remain constant. 
Note that when $\mathrm{V}_{\mathrm{ON}}=0 \mathrm{~V}$ then $\theta=\pi$ regardless of the value of $\mathrm{V}_{\mathrm{AC}}$, which is typical of class B operation.

Taking the ratio of the $\mathrm{DC}$ currents for a class $\mathrm{C}$ and class $\mathrm{B}$ amplifier we obtain

$$
\begin{gathered}
\frac{I_{D C}(\theta)}{I_{D C}(\pi)}=\frac{\frac{g_{m} V_{A C}}{\pi}\left(\sin \frac{\theta}{2}-\frac{\theta}{2} \cos \frac{\theta}{2}\right)}{\frac{g_{m} V_{A C}}{\pi}\left(\sin \frac{\pi}{2}-\frac{\pi}{2} \cos \frac{\pi}{2}\right)}=\frac{\sin \frac{\theta}{2}-\frac{\theta}{2} \cos \frac{\theta}{2}}{1} \\
=\sin \frac{\theta}{2}-\frac{\theta}{2} \cos \frac{\theta}{2}
\end{gathered}
$$

So by setting a fixed ratio for the DC current in each amplifier then the conduction angle of the class $\mathrm{C}$ amplifier can be set to a constant value.

By examining (3) and (5) we can also get a sense of how the conduction angle will affect the gain and efficiency of the amplifier.

From (5) we see that the gain for an amplifier, where output resistance is set so that $V_{\text {out }}=V_{D C}$ when $V_{\text {in }}=V_{D C}$, is

$$
\begin{gathered}
G=\frac{P_{\text {out }}}{P_{\text {in }}}=\frac{V_{\text {out }} I_{\text {out }}}{V_{\text {in }} I_{\text {in }}}=\frac{I_{\text {out }}}{I_{\text {in }}}=\frac{g_{m} V_{\text {in }}\left(\frac{\theta}{2 \pi}+\frac{1}{2 \pi} \sin \theta-\frac{2}{\pi} \cos \frac{\theta}{2} \sin \frac{\theta}{2}\right)}{\frac{V_{\text {in }}}{R_{\text {in }}}} \\
=g_{m} R_{\text {in }}\left(\frac{\theta}{2 \pi}+\frac{1}{2 \pi} \sin \theta-\frac{2}{\pi} \cos \frac{\theta}{2} \sin \frac{\theta}{2}\right)
\end{gathered}
$$




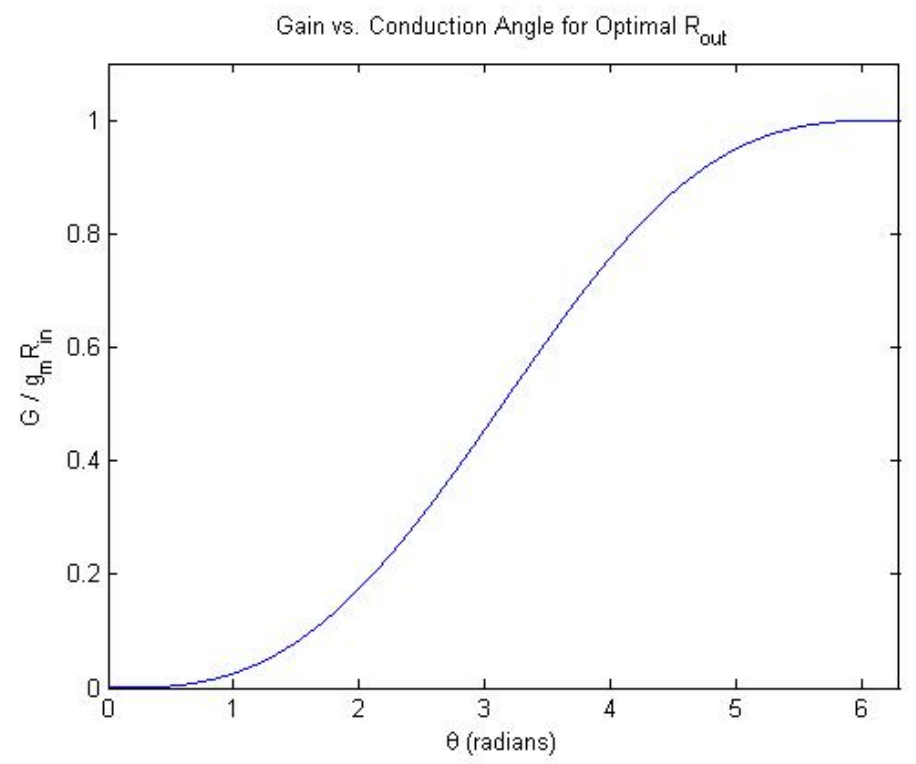

Figure 2: Gain as a function of conduction angle, $\mathbf{R}_{\text {out }}$ set for max swing

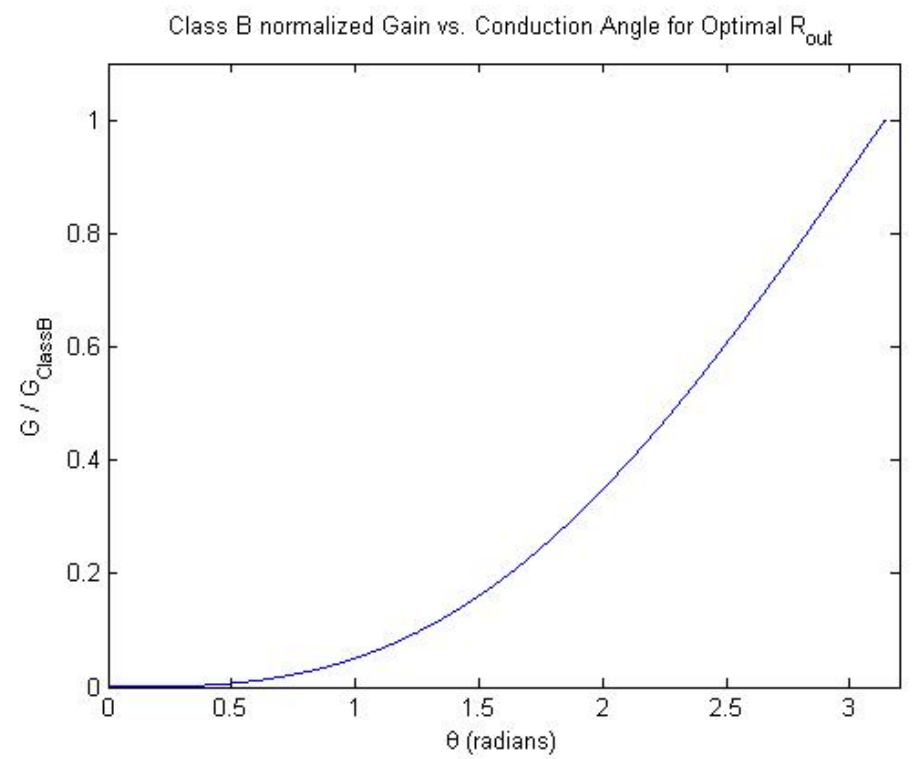

Figure 3: Gain relative to class B operation as a function of conduction angle, $\mathbf{R}_{\text {out }}$ set for max swing 


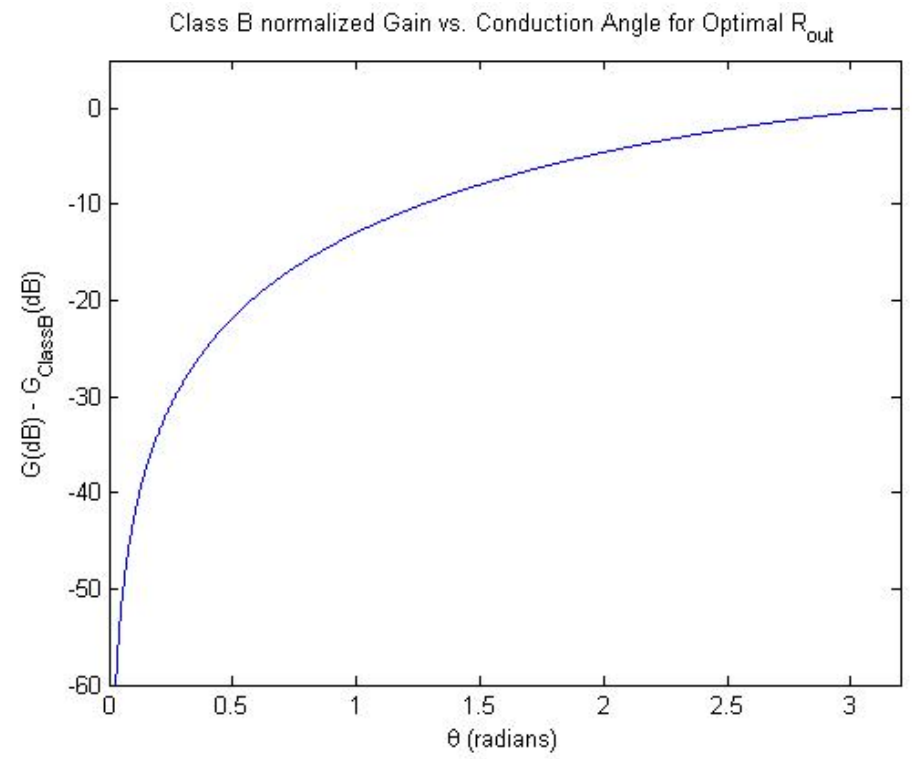

Figure 4: Gain relative to class $B$ operation, in $\mathrm{dB}$, as a function of conduction angle, $\mathbf{R}_{\text {out }}$ set for max swing

Figure 2 shows the relative gain of a linear amplifier as a function conduction angle, with $R_{\text {out }}$ set for max swing, all the way up to class $A$ bias. Figure 3 and Figure 4 show the relative gain, with $R_{\text {out }}$ set for max swing, up to a class $B$ bias.

The efficiency for such an amplifier is

$$
\begin{gathered}
\eta=\frac{P_{\text {out }}}{P_{D C}}=\frac{\frac{1}{2} V_{\text {out }} I_{\text {out }}}{V_{D C} I_{D C}}=\frac{\frac{1}{2} V_{\text {out }} \frac{g_{m} V_{A C}}{\pi}\left(\frac{\theta}{2}+\frac{1}{2} \sin \theta-2 \cos \frac{\theta}{2} \sin \frac{\theta}{2}\right)}{V_{\text {out }} \frac{g_{m} V_{A C}}{\pi}\left(\sin \frac{\theta}{2}-\frac{\theta}{2} \cos \frac{\theta}{2}\right)} \\
=\frac{\left(\frac{\theta}{2}+\frac{1}{2} \sin \theta-2 \cos \frac{\theta}{2} \sin \frac{\theta}{2}\right)}{2\left(\sin \frac{\theta}{2}-\frac{\theta}{2} \cos \frac{\theta}{2}\right)}
\end{gathered}
$$




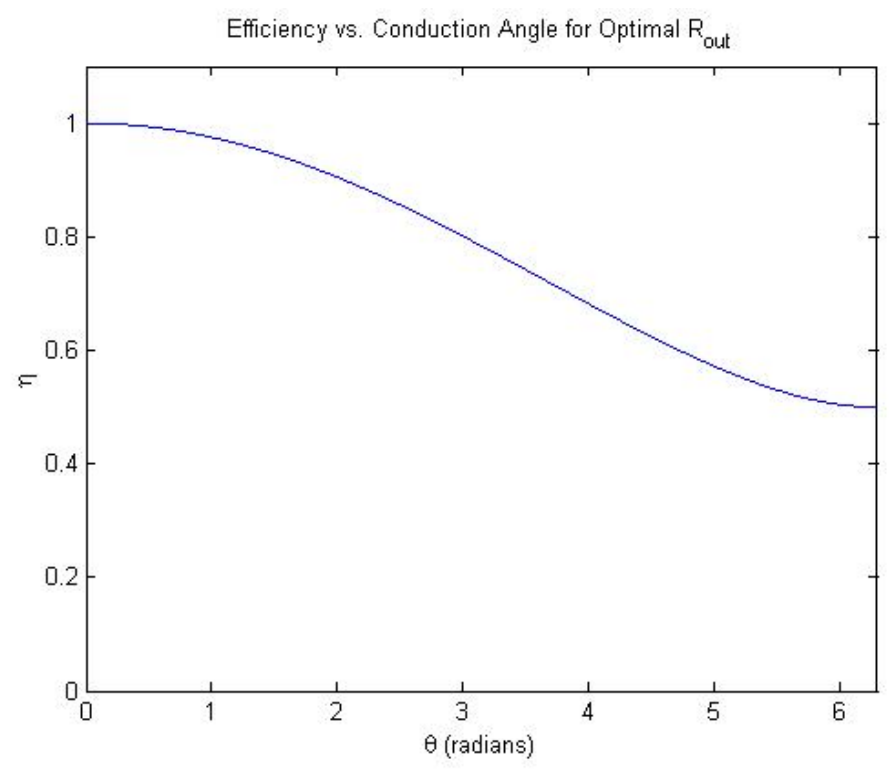

Figure 5: Efficiency as a function of conduction angle, $\mathbf{R}_{\text {out }}$ set for max swing

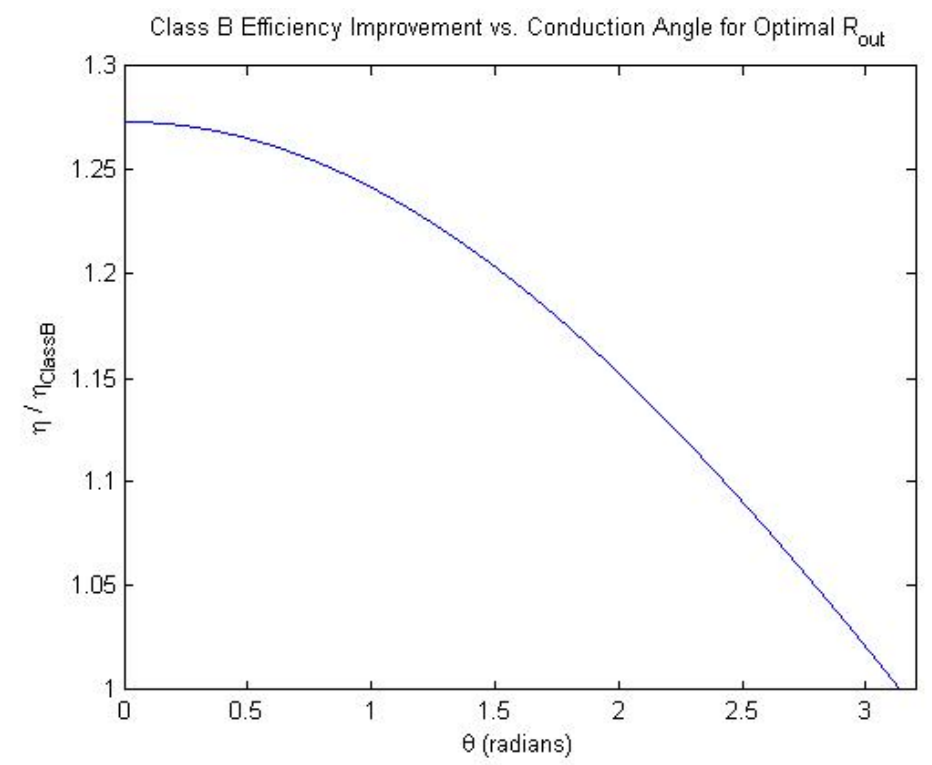

Figure 6: Efficiency relative to class B operation as a function of conduction angle, $\mathbf{R}_{\text {out }}$ set for max swing 
Figure 5 plots this efficiency as a function of conduction angle. This result agrees with the values cited in the literature of $50 \%$ efficiency at class $A, 78.5 \%$ efficiency at class $B$, and $100 \%$ efficiency at ideal class C. ${ }^{10}$ Figure 6 plots the relative improvement over class B efficiency as a function of conduction angle.

The gain for and amplifier where $V_{\text {in }}=V_{D C}$, but with fixed output resistance is (noting that since $V_{\text {in }}=V_{D C}, g_{m} R_{\text {out }}$ must be 1 or less for $V_{\text {out }}$ to remain below $\left.\mathrm{V}_{\mathrm{DC}}\right)$

$$
\begin{gathered}
G=\frac{P_{\text {out }}}{P_{\text {in }}}=\frac{I_{\text {out }}^{2} R_{\text {out }}}{\frac{V_{\text {in }}^{2}}{R_{\text {in }}}}=\frac{g_{m}^{2} V_{\text {in }}^{2}\left(\frac{\theta}{2 \pi}+\frac{1}{2 \pi} \sin \theta-\frac{2}{\pi} \cos \frac{\theta}{2} \sin \frac{\theta}{2}\right)^{2} R_{\text {out }} R_{\text {in }}}{V_{\text {in }}^{2}} \\
=g_{m}^{2}\left(\frac{\theta}{2 \pi}+\frac{1}{2 \pi} \sin \theta-\frac{2}{\pi} \cos \frac{\theta}{2} \sin \frac{\theta}{2}\right)^{2} R_{\text {out }} R_{\text {in }}
\end{gathered}
$$

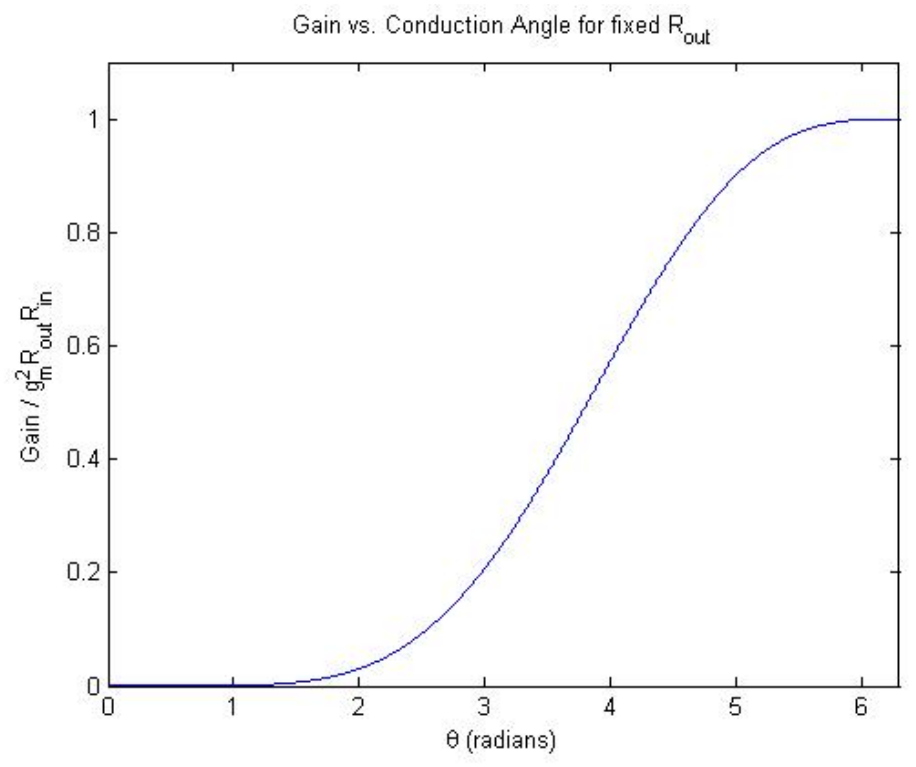

Figure 7: Gain as a function of conduction angle, $\mathbf{R}_{\text {out }}$ constant 


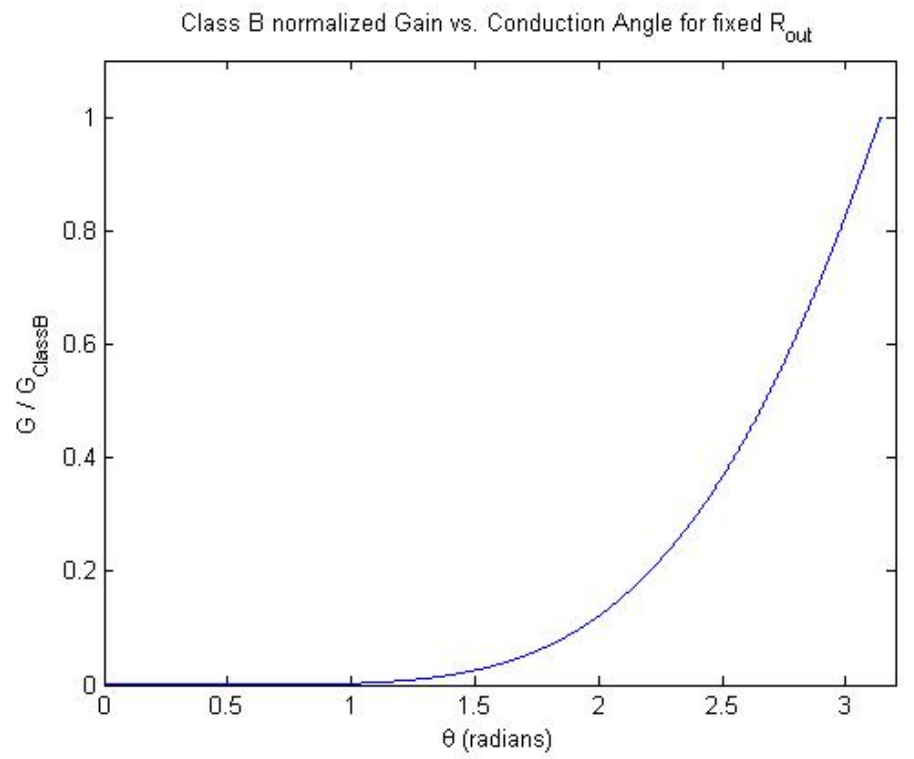

Figure 8: Gain relative to class $B$ operation as a function of conduction angle, $\mathbf{R}_{\text {out }}$ constant

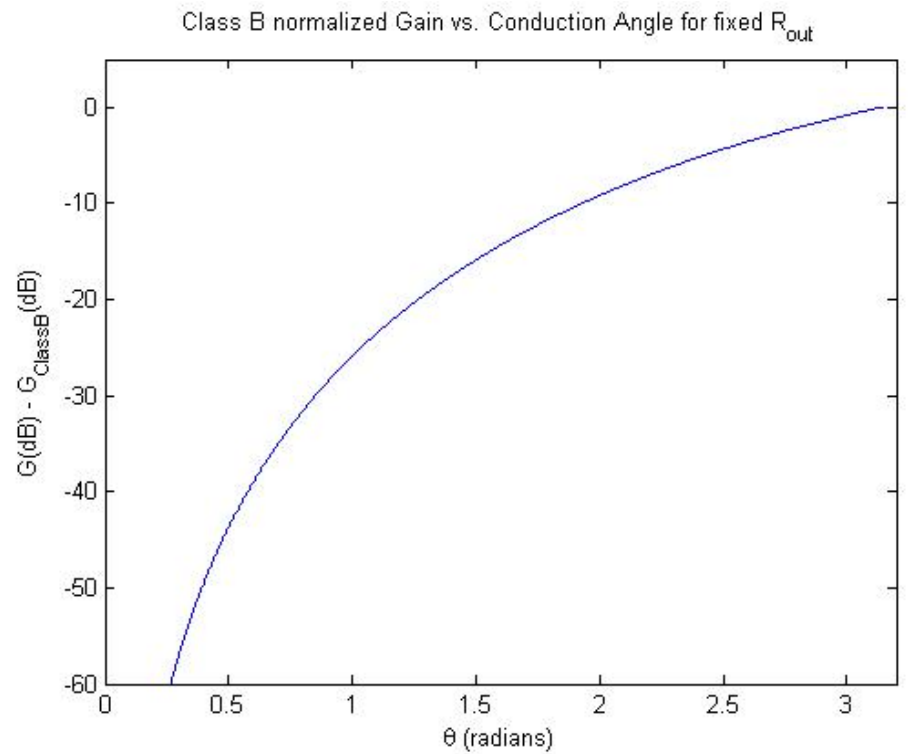

Figure 9: Gain relative to class B operation, in $\mathrm{dB}$, as a function of conduction angle, $\mathbf{R}_{\text {out }}$ constant 
Figure 7 shows the relative gain of a linear amplifier as a function conduction angle, with $R_{\text {out }}$ constant, all the way up to class $A$ bias. Figure 8 and Figure 9 show the relative gain, with $R_{\text {out }}$ constant, up to a class $B$ bias.

The efficiency for such an amplifier is

$$
\begin{gathered}
\eta=\frac{P_{\text {out }}}{P_{D C}}=\frac{\frac{1}{2} I_{\text {out }}^{2} R_{\text {out }}}{V_{D C} I_{D C}}=\frac{\frac{g_{m}^{2} V_{\text {in }}^{2}}{\pi^{2}}\left(\frac{\theta}{2}+\frac{1}{2} \sin \theta-2 \cos \frac{\theta}{2} \sin \frac{\theta}{2}\right)^{2} R_{\text {out }}}{2 V_{\text {in }} \frac{g_{m} V_{\text {in }}}{\pi}\left(\sin \frac{\theta}{2}-\frac{\theta}{2} \cos \frac{\theta}{2}\right)} \\
=\frac{\left(\frac{\theta}{2}+\frac{1}{2} \sin \theta-2 \cos \frac{\theta}{2} \sin \frac{\theta}{2}\right)^{2}}{2 \pi\left(\sin \frac{\theta}{2}-\frac{\theta}{2} \cos \frac{\theta}{2}\right)} g_{m} R_{\text {out }}
\end{gathered}
$$

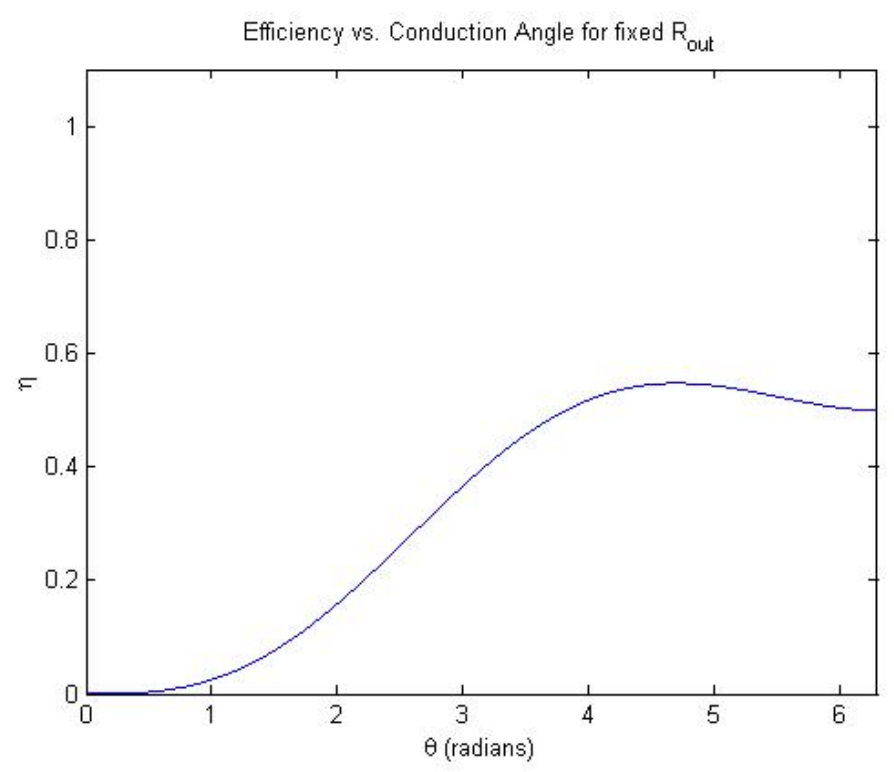

Figure 10: Efficiency as a function of conduction angle, $\mathbf{R}_{\text {out }}$ constant 


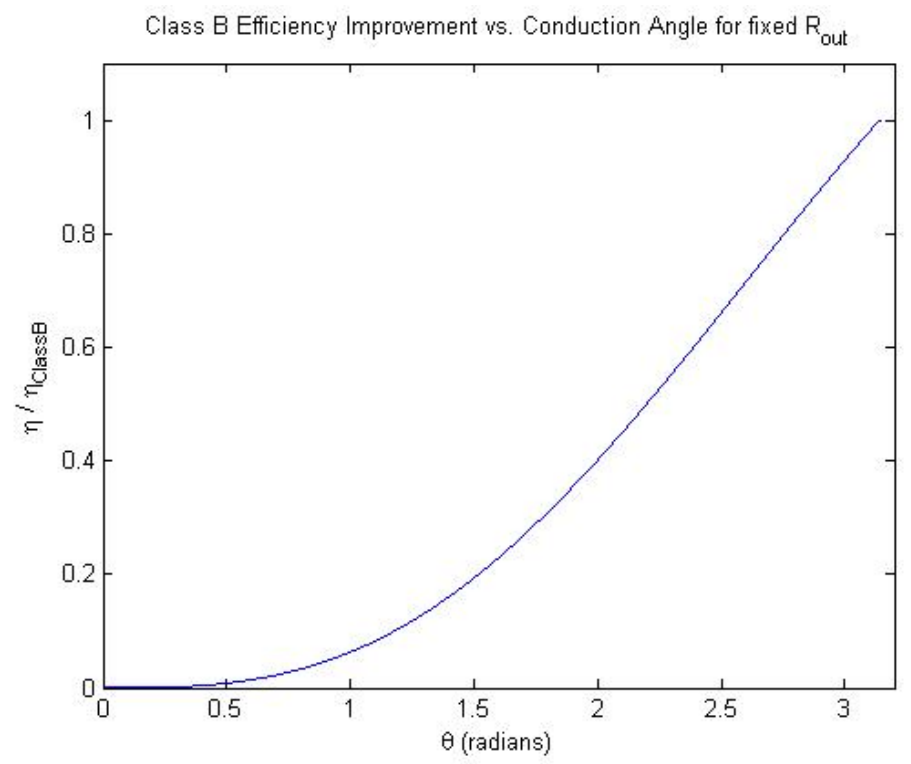

Figure 11: Efficiency relative to class $B$ operation as a function of conduction angle, $\mathbf{R}_{\text {out }}$ constant

From this analysis we can see that the load must be matched to obtain a full voltage swing at a given conduction angle, for decreasing the conduction angle to improve the efficiency. In other words, taking the same amplifier and just decreasing the conduction angle will actually hurt efficiency instead of improving it. 
Looking at the efficiency for a fixed conduction angle as a function of $V_{\text {in }}$

$$
\begin{gathered}
\eta=\frac{P_{\text {out }}}{P_{D C}}=\frac{\frac{1}{2} I_{\text {out }}^{2} R_{\text {out }}}{V_{D C} I_{D C}}=\frac{\frac{g_{m}^{2} V_{\text {in }}^{2}}{\pi^{2}}\left(\frac{\theta}{2}+\frac{1}{2} \sin \theta-2 \cos \frac{\theta}{2} \sin \frac{\theta}{2}\right)^{2} R_{\text {out }}}{2 V_{D C} \frac{g_{m} V_{\text {in }}}{\pi}\left(\sin \frac{\theta}{2}-\frac{\theta}{2} \cos \frac{\theta}{2}\right)} \\
c=\frac{\left(\frac{\theta}{2}+\frac{1}{2} \sin \theta-2 \cos \frac{\theta}{2} \sin \frac{\theta}{2}\right)^{2}}{\left(\sin \frac{\theta}{2}-\frac{\theta}{2} \cos \frac{\theta}{2}\right)} \\
\eta=c \frac{g_{m} R_{\text {out }}}{2 \pi V_{D C}} V_{\text {in }}
\end{gathered}
$$

So the efficiency will linearly decrease as the input voltage decreases. 
Since it is necessary to optimize $R_{\text {out }}$ for the desired angle, when designing an amplifier, the trade-off between gain and efficiency should determine the choice. Figure 12 shows gain relative to class $B$ operation and efficiency.

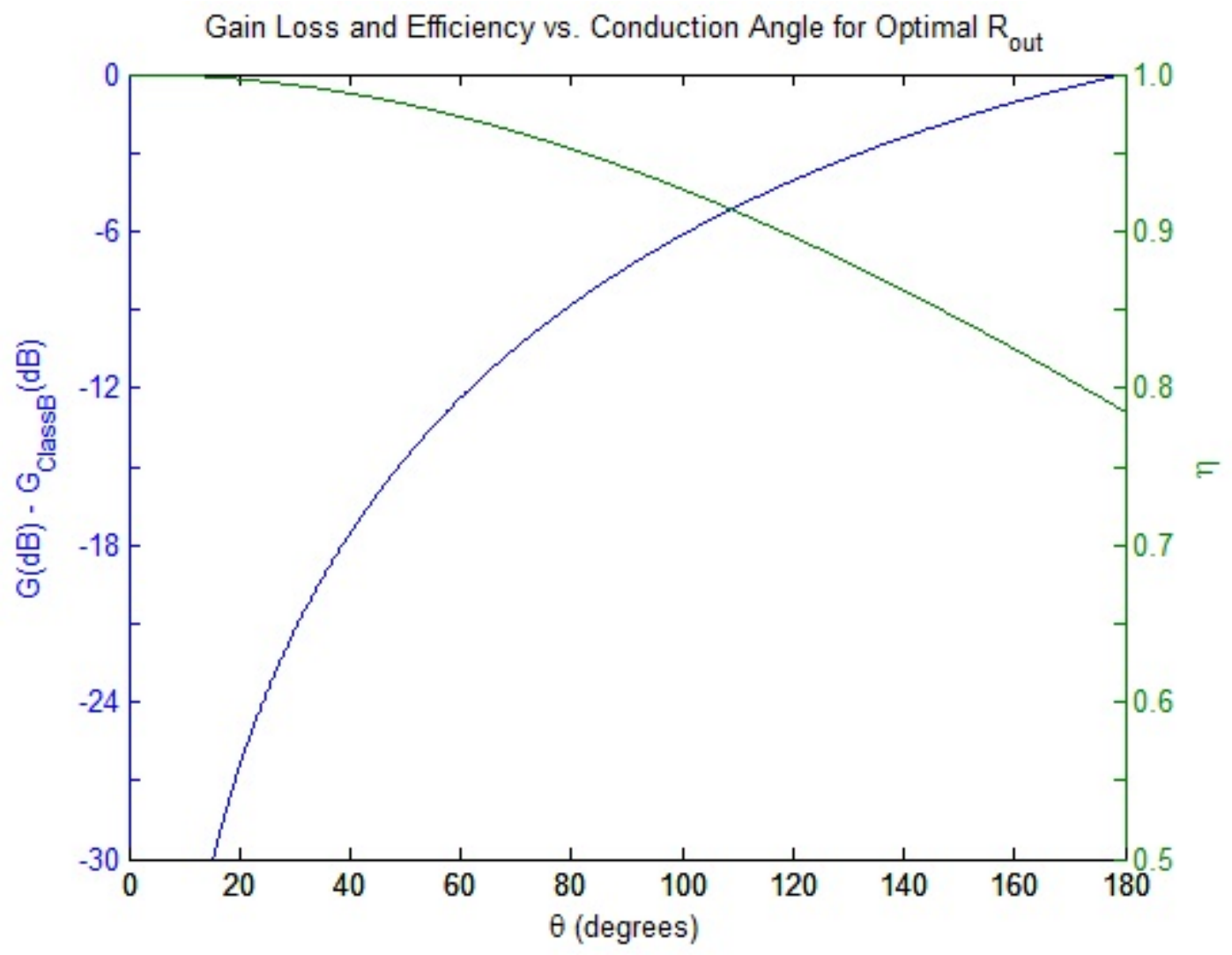

Figure 12: Gain-efficiency trade-off for Optimized $\mathbf{R}_{\text {out }}$ 


\section{CCAB Amplifier Implementation}

The system used in this research uses a transistor with a 500/1 width to length ratio as the actual amplifier. It then uses two matched transistors that have been scaled down to implement the biasing system.

Because close proximity results in similar processing and temperature conditions for all transistors, there should be minimal variation in performance from transistor to transistor. ${ }^{11}$

In this implementation one of the scaled down transistors receives a class B bias from a fixed voltage source. The other scaled down transistors shares its bias with the class C amplifier. From (6), if the DC current of the class $\mathrm{C}$ transistor is kept at a constant ratio to the DC current of the class B transistor, the conduction angle of the class $\mathrm{C}$ transistor should remain constant. To obtain this constant current ratio, the current of each transistor is mirrored to a lowpass transimpedance amplifier. This results in a voltage proportional to the DC current. These two voltages are input to a difference amplifier, which provides feedback to the bias as the input amplitude changes. By setting a different DC gain for each transimpedance amplifier the ratio of the two currents can be set, and therefore the desired conduction angle can be set.

Figure 13 shows a black box implementation to realize class $\mathrm{C}$ operation with constant conduction angle for changing input amplitude.

Figure 14 shows a circuit implementation to realize class $\mathrm{C}$ operation with constant conduction angle for changing input amplitude. 


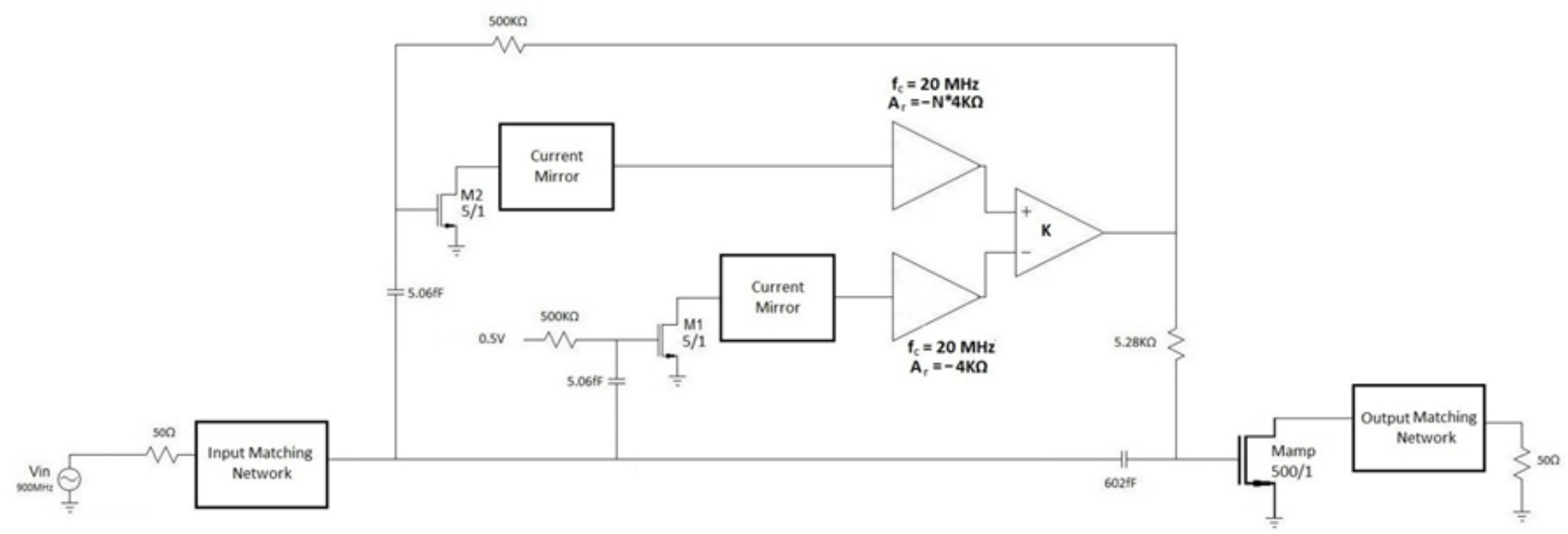

Figure 13: $C C A B$ amplifier diagram 


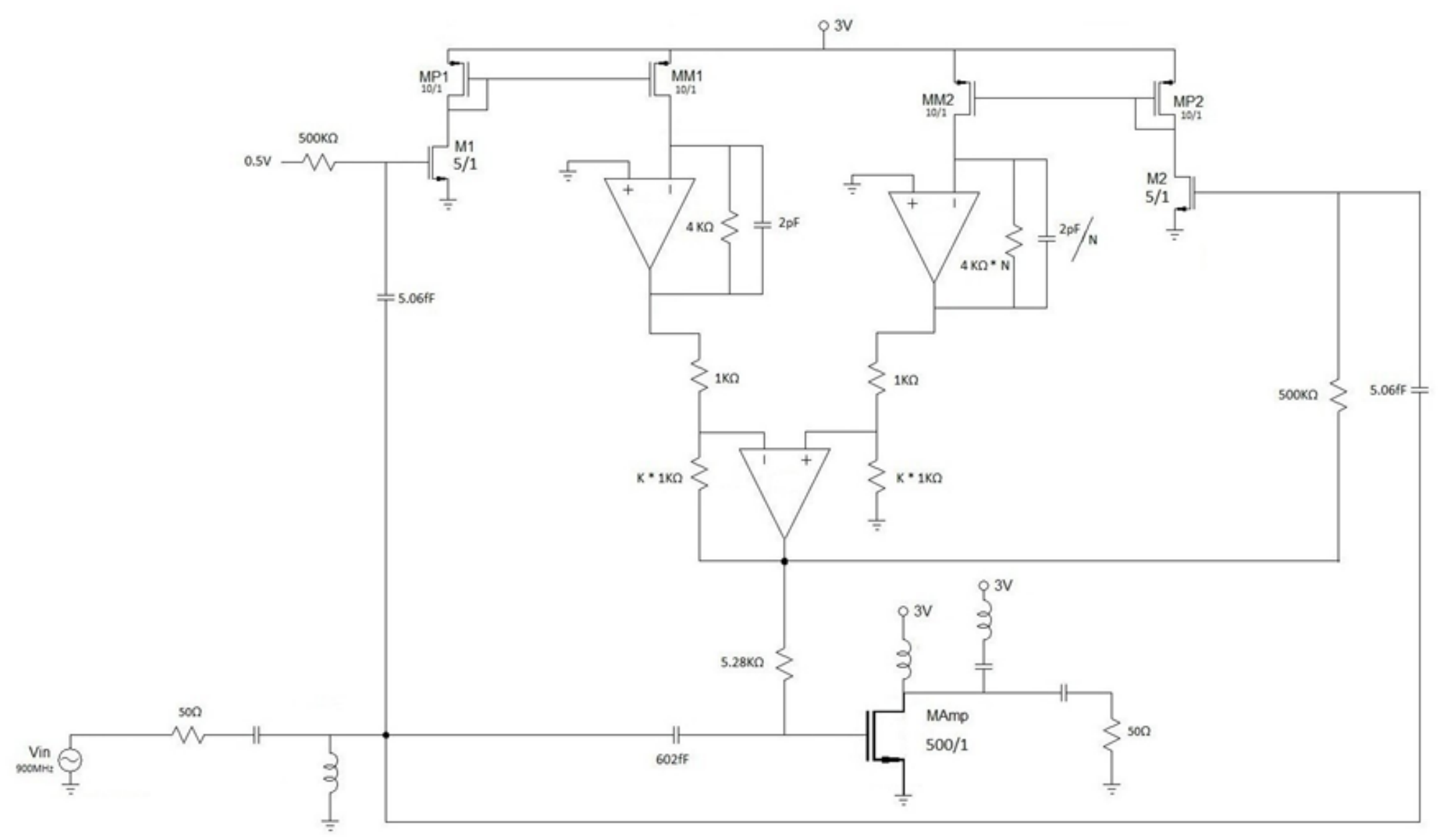

Figure 14: CCAB amplifier schematic 


\section{Design Process}

For the purpose of this research, the proposed CCAB biasing scheme will be used to examine the linearity at settings for several different conduction angles. Conduction angles of $\pi=180^{\circ}, 0.9 \pi=162^{\circ}$, $0.7 \pi=126^{\circ}, 0.5 \pi=90^{\circ}$, and $0.3 \pi=54^{\circ}$ will be examined. A class $B$ amplifier with a gain of $20 \mathrm{~dB}$ and $20 \mathrm{MHz}$ bandwidth at $900 \mathrm{MHz}$ will act as the launching point for the design. The class B amplifier will then have its gate bias adapted to produce several class $\mathrm{C}$ amplifiers, designed for the specified conduction angles occurring at $3 V_{p}$ input. The output matching networks for each of these amplifiers will also be adapted to give full output swing at $3 V_{p}$ input. These normally biased (NB) amplifiers will act as the control group for the research. The biasing for each of these amplifiers will then be implemented with the CCAB system. In the CCAB amplifiers, the resistors in the trans-impedance lowpass filter will be set for the $\mathrm{DC}$ current ratios that should ideally give the specified conduction angles. The gain of the difference amplifier will be tweaked for each conduction angle setting to produce a critically damped response for the bias. 


\section{Process Technology}

Transistor models were obtained from the T98b (7RF_6LM_ML) run of the MOSIS 180nm RF CMOS process. Level 49 spice models were used for both NMOS and PMOS transistors. These simulations were preformed in LTspice. The process supports 6 metal layers, MiM capacitors, and TaN metal level resistors. 


\section{Transistor Characterization}

For a MOSFET $R_{\text {in }}$ is much greater than $X_{i n}$, so the input resistance of the FET is negligible and the size of the resistor used to bias the transistor will greatly affect input resistance to the amplifier. Since the power drawn from the input source for any given voltage depends on the input resistance, the gain of the amplifier will depend on the size of the biasing resistor. This allows flexibility for sizing the transistor, since we can also change the size of the input resistor to obtain the desired gain. The transistors used in the biasing system should be small compared to the amplifying transistor. However, they should still be large enough to have a sharp turn on and not saturate at voltages significantly lower than the large amplifying transistor. A W/L ratio of $500 / 1$ for the transistor serving as the power amplifier and a W/L ratio of $5 / 1$ for the biasing transistors will satisfy the necessary criteria. 
Figure 15 and Figure 16 show the transfer characteristics for the $500 / 1$ and $5 / 1$ transistor respectively.

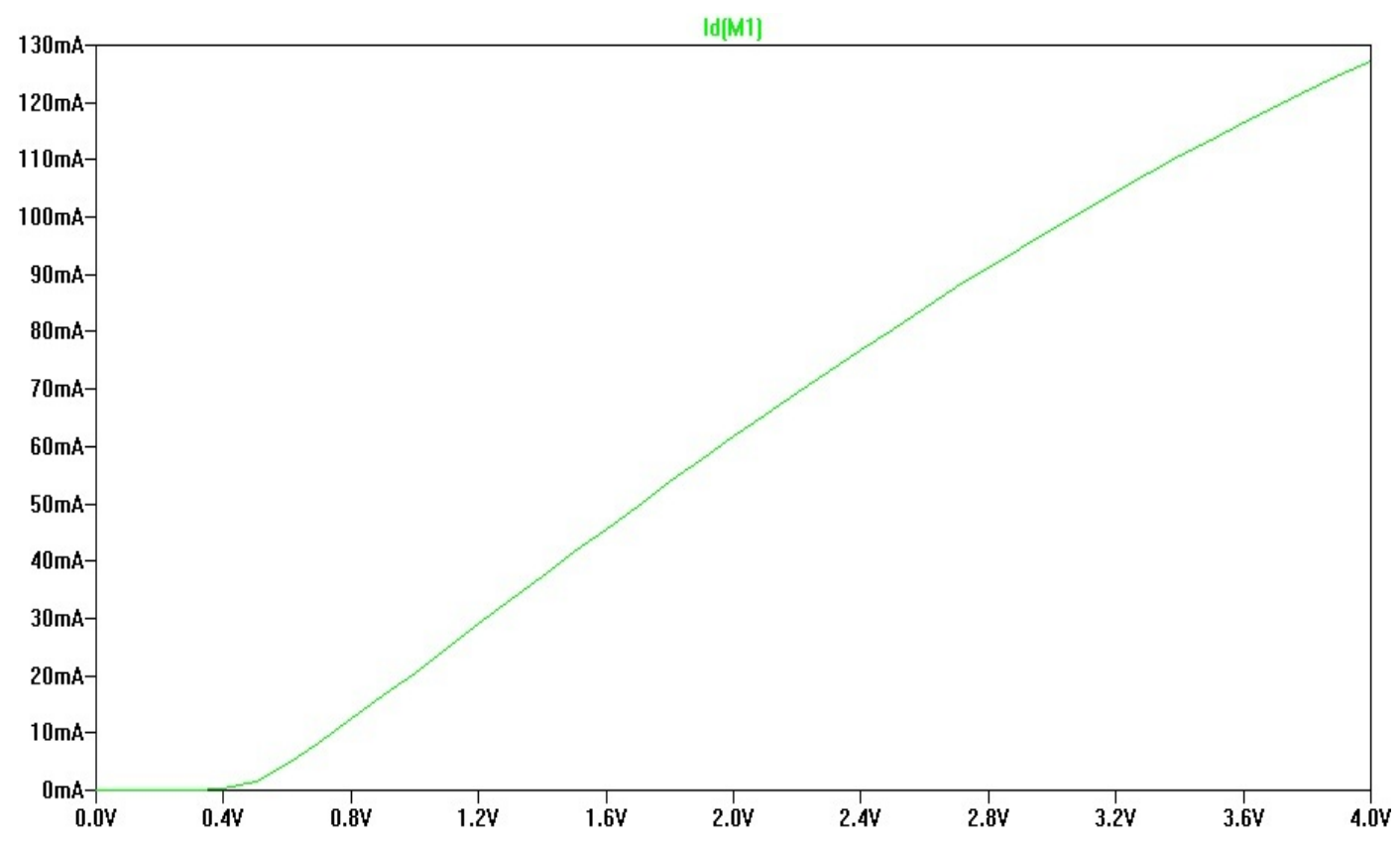

Figure 15: DC transfer characteristic for $W=90 \mu \mathrm{m} L=180 \mathrm{~nm}$

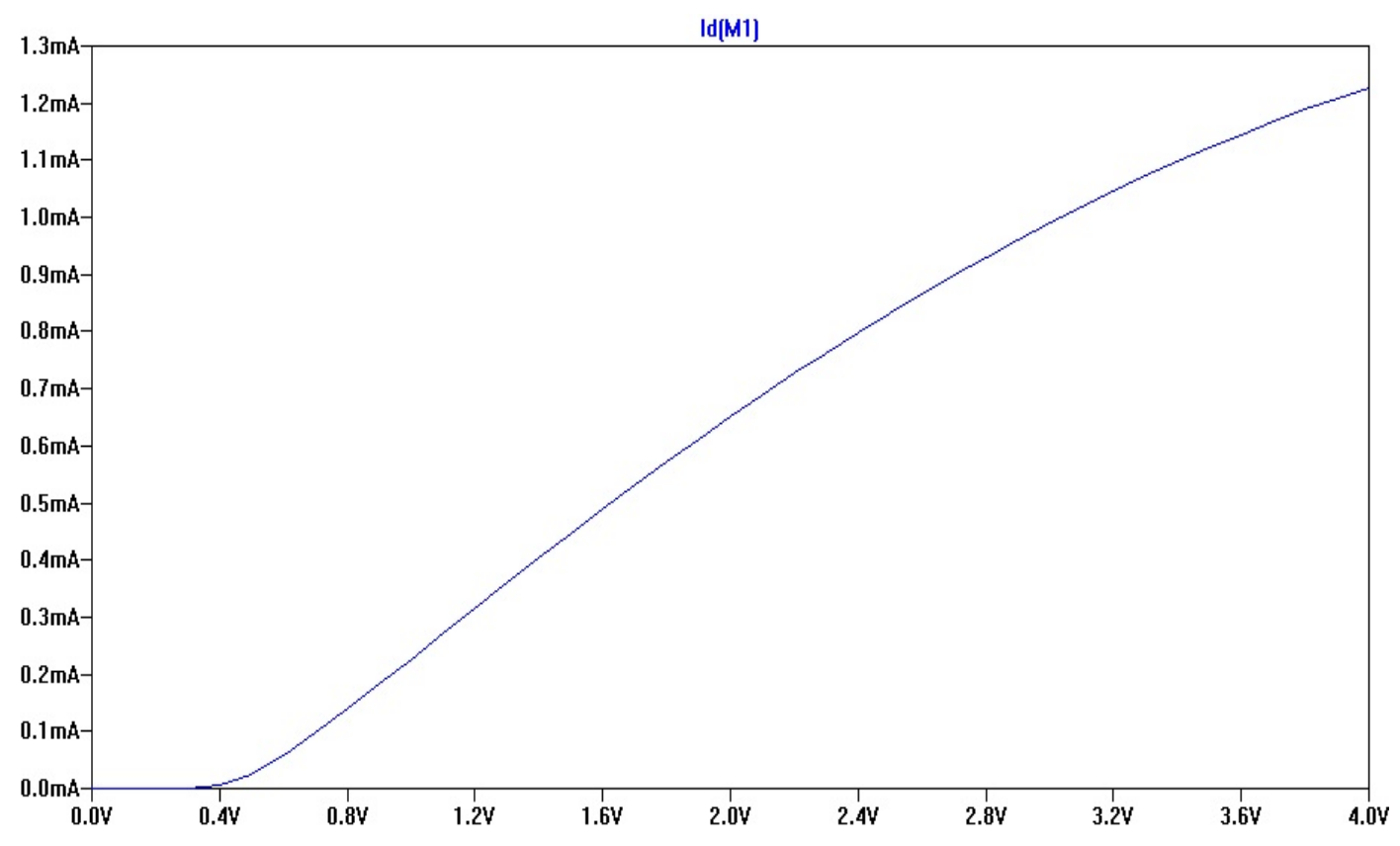

Figure 16: $D C$ transfer characteristic for $W=900 \mathrm{~nm} L=180 \mathrm{~nm}$ 
From the transfer characteristic we obtain $g_{m}$ by taking the slope between $0.8 \mathrm{~V}$ and $1.4 \mathrm{~V}$. Then we extract $\mathrm{V}_{\mathrm{t}}$ from $\mathrm{g}_{\mathrm{m}}$ and the value of the current at $0.8 \mathrm{~V}$. The current for a $3 \mathrm{~V}+\mathrm{V}_{\mathrm{t}}$, which is equivalent to a $3 \mathrm{~V}$ input for class B bias is obtained from the simulation and compared to the value for the ideal piecewise model. All of these values are shown in Table 1.

\begin{tabular}{|c|c|c|c|c|c|}
\hline $\mathrm{W} / \mathrm{L}$ & $\mathrm{g}_{\mathrm{m}}(\mathrm{mA} / \mathrm{V})$ & $\mathrm{V}_{\mathrm{t}}(\mathrm{V})$ & $\mathrm{I}_{\mathrm{d}} @\left(3 \mathrm{~V}+\mathrm{V}_{\mathrm{t}}\right)(\mathrm{mA})$ & $\mathrm{g}_{\mathrm{m}} \cdot 3 \mathrm{~V}(\mathrm{~mA})$ & $\Delta \mathrm{I}_{\mathrm{d}} @\left(3 \mathrm{~V}+\mathrm{V}_{\mathrm{t}}\right)$ \\
\hline $500 / 1$ & 41.6 & 0.5 & 113.6 & 124.8 & $-8.97 \%$ \\
\hline $5 / 1$ & 0.442 & 0.48 & 1.117 & 1.326 & $-15.76 \%$ \\
\hline
\end{tabular}

Table 1: Transfer parameters for $500 / 1$ and $5 / 1$ transistors

Figure 17 and Figure 18 show the output characteristic for the $500 / 1$ and $5 / 1$ transistor respectively. $\mathrm{V}_{\mathrm{g}}=0.5 \mathrm{~V} .1 .5 \mathrm{~V}$, and $3.5 \mathrm{~V}$.

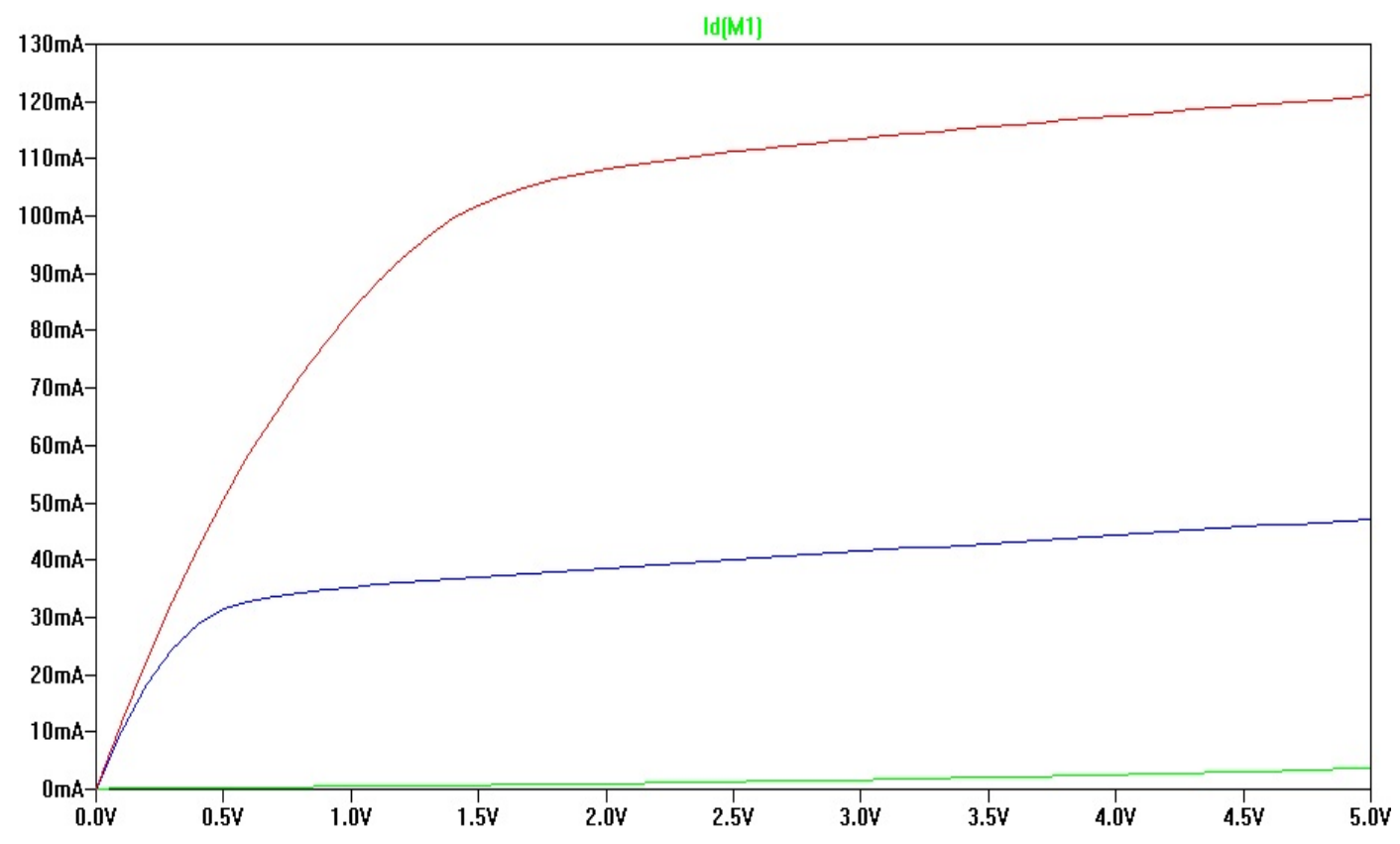

Figure 17: DC output characteristic for $W=90 \mu \mathrm{m} L=180 \mathrm{~nm}, V_{g}=0.5 \mathrm{~V}, 1.5 \mathrm{~V}, 3.5 \mathrm{~V}$ 


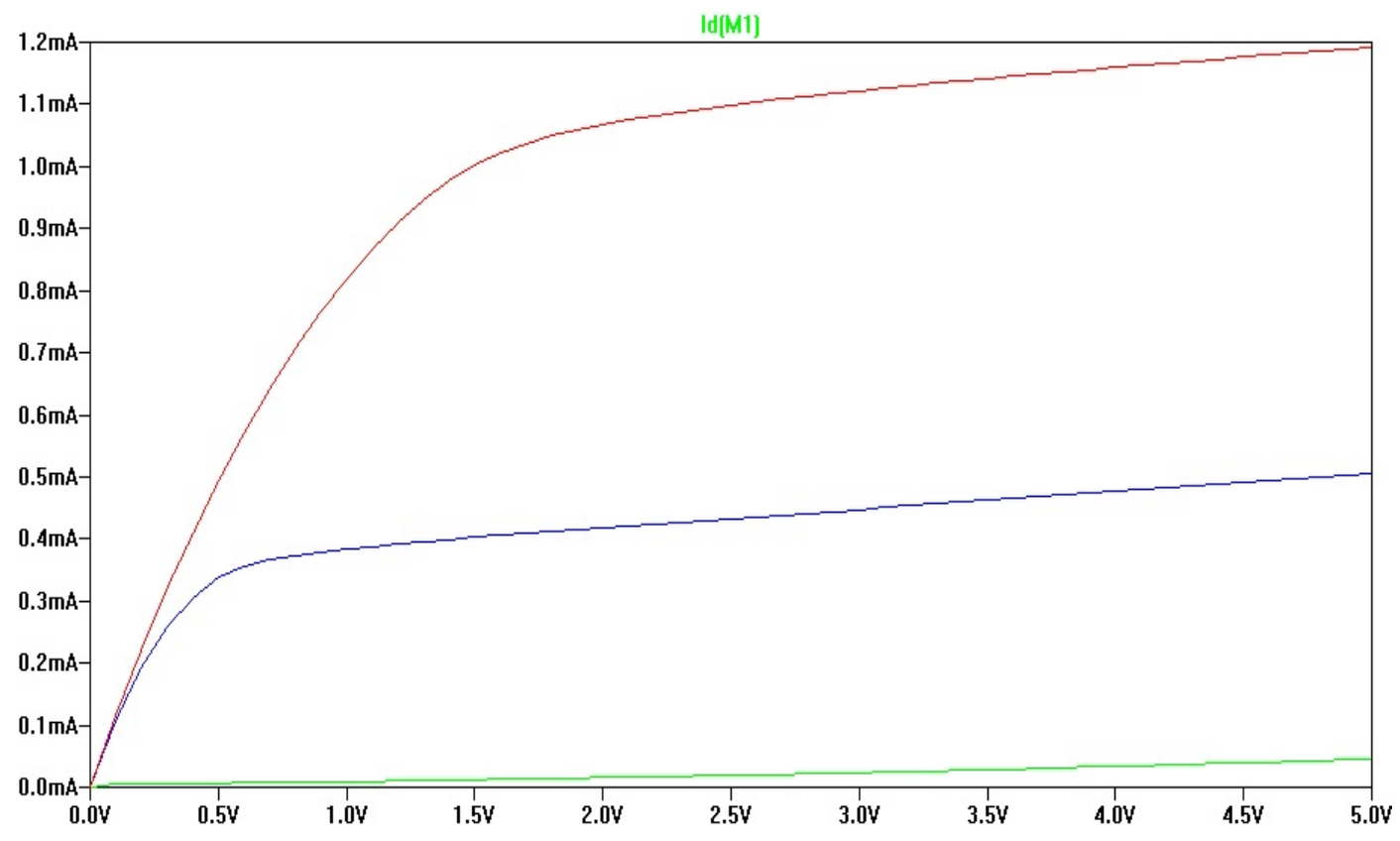

Figure 18: $D C$ output characteristic for $W=900 \mathrm{~nm} L=180 \mathrm{~nm}, V_{g}=0.5 \mathrm{~V}, 1.5 \mathrm{~V}, 3.5 \mathrm{~V}$

The amplifier is operating in a large signal capacity. Therefore, AC small signal analysis may not be suitable for finding the effective input impedance, since it will not consider the non linearity introduced by the transistor. So to measure the input impedance the transistor is biased at $\mathrm{V}_{\mathrm{g}}=0.5 \mathrm{~V}$ and $\mathrm{V}_{\mathrm{cc}}=3 \mathrm{~V}$. Then a $1 \mathrm{~V} 900 \mathrm{MHz}$ voltage is added to the gate. An FFT of the transient voltage and current are taken and the ratio of the two at $900 \mathrm{MHz}$ is the impedance. 


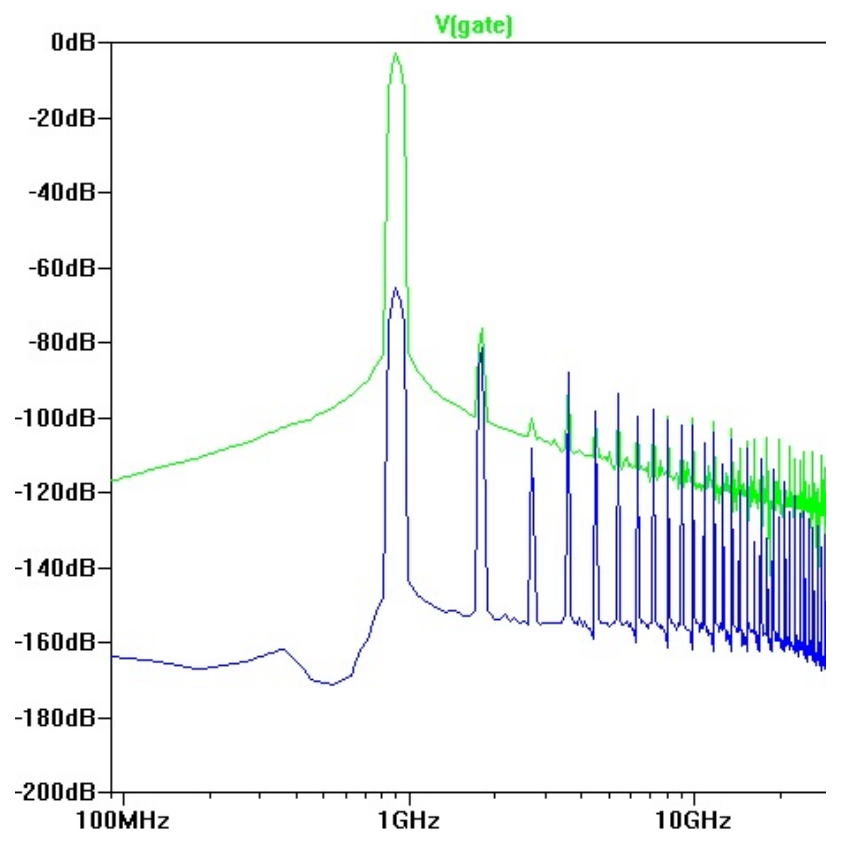

Figure 19: Gate voltage and current FFT for $W=90 \mu \mathrm{m} L=180 \mathrm{~nm}$

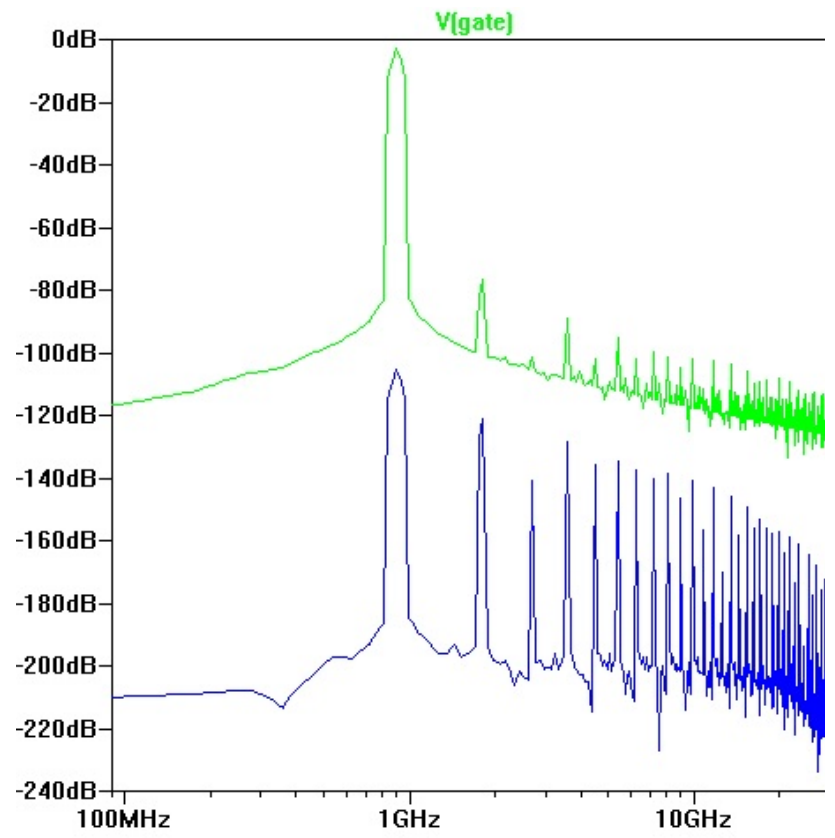

Figure 20: Gate voltage and current FFT for $\mathrm{W}=900 \mathrm{~nm} \mathrm{~L}=180 \mathrm{~nm}$ 


\begin{tabular}{|c|c|c|c|c|}
\hline $\mathrm{W} / \mathrm{L}$ & $\mathrm{V}_{\mathrm{g}}$ & $\mathrm{I}_{\mathrm{g}}$ & $\mathrm{Z}_{\text {in }}$ & $\mathrm{C}_{\text {in }}$ \\
\hline $500 / 1$ & $0.92 \mathrm{~V} \angle-90^{\circ}$ & $677 \mathrm{uA} \angle 0^{\circ}$ & $1.36 \mathrm{~K} \Omega \angle-90^{\circ}$ & $130 \mathrm{fF}$ \\
\hline $5 / 1$ & $0.89 \mathrm{~V} \angle-90^{\circ}$ & $7.1 \mathrm{uA} \angle 0^{\circ}$ & $125 \mathrm{~K} \Omega \angle-90^{\circ}$ & $1.41 \mathrm{fF}$ \\
\hline
\end{tabular}

Table 2: Input Impedance measurements

Even though an AC small signal analysis will not result in as accurate of a measurement, it should still be comparable to the result from the transient analysis. Using the .sweep and .measure command in conjunction with the $\mathrm{AC}$ analysis also allows us to analyze the how the input impedance will be affected by the gate bias.

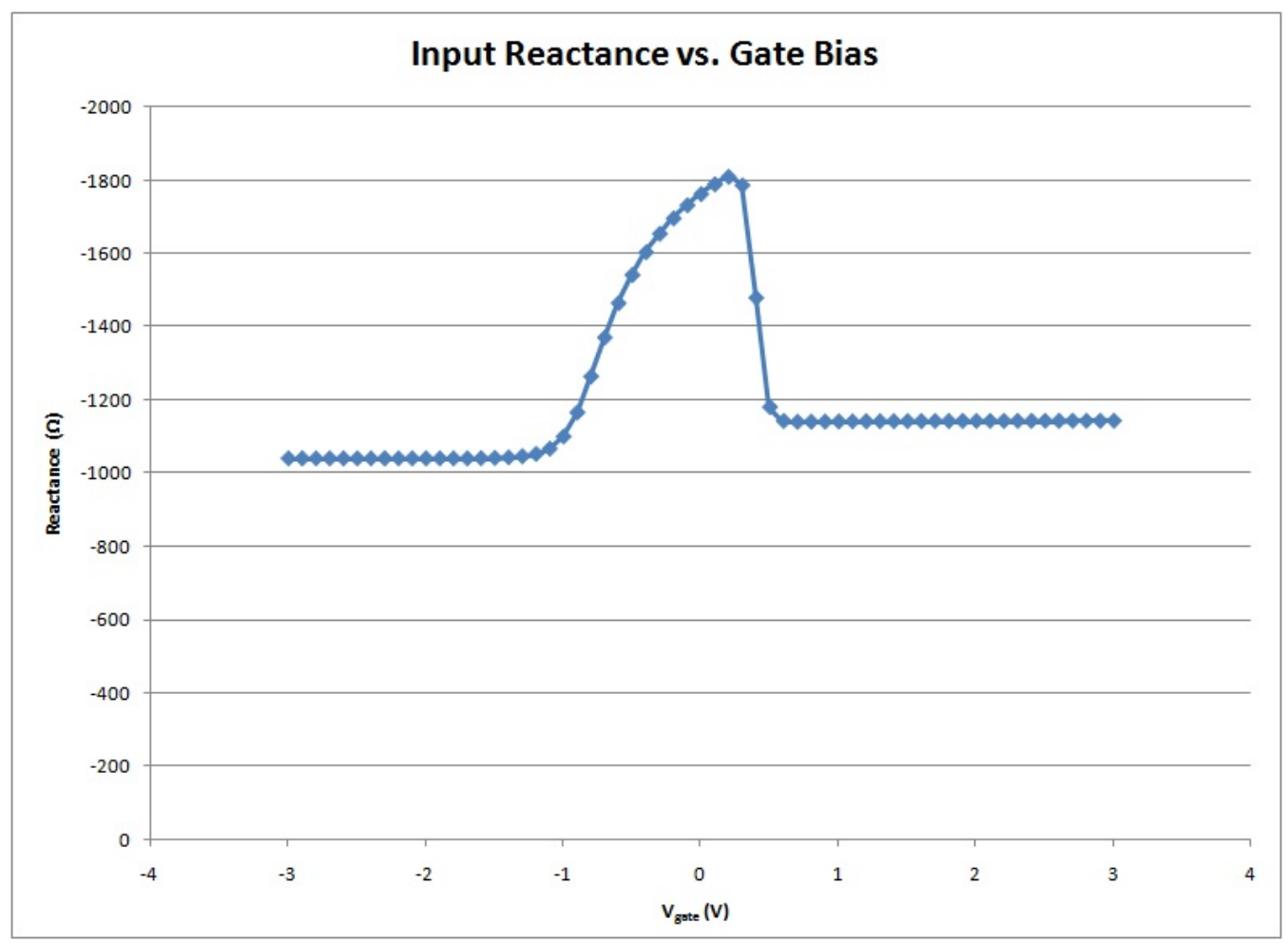

Figure 21: Small signal input reactance as a function of gate bias for $W=90 \mu m L=180 \mathrm{~nm}$ 


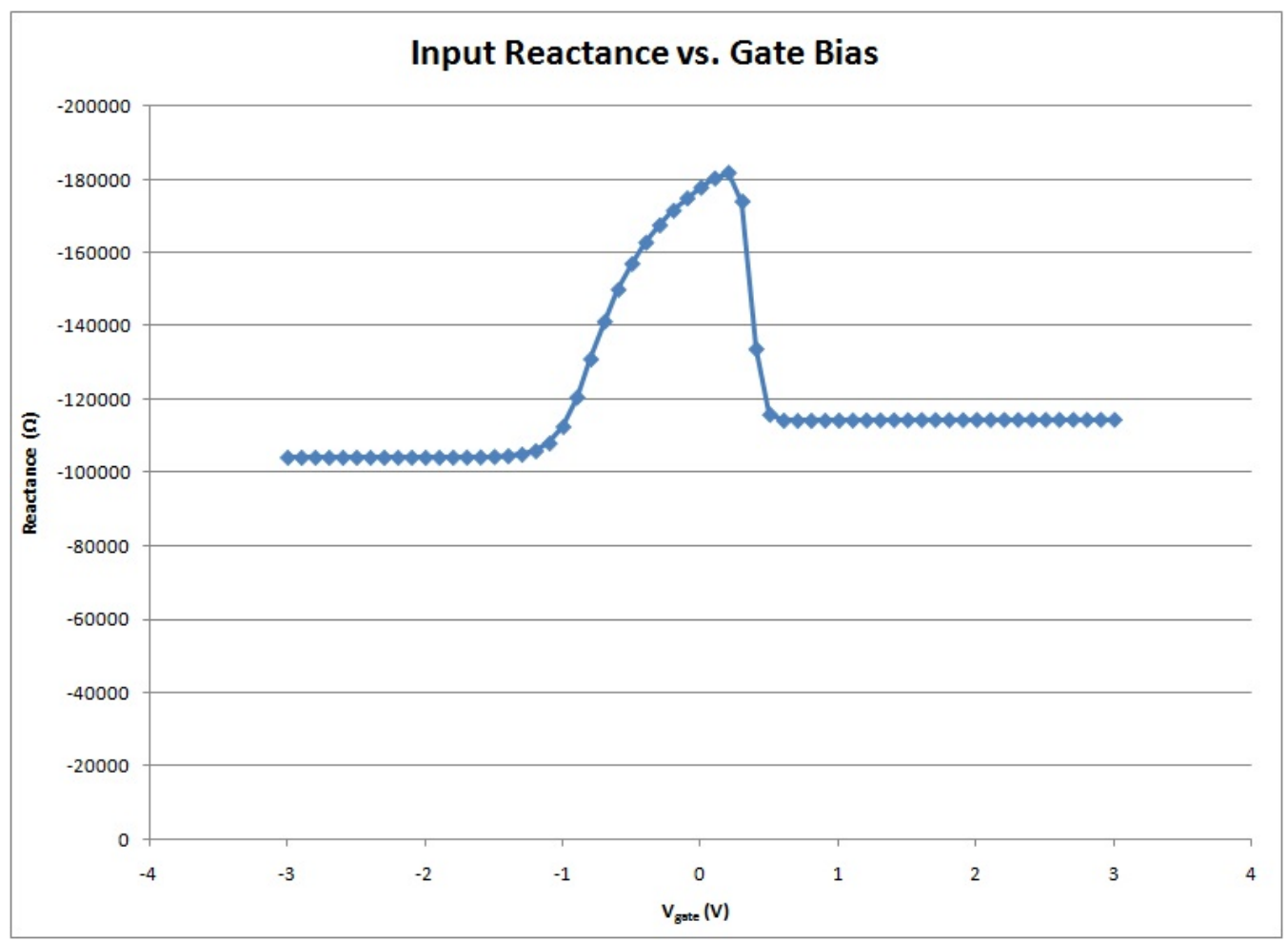

Figure 22: Small signal input reactance as a function of gate bias for $W=900 \mathrm{~nm} L=180 \mathrm{~nm}$

The effect of the non linear operation of the amplifier should not significantly affect the output impedance. Therefore we can analyze the output impedance with $\mathrm{AC}$ small signal analysis. 


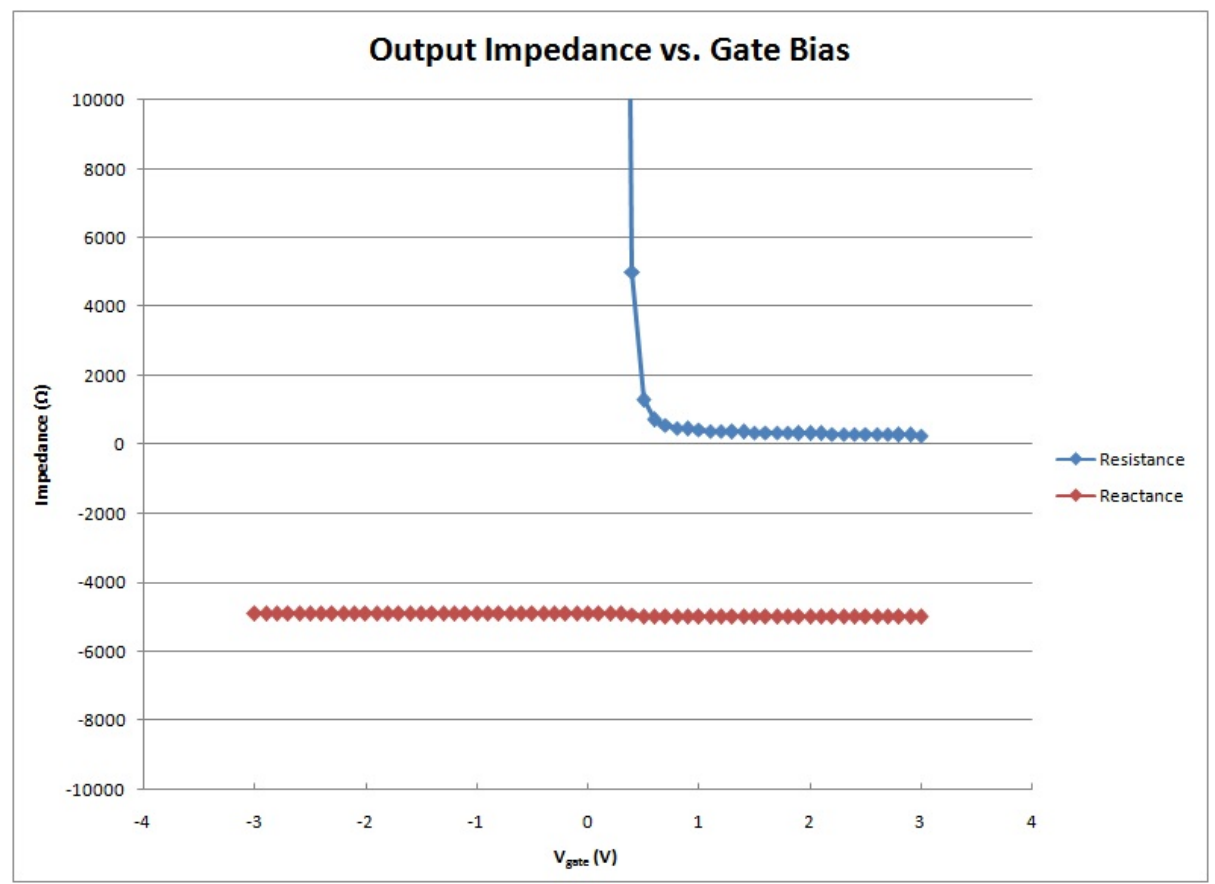

Figure 23: Small signal output impedance as a function of gate bias for $W=90 \mu \mathrm{m} L=180 \mathrm{~nm}$

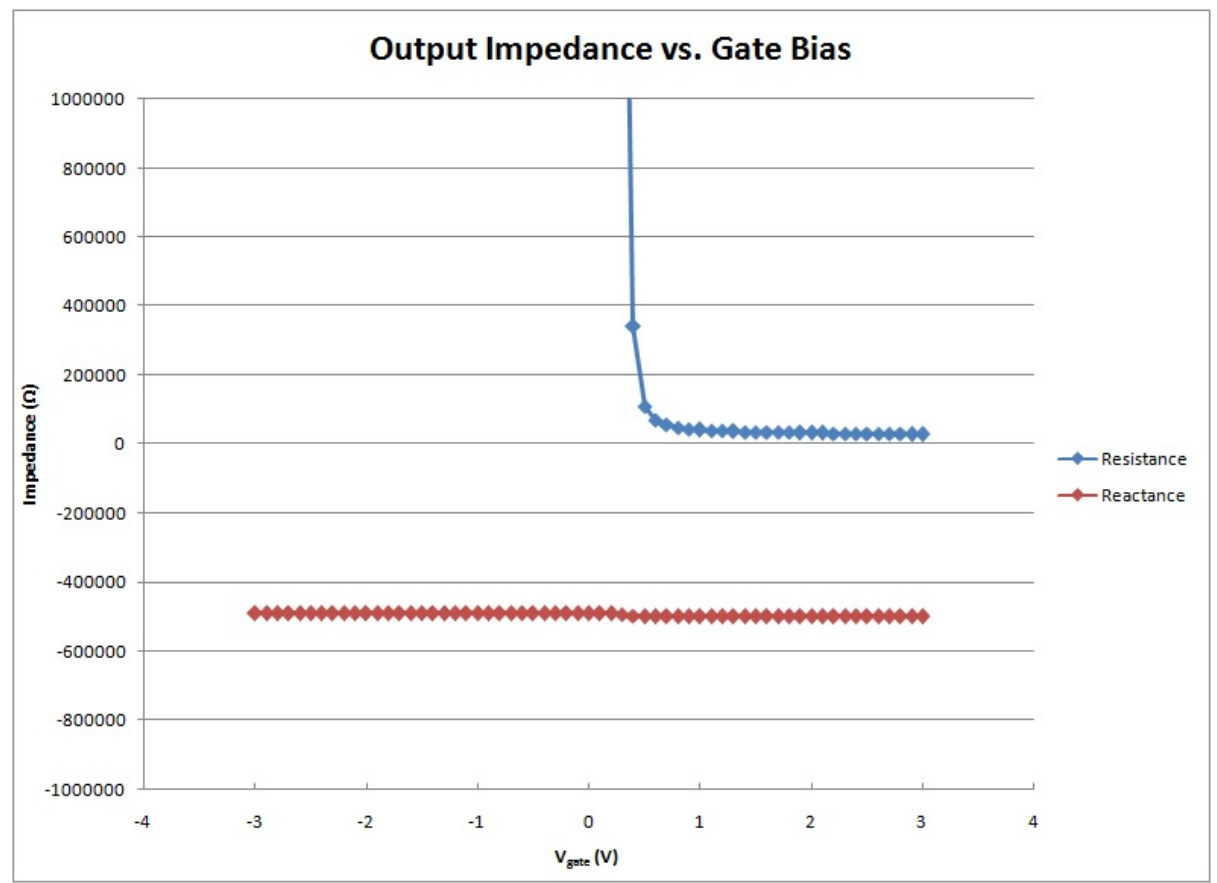

Figure 24: Small signal output impedance as a function of gate bias for $W=900 \mathrm{~nm} L=180 \mathrm{~nm}$ 
While the output resistance is finite for gate voltages above the turn on, it rapidly becomes negligible below the threshold.

For proper matching the goal is to have $3 \mathrm{~V}_{\mathrm{p}}$ at the output for the maximum current output. For class B operation of the 500/1 transistor the maximum current is $113.8 \mathrm{~mA}$. Substituting $\theta=\pi$ into (5) we obtain

$$
I_{o}=\frac{g_{m} V_{A C}}{2}=\frac{I_{\max }}{2}
$$

The desired output resistance is

$$
R=\frac{3 \mathrm{~V}}{I_{o}}=\frac{3 \mathrm{~V}}{\frac{113.8 \mathrm{~mA}}{2}}=52.7 \Omega
$$

Since the desired resistance is orders of magnitude smaller than the output resistance, the output resistance is negligible. As $\theta$ decreases, the value of $I_{0}$ decreases and the desired $R$ increases. But for smaller $\theta$ the gate bias will decrease and the output resistance will increase. So the output resistance should remain negligible compared to the desired $\mathrm{R}$ for all conduction angles.

\begin{tabular}{|c|c|c|c|c|c|c|}
\hline $\mathrm{W} / \mathrm{L}$ & $\mathrm{V}_{\mathrm{t}}$ & $\mathrm{g}_{\mathrm{m}}$ & $\mathrm{R}_{\text {in }}$ & $\mathrm{C}_{\text {in }}$ & $\mathrm{R}_{\text {out }}$ & $\mathrm{C}_{\text {out }}$ \\
\hline $500 / 1$ & 0.5 & 41.6 & $\infty$ & $130 \mathrm{fF}$ & $\infty$ & $36 \mathrm{fF}$ \\
\hline $5 / 1$ & 0.48 & 0.442 & $\infty$ & $1.41 \mathrm{fF}$ & $\infty$ & $.36 \mathrm{fF}$ \\
\hline
\end{tabular}

Table 3: Summary of transistor characteristics 


\section{Input Coupling and Biasing}

Figure 25 shows the set up for the input to each transistor.

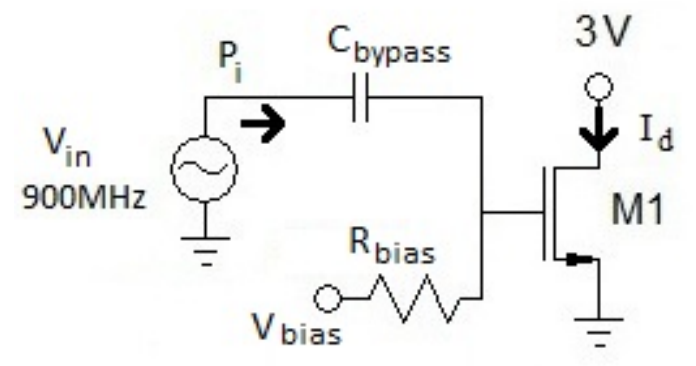

Figure 25: Input biasing network

A gain of $20 \mathrm{~dB}$ is the target for class $B$ operation of the $500 / 1$

transistor. At the maximum input of approximately $3 V_{p}$, we want our load to result in drain voltage also having a $3 \mathrm{~V}_{p}$ swing.

$$
G=20 \mathrm{~dB}=100=\frac{P_{o}}{P_{i}}=\frac{\frac{1}{2} \operatorname{Re}\left(V_{o} I_{o}^{*}\right)}{P_{i}}
$$

For a properly matched load $V_{o}$ and $I_{0}$ will be in phase.

$$
\begin{gathered}
G=\frac{\frac{1}{2}\left|V_{o}\right|\left|I_{o}\right|}{P_{i}} \\
I_{o}=\frac{2 G P_{i}}{3 V}
\end{gathered}
$$

Substituting in (12) 


$$
I_{\max }=\frac{4 G P_{i}}{3 V}
$$

Then

$$
\begin{gathered}
P_{i}=\frac{\left|V_{g}\right|^{2}}{R_{\text {in }} \| R_{\text {bias }}} \\
R_{\text {in }} \approx \infty \\
P_{i}=\frac{1}{2} \frac{\left|V_{g}\right|^{2}}{R_{\text {bias }}}
\end{gathered}
$$

Substituting into (13)

$$
\begin{gathered}
I_{\text {max }}=\frac{2 G \cdot 3 V}{R_{\text {bias }}}=113.6 \mathrm{~mA} \\
R_{\text {bias }}=5.28 \mathrm{~K} \Omega
\end{gathered}
$$

The RC pole acts as a low pass filter to the feedback system and must be placed above the bandwidth

$$
\begin{gathered}
\frac{1}{R_{\text {bias }}\left(C_{\text {bypass }}+C_{\text {in }}\right)}=n \cdot 2 \pi f_{b}=n \cdot 2 \pi f_{o} \frac{2 \pi f_{b}}{2 \pi f_{o}}=\frac{n \omega_{o}}{Q} \\
\omega C_{\text {bypass }}=\frac{Q}{n R_{\text {bias }}}-\omega C_{\text {in }}
\end{gathered}
$$


Since the input impedance for the $5 / 1$ transistors will be larger than the $500 / 1$ transistor, we would like $\left|V_{g}\right| /\left|V_{\text {in }}\right|$ to be as close to 1 as possible.

$$
\begin{aligned}
& \left|\frac{V_{g}}{V_{\text {in }}}\right|=\left|\frac{R_{\text {bias }} \|-\frac{j}{\omega C_{\text {in }}}}{\left(R_{\text {bias }} \|-\frac{j}{\omega C_{\text {in }}}\right)-\frac{j}{\omega C_{\text {bypass }}}}\right|=\left|\frac{j \omega C_{\text {bypass }} R_{\text {bias }}}{1+j \omega R_{\text {bias }}\left(C_{\text {in }}+C_{\text {bypass }}\right)}\right| \\
& =\left|\frac{j \omega C_{\text {bypass }} R_{\text {bias }}}{1+j \omega \frac{Q}{n \omega}}\right|=\frac{\omega C_{\text {bypass }} R_{\text {bias }}}{\sqrt{1+\left(\frac{Q}{n}\right)^{2}}} \\
& =\frac{\frac{Q}{n}-\omega C_{\text {in }} R_{\text {bias }}}{\sqrt{1+\left(\frac{Q}{n}\right)^{2}}}=\frac{\frac{45}{n}-3.88}{\sqrt{1+\left(\frac{45}{n}\right)^{2}}}
\end{aligned}
$$

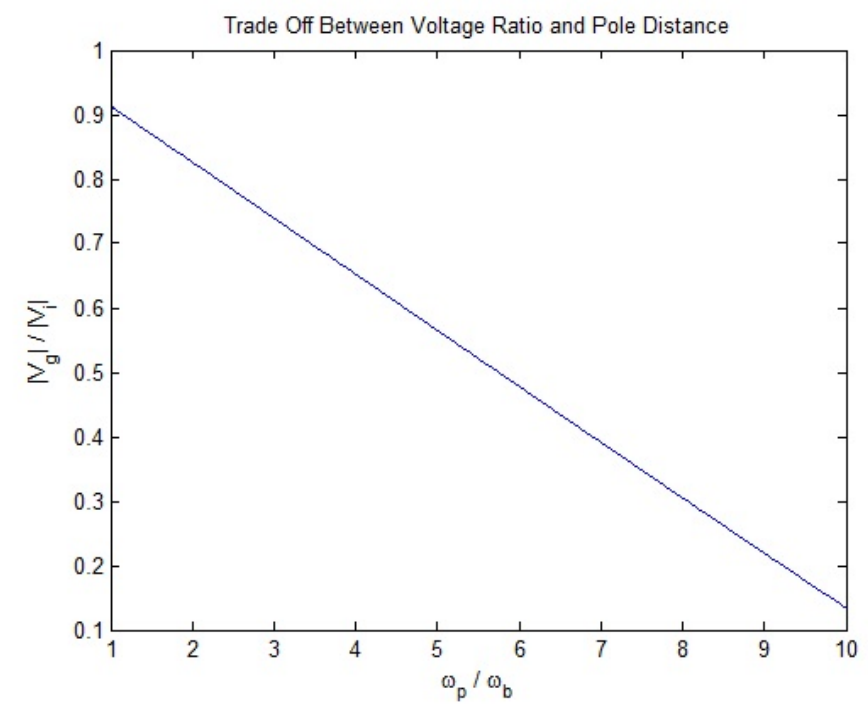

Figure 26: Voltage divider ratio as a function of pole to bandwidth ratio 
At $\mathrm{n}=2.5$ a divider ratio of 0.78 should be acceptable.

$$
\begin{gathered}
C_{\text {bypass }}=\frac{Q}{n \omega R_{\text {bias }}}-C_{\text {in }}=602 \mathrm{fF} \\
V_{\text {in }}=\frac{V_{g}}{0.78}=3.83 \mathrm{~V}
\end{gathered}
$$

Simulating the 500/1 transistor the following wave forms were obtained

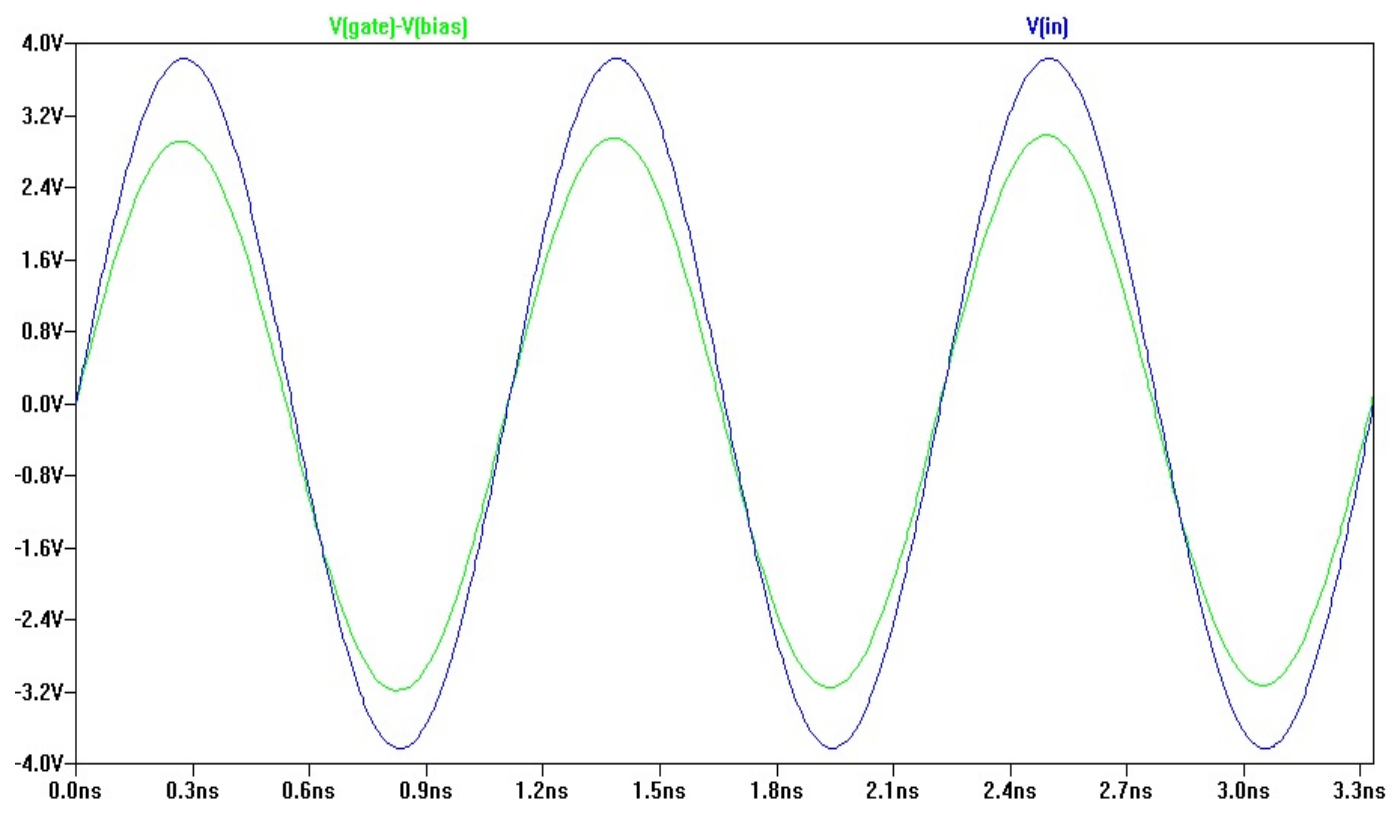

Figure 27: Input voltage and AC gate voltage for $W=90 \mu \mathrm{m} L=180 \mathrm{~nm}$ 


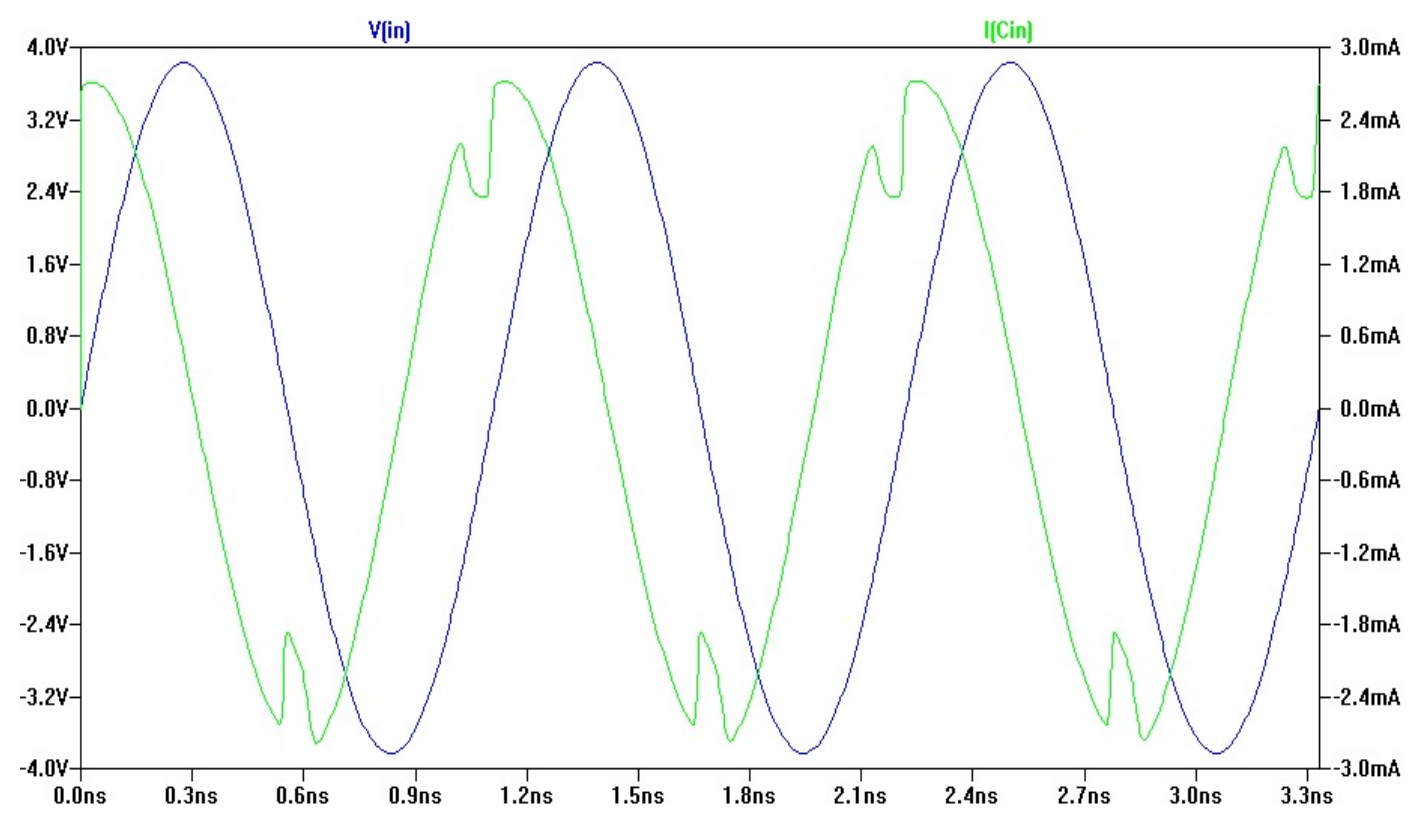

Figure 28: Input voltage and input current for $W=90 \mu \mathrm{m} L=180 \mathrm{~nm}$

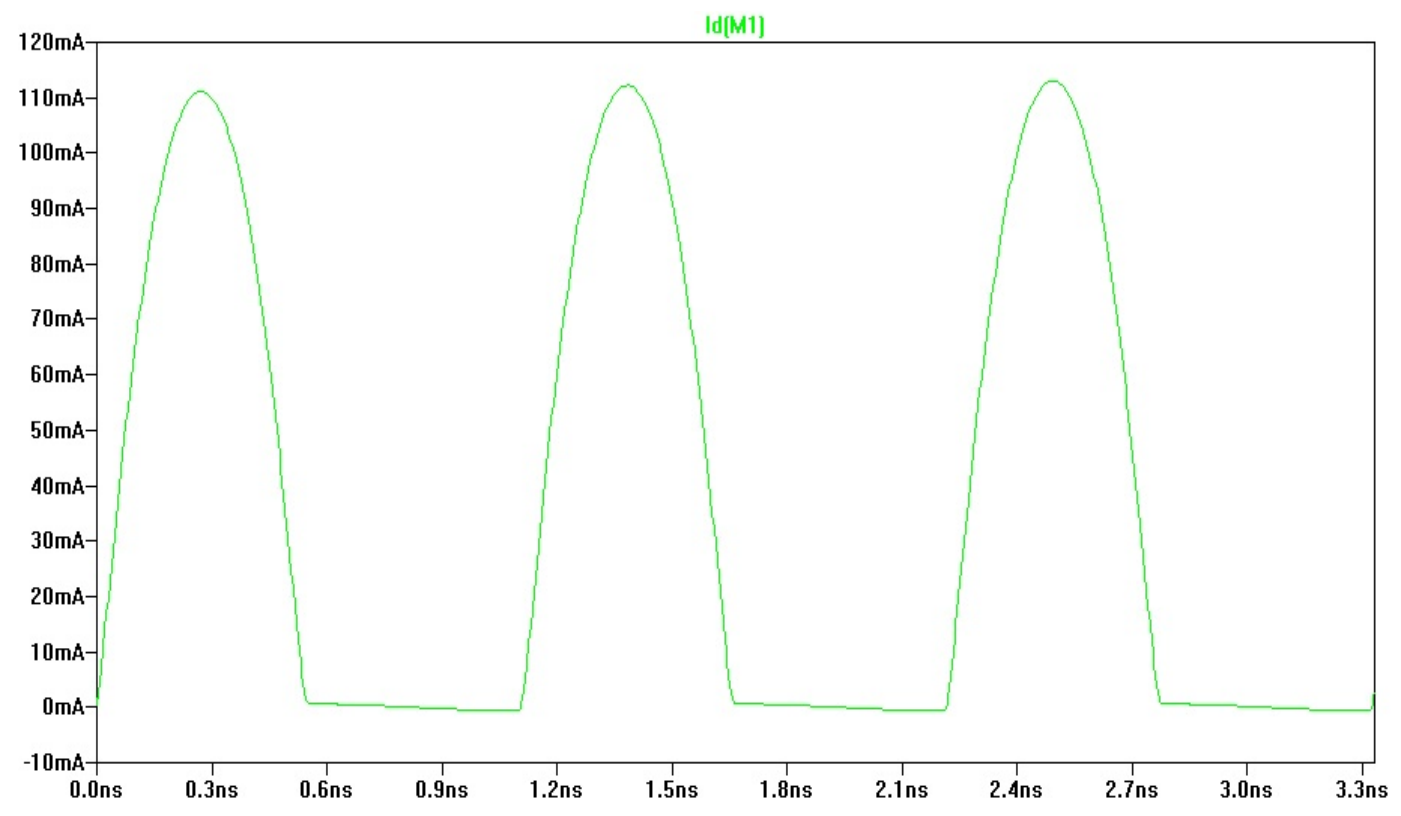

Figure 29: Drain current for $W=90 \mu m ~ L=180 \mathrm{~nm}$ 
The input power was measured by averaging $V_{\text {in }} \cdot l_{\text {in }}$ over 10 periods.

It was found to be $892.2 \mu \mathrm{W}$. The peak current through the drain was 114.2 mA. Verifying relationship (13) is satisfied

$$
I_{\text {max }}=114.2 \mathrm{~mA} \approx \frac{4 G P_{i}}{3 V}=\frac{400 \cdot 892.2 \mu \mathrm{W}}{3 \mathrm{~V}}=118.9 \mathrm{~mA}
$$

The FFT also shows that this input power is concentrated in the fundamental frequency.

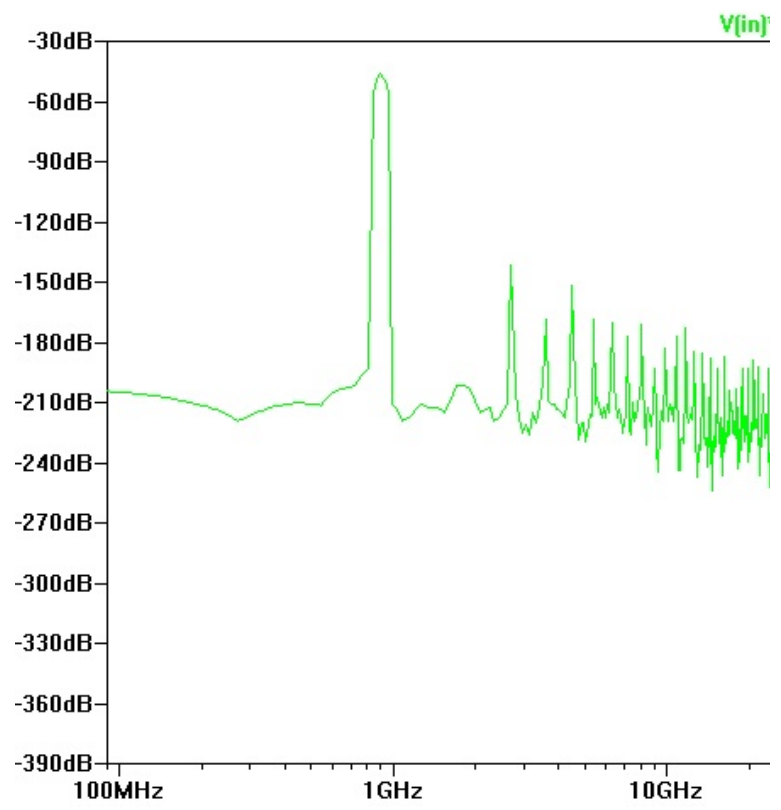

Figure 30: Input power FFT for $\mathrm{W}=90 \mu \mathrm{m} L=180 \mathrm{~nm}$ 
For the $5 / 1$ transistor the gain does not matter but the pole location and voltage divider ratio should be the same.

$$
\begin{gathered}
\left|\frac{V_{g}}{V_{\text {in }}}\right|=\frac{\frac{Q}{n}-\omega C_{\text {in }} R_{\text {bias }}}{\sqrt{1+\left(\frac{Q}{n}\right)^{2}}}=\frac{18-\frac{R_{\text {bias }}}{125 K \Omega}}{\sqrt{1+(18)^{2}}}=.78 \\
R_{\text {bias }}=492 \mathrm{~K} \Omega \approx 500 \mathrm{~K} \Omega \\
\frac{1}{R_{\text {bias }}\left(C_{\text {bypass }}+C_{\text {in }}\right)}=\frac{n \omega}{Q} \\
C_{\text {bypass }}=5.06 \mathrm{fF}
\end{gathered}
$$

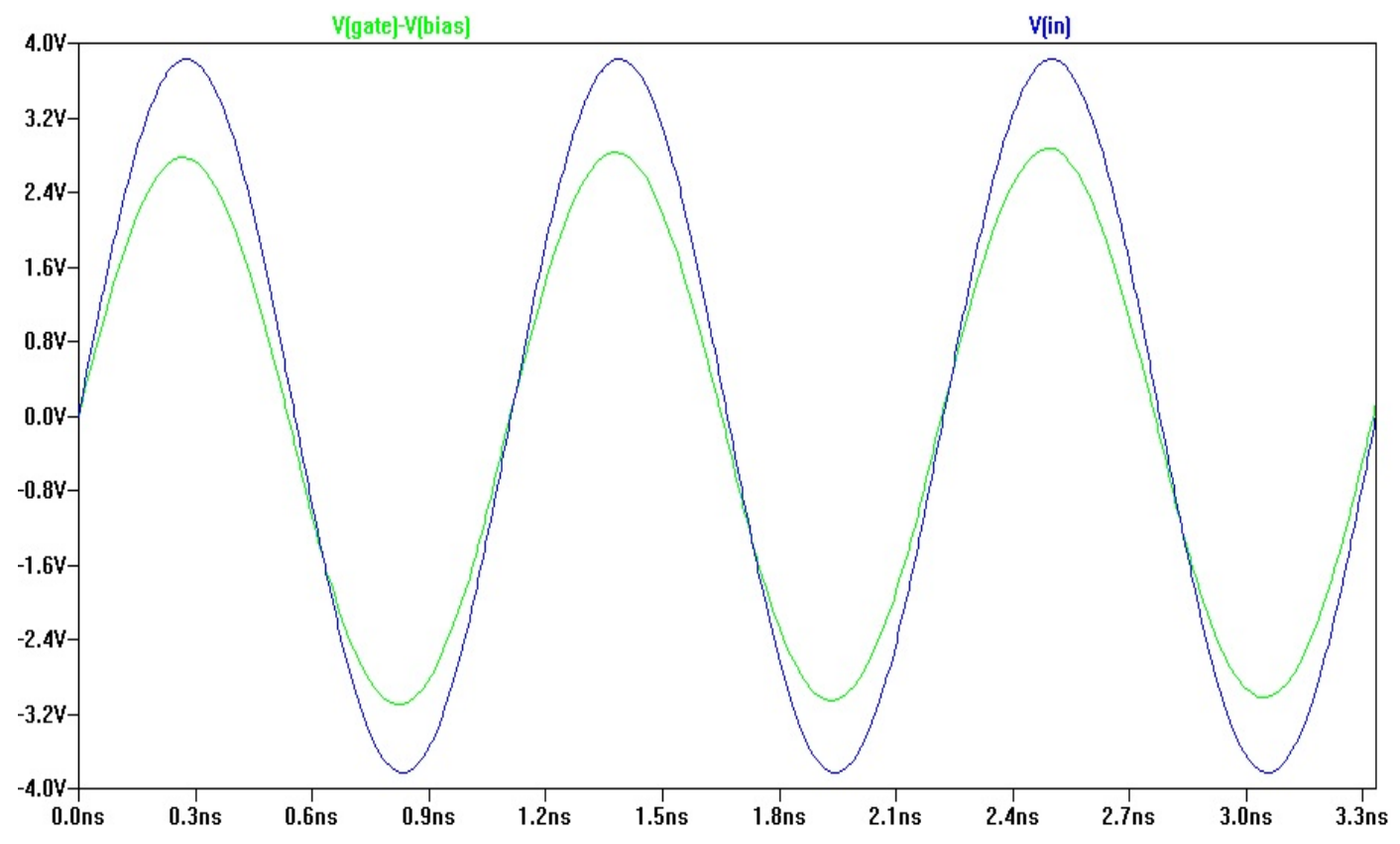

Figure 31: Input voltage and AC gate voltage for $W=900 \mathrm{~nm} L=180 \mathrm{~nm}$ 


\section{Input Network}

The input impedance to the entire system was measured and found to be 194 - j1100 $\Omega$. From this the values for the matching network components were calculated.

$$
\begin{gathered}
R_{s r c}=\left(R_{i}+j X_{i} \| j X_{L}\right)+j X_{c}=\frac{j R_{i} X_{L}-X_{L} X_{i}}{R_{i}+j X_{L}+j X_{i}}+j X_{C} \\
R_{s r c}-j X_{C}=\frac{j R_{i} X_{L}-X_{L} X_{i}}{R_{i}+j X_{L}+j X_{i}} \frac{R_{i}-j\left(X_{i}+X_{L}\right)}{R_{i}-j\left(X_{i}+X_{L}\right)} \\
\frac{R_{i} X_{L}^{2}+j\left(R^{2} X_{L}+X_{L} X_{i}^{2}+X_{L}^{2} X_{i}\right)}{R_{i}^{2}+\left(X_{L}+X_{i}\right)^{2}} \\
R_{s r c}=\frac{R_{i} X_{L}^{2}}{R_{i}^{2}+\left(X_{L}+X_{i}\right)^{2}} \\
-X_{C}=\frac{\left(R^{2} X_{L}+X_{L} X_{i}^{2}+X_{L}^{2} X_{i}\right)}{R_{i}^{2}+\left(X_{L}+j X_{i}\right)^{2}} \\
X_{L}=454=379 \\
X_{C}=-624=-564 \\
C=283 \mathrm{fF}=313 \mathrm{f} \\
L=80.3 \mathrm{nH}=67 \mathrm{nH} \\
C=6
\end{gathered}
$$

Since the input impedance to the $5 / 1$ transistors is so large the same input matching network will be used for the CCAB amplifiers and the NB amplifiers. 


\section{Output Matching Network}

Figure 32 shows the schematic for the output network of the 500/1 transistor.

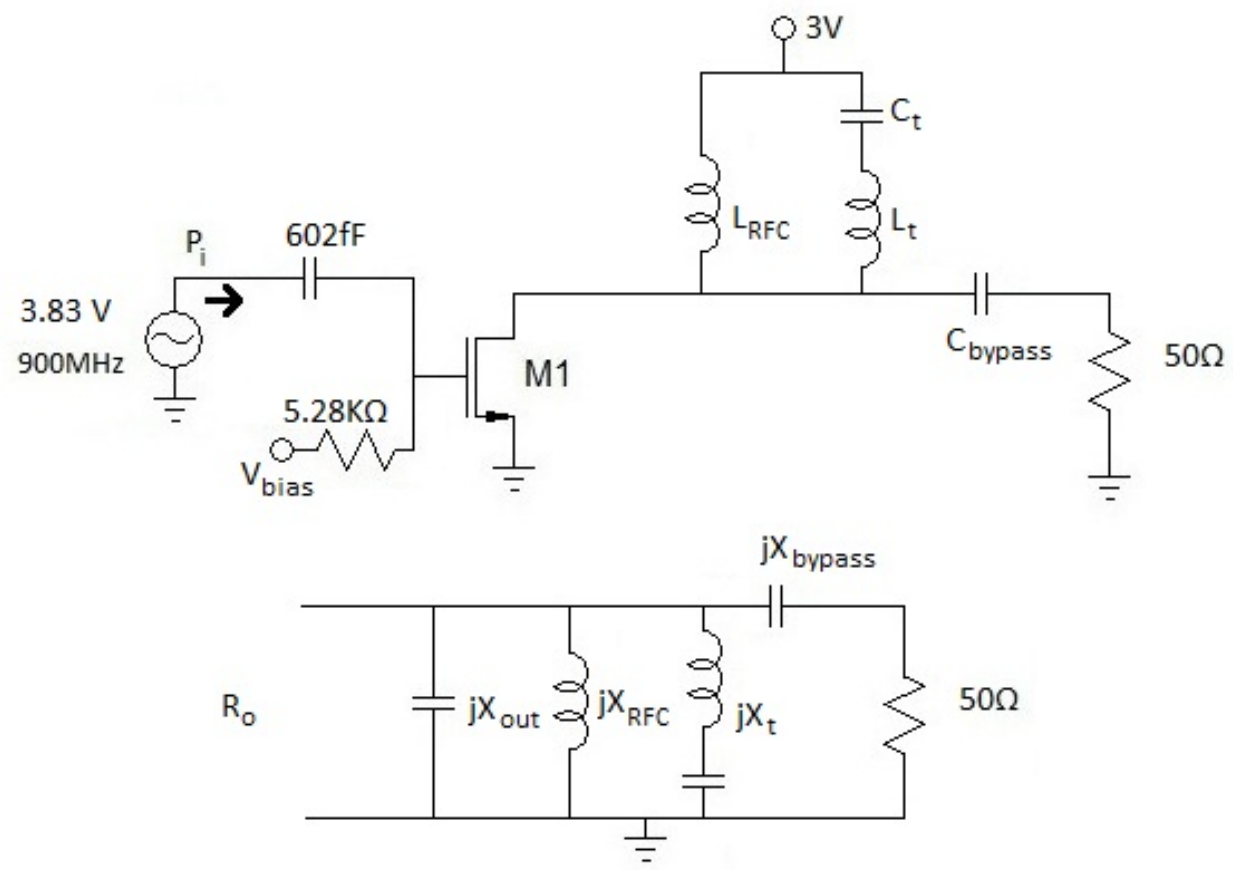

Figure 32: Output matching network schematic

For each conduction angle tested, a specific matching network will be used to obtain a full voltage swing at the saturation point.

$$
\begin{gathered}
V_{O N}=V_{A C} \cos \frac{\theta}{2}=V_{t}-V_{\text {bias }} \\
V_{\text {bias }}=0.5 V-3 V \cos \frac{\theta}{2} \\
V_{g \text { max }}=3 V+V_{\text {bias }} \\
I_{d \text { max }}=\left.I_{d}\right|_{V_{\text {max }}}
\end{gathered}
$$


Evaluating (2) at the peak

$$
\begin{gathered}
i_{d}=g_{m}\left(V_{A C} \cos \omega t-V_{A C} \cos \frac{\theta}{2}\right)=g_{m}\left(V_{A C}-V_{o n}\right)=I_{d \max } \\
g_{m} V_{A C}=I_{d \text { max }}+g_{m} V_{o n}
\end{gathered}
$$

From (5)

$$
\begin{gathered}
I_{\omega}=\frac{I_{d \max }+g_{m} V_{o n}}{\pi}\left(\frac{\theta}{2}+\frac{1}{2} \sin \theta-2 \cos \frac{\theta}{2} \sin \frac{\theta}{2}\right) \\
R_{o}=\frac{3 V}{I_{\omega}}
\end{gathered}
$$

We will test conduction angles of $\pi, 0.9 \pi, 0.7 \pi, 0.5 \pi$, and $0.3 \pi$

\begin{tabular}{|c|c|c|c|c|c|}
\hline$\theta$ & $\mathrm{V}_{\text {bias }}$ & $\mathrm{V}_{\mathrm{g} \max }$ & $\mathrm{I}_{\mathrm{d} \max }$ & $\mathrm{I}_{\omega}$ & $\mathrm{R}_{\circ}$ \\
\hline $180^{\circ}$ & 0.50 & 3.50 & 113.6 & 56.8 & 52.8 \\
\hline $162^{\circ}$ & 0.03 & 3.03 & 98.9 & 47.5 & 63.2 \\
\hline $126^{\circ}$ & -0.86 & 2.14 & 67.1 & 27.4 & 109.6 \\
\hline $90^{\circ}$ & -1.62 & 1.38 & 36.5 & 11.3 & 264.7 \\
\hline $54^{\circ}$ & -2.17 & 0.83 & 13.6 & 2.7 & 1131.7 \\
\hline
\end{tabular}

Table 4: Desired Output resistance for multiple conduction angles 
Calculating the required inductances and capacitances for the output network

$$
\begin{gathered}
X_{p}=X_{R F C}\left\|X_{t}\right\| X_{\text {out }} \\
R_{L}=\left(R_{o} \| j X_{p}\right)+j X_{\text {bypass }}=\frac{j R_{o} X_{p}}{R_{o}+j X_{p}}+j X_{\text {bypass }} \\
R_{L}-j X_{\text {bypass }}=\frac{j R_{o} X_{p}}{R_{o}+j X_{p}} \frac{R_{o}-j X_{p}}{R_{o}-j X_{p}}=\frac{j R_{o}^{2} X_{p}+R_{o} X_{p}^{2}}{R_{o}^{2}+X_{p}^{2}} \\
R_{L}=\frac{R_{o} X_{p}^{2}}{R_{o}^{2}+X_{p}^{2}} \\
X_{p}=\frac{\sqrt{R_{L}} R_{o}}{\sqrt{R_{o}-R_{L}}} \\
X_{\text {bypass }}=-\frac{R_{o}^{2} X_{p}}{R_{o}^{2}+X_{p}^{2}} \\
C_{\text {bypass }}=\frac{-1}{\omega X_{\text {bypass }}}
\end{gathered}
$$

To limit upper harmonics a series LC circuit resonant at $2 \omega$ is added in parallel

$$
\begin{aligned}
& L_{t}=\frac{20 R_{\text {out }}}{2 \omega} \\
& 2 \omega=\frac{1}{\sqrt{L_{t} C_{t}}} \\
& C_{t}=\frac{1}{L 4 \omega^{2}}
\end{aligned}
$$


At $\omega$ the reactance is

$$
\begin{gathered}
X_{t}=\omega L_{t}-\frac{1}{\omega C_{t}} \\
X_{p}=X_{R F C}\left\|X_{t}\right\| X_{\text {out }} \\
\frac{1}{X_{p}}=\frac{1}{X_{R F C}}+\frac{1}{X_{t} \| X_{\text {out }}} \\
X_{R F C}=\frac{\left(X_{t} \| X_{\text {out }}\right) X_{p}}{\left(X_{t} \| X_{\text {out }}\right)-X_{p}} \\
L_{R F C}=\omega X_{R F C}
\end{gathered}
$$

\begin{tabular}{|c|c|c|c|c|c|}
\hline$\theta$ & $\mathrm{R}_{\mathrm{o}}(\Omega)$ & $\mathrm{X}_{\mathrm{p}}(\Omega)$ & $\mathrm{X}_{\text {bypass }}(\Omega)$ & $\mathrm{X}_{\mathrm{t}}(\Omega)$ & $\mathrm{X}_{\mathrm{RFC}}(\Omega)$ \\
\hline $180^{\circ}$ & 52.8 & 222.5 & 11.9 & -79.2 & 57.7 \\
\hline $162^{\circ}$ & 63.2 & 123.0 & 25.7 & -94.8 & 53.0 \\
\hline $126^{\circ}$ & 109.6 & 100.4 & 54.6 & -164.4 & 61.5 \\
\hline $90^{\circ}$ & 264.7 & 127.7 & 103.6 & -397.1 & 94.8 \\
\hline $54^{\circ}$ & 1131.7 & 243.3 & 232.6 & -1697 & 204.0 \\
\hline
\end{tabular}

Table 5: Required output impedance values

\begin{tabular}{|c|c|c|c|c|}
\hline$\theta$ & $\mathrm{L}_{\mathrm{t}}(\mathrm{nH})$ & $\mathrm{C}_{\mathrm{t}}(\mathrm{fF})$ & $\mathrm{L}_{\mathrm{RFC}}(\mathrm{nH})$ & $\mathrm{C}_{\text {bypass }}(\mathrm{pF})$ \\
\hline $180^{\circ}$ & 4.67 & 1674 & 10.2 & 14.90 \\
\hline $162^{\circ}$ & 5.59 & 1399 & 9.4 & 6.88 \\
\hline $126^{\circ}$ & 9.69 & 807 & 10.9 & 3.24 \\
\hline $90^{\circ}$ & 23.4 & 334 & 16.8 & 1.71 \\
\hline $54^{\circ}$ & 100 & 78.1 & 36.1 & 0.76 \\
\hline
\end{tabular}

Table 6: Required output component values 


\section{Biasing Circuitry}

When the conduction angle of the class B amplifier was analyzed it was found that the gain remains constant even when the conduction angle begins to exceed $180^{\circ}$. This is because the transfer char acteristic does not truly follow the sudden turn on approximation. At the bias point it is actually on the whole time for small voltage swings, but the $g_{m}$ for the device at this point is only half of the $g_{m}$ in the linear range. This decrease in $g_{m}$ opposes the increase in conduction angle and keeps the gain constant.

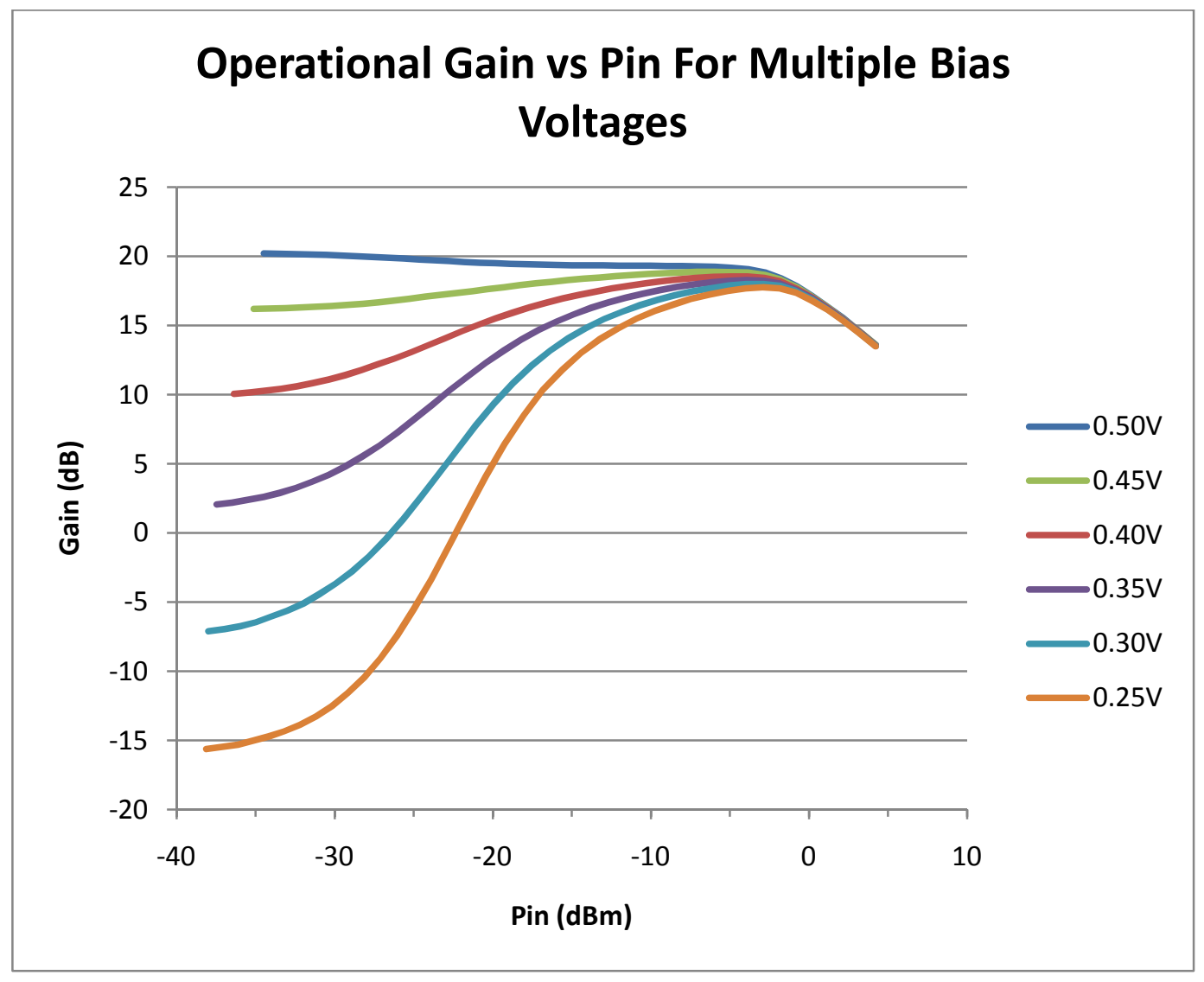

Figure 33: Operational gain of class B amplifier for different bias voltages 


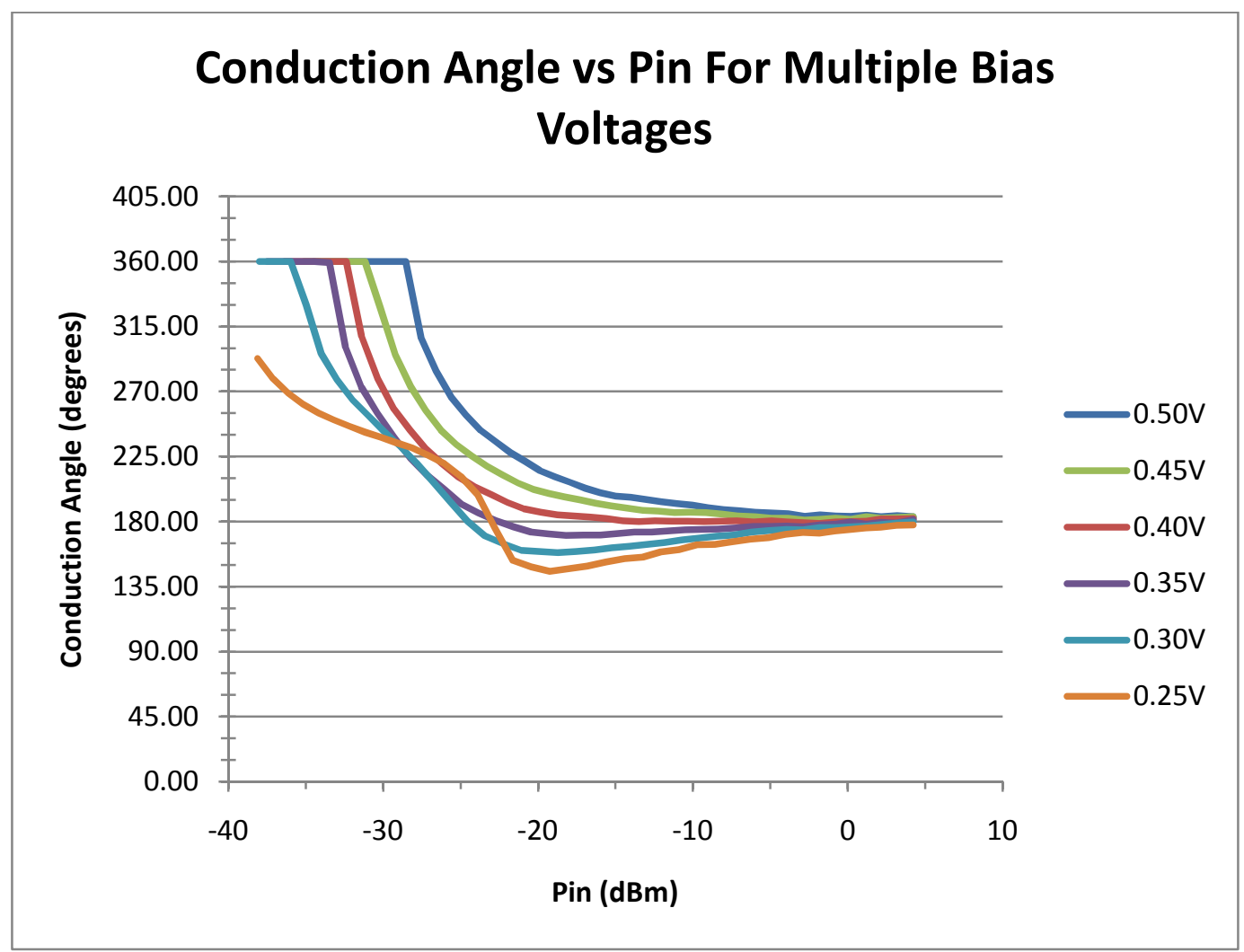

Figure 34: Conduction angle of class B amplifier for different bias voltages

Since both the gain and the conduction angle of the class B amp need to remain as constant as possible for the feedback system to be effective it was decided to bias the class B amplifier at $0.4 \mathrm{~V}$ instead of $0.5 \mathrm{~V}$. For $0.4 \mathrm{~V}$ bias the amplifier fluctuates by $3 \mathrm{~dB}$ and $6.5^{\circ}$ over the range of -20 to $0 \mathrm{dBm}$. 
From (6) the required ratio for the resistors is

$$
\begin{gathered}
\frac{I_{C C}}{I_{C B}}=\frac{V_{C C} / R_{2}}{V_{C B} / R_{1}}=\frac{R_{1}}{R_{2}}=\sin \frac{\theta}{2}-\frac{\theta}{2} \cos \frac{\theta}{2} \\
n=\frac{R_{2}}{R_{1}}=\frac{1}{\sin \frac{\theta}{2}-\frac{\theta}{2} \cos \frac{\theta}{2}} \\
R_{2}=n R_{1}
\end{gathered}
$$

To keep the poles in the same location

$$
\begin{gathered}
\omega=R_{1} C_{1}=R_{2} C_{2}=n R_{1} C_{2} \\
C_{2}=\frac{C_{1}}{n}
\end{gathered}
$$

The DC current for the class B transistor should peak at $0.56 \mathrm{~mA}$.

The voltage output from the trans-impedance amplifier should be less than $3 \mathrm{~V}$ so that an Op amp operating on $\pm 3 \mathrm{~V}$ rails can handle the biasing. With $\mathrm{R}_{1}=4 \mathrm{~K} \Omega, \mathrm{V}=0.56 \mathrm{~mA} \cdot 4 \mathrm{~K} \Omega=2.24 \mathrm{~V}$, which should be acceptable.

$$
C_{1}=\frac{1}{R_{1} \omega_{1}}=2 p F
$$

\begin{tabular}{|c|c|c|}
\hline$\theta$ & $\mathrm{R} 2$ & $\mathrm{C} 2$ \\
\hline $180^{\circ}$ & $4.00 \mathrm{~K} \Omega$ & $2.00 \mathrm{pF}$ \\
\hline $162^{\circ}$ & $5.22 \mathrm{~K} \Omega$ & $1.53 \mathrm{pF}$ \\
\hline $126^{\circ}$ & $10.2 \mathrm{~K} \Omega$ & $784 \mathrm{fF}$ \\
\hline $90^{\circ}$ & $26.3 \mathrm{~K} \Omega$ & $303 \mathrm{fF}$ \\
\hline $54^{\circ}$ & $117 \mathrm{~K} \Omega$ & $68 \mathrm{fF}$ \\
\hline
\end{tabular}

Table 7: Component values for lowpass filter 
The gain in any feedback system affects the poles of the closed loop response. The entire amplifier was simulated for each conduction angle setting and the biasing voltage was examined for a $0.7 V_{p}$ input, which corresponds to approximately $3 V_{p}$ at the gate of the transistors. The gain of the difference amplifier was tweaked for each setting to achieve a response with approximately the same damping factor. The voltage of the class $\mathrm{C}$ bias is shown in Figure 35 .

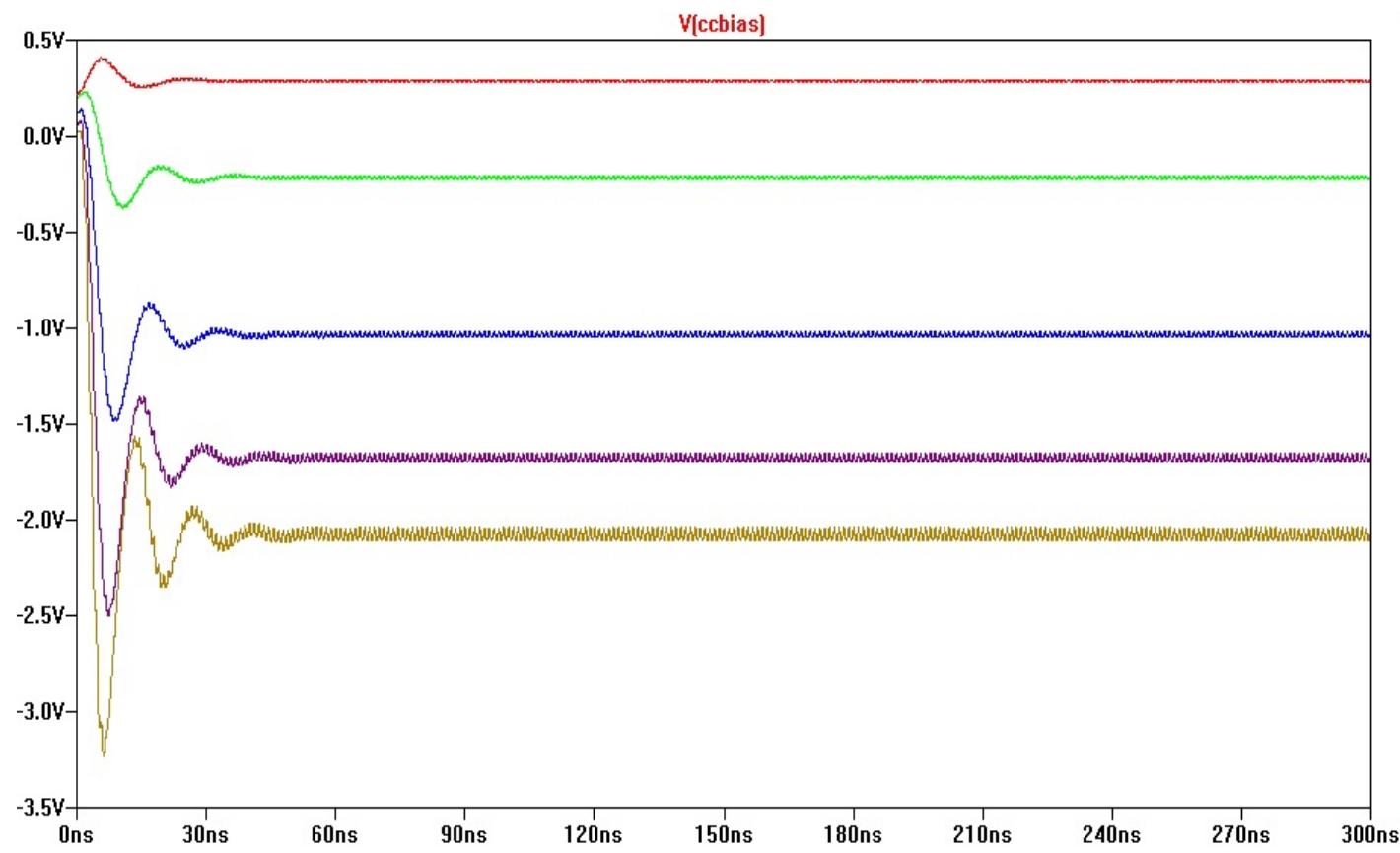

Figure 35: Class $\mathrm{C}$ bias voltage for different angle settings 


\begin{tabular}{|c|c|}
\hline$\theta$ & $\mathrm{K}$ \\
\hline $180^{\circ}$ & 6 \\
\hline $162^{\circ}$ & 5.5 \\
\hline $126^{\circ}$ & 3.2 \\
\hline $90^{\circ}$ & 1.75 \\
\hline $54^{\circ}$ & 0.6 \\
\hline
\end{tabular}

Table 8: DC gain for difference amplifier 


\section{Simulation Results}

\section{Measurement Procedure}

Figure 36 shows a standard 2 port network.

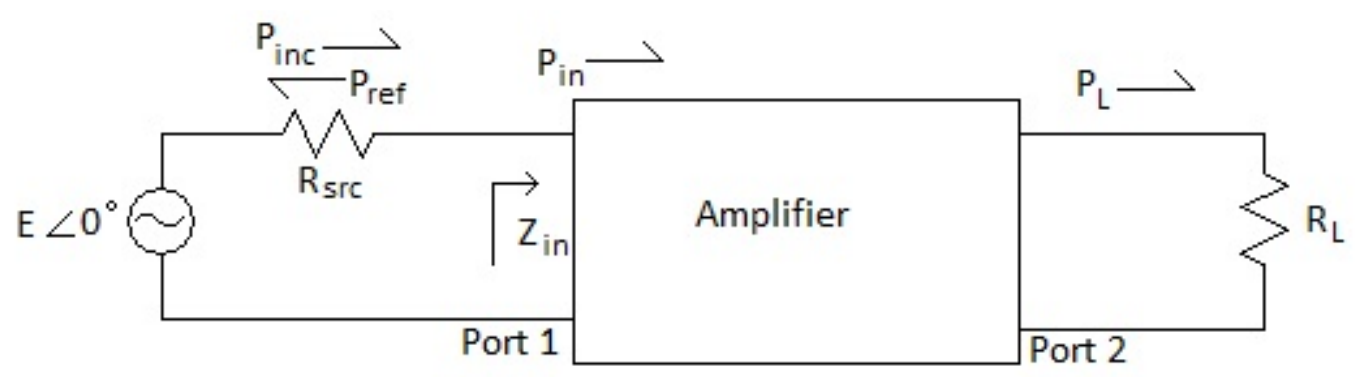

Figure 36: Two port network representation

While $l_{\text {in }}$ might have upper harmonics due to non linearity $V_{\text {in }}$ should only contain the fundamental harmonic. Assuming this is the case the power should only contain the fundamental harmonic as well.

$$
P_{i n}=\frac{1}{n T} \int_{t_{o}}^{t_{o}+n T} I_{1}(t) \cdot V_{1}(t) d t
$$

By averaging the product of the input current and input voltage for 10 periods the input power was obtained.

It will also be assumed that the power transmitted to the load will be dominated by the fundamental harmonic, since the matching network was designed to attenuate the second harmonic. The power transmitted to the load will be found by averaging the product of the current through the load and the voltage across the load over 10 periods. 
The DC power supplied to the drain will be found from averaging the product of the current from the supply for the drain and the 3V DC supply for the drain over 10 periods.

To find the incident power

$$
\begin{aligned}
& P_{\text {in }}=\frac{1}{2} \operatorname{Re}\left(V_{1} I_{1}^{*}\right)=\frac{1}{2} \operatorname{Re}\left(\frac{E Z_{\text {in }}}{\left(R_{\text {src }}+Z_{\text {in }}\right)} \frac{E^{*}}{\left(R_{\text {src }}+Z_{\text {in }}\right)^{*}}\right)=\frac{1}{2} \frac{|E|^{2}}{\left|R_{\text {src }}+Z_{\text {in }}\right|^{2}} \operatorname{Re}\left(Z_{\text {in }}\right) \\
& =\frac{|E|^{2} R_{\text {in }}}{2\left|R_{\text {src }}+Z_{\text {in }}\right|^{2}} \\
& V_{1}=V_{1}^{+}+V_{1}^{-} \\
& I_{1}=\frac{V_{1}^{+}}{Z_{o}}-\frac{V_{1}^{-}}{Z_{o}} \\
& Z_{o}=R_{s r c} \\
& P_{\text {in }}=\frac{1}{2} \operatorname{Re}\left(V_{1} I_{1}^{*}\right)=\frac{1}{2} \operatorname{Re}\left(\left(V_{1}^{+}+V_{1}^{-}\right)\left(\frac{V_{1}^{+}}{R_{s r c}}-\frac{V_{1}^{-}}{R_{s r c}}\right)^{*}\right) \\
& =\frac{1}{2 R_{\text {src }}}\left(V_{1}^{+2}-V_{1}^{-2}\right)=P_{\text {inc }}-P_{\text {ref }}=P_{i n c}-|\Gamma|^{2} P_{i n c} \\
& P_{\text {inc }}=\frac{P_{\text {in }}}{1-|\Gamma|^{2}} \\
& \Gamma=\frac{Z_{\text {in }}-R_{\text {src }}}{Z_{\text {in }}+R_{\text {src }}} \\
& 1-|\Gamma|^{2}=1-\frac{\left|Z_{\text {in }}-R_{s r c}\right|^{2}}{\left|Z_{\text {in }}+R_{s r c}\right|^{2}}=\frac{\left|Z_{\text {in }}+R_{s r c}\right|^{2}-\left|Z_{\text {in }}-R_{s r c}\right|^{2}}{\left|Z_{\text {in }}+R_{s r c}\right|^{2}} \\
& =\frac{\left(R_{s r c}+R_{i n}\right)^{2}+X_{L}^{2}-\left(R_{\text {in }}-R_{s r c}\right)^{2}-X_{L}^{2}}{\left|Z_{\text {in }}+R_{s r c}\right|^{2}}=\frac{4 R_{\text {in }} R_{s r c}}{\left|Z_{\text {in }}+R_{s r c}\right|^{2}}
\end{aligned}
$$




$$
\begin{gathered}
P_{i n c}=\frac{P_{i n}}{1-|\Gamma|^{2}}=\frac{\left(\frac{|E|^{2} R_{i n}}{2\left|R_{s r c}+Z_{i n}\right|^{2}}\right)}{\left(\frac{4 R_{i n} R_{s r c}}{\left|Z_{\text {in }}+R_{s r c}\right|^{2}}\right)} \\
P_{i n c}=\frac{|E|^{2}}{8 R_{s r c}}
\end{gathered}
$$

With the input voltage, input power, transmitted power and DC power measured from the simulation the transducer power gain, operational power gain, VSWR, and drain efficiency can all be calculated.

$$
\begin{gathered}
G_{T}=\frac{P_{L}}{P_{i n c}} \\
G_{p}=\frac{P_{L}}{P_{i n}} \\
|\Gamma|=\sqrt{1-\frac{P_{i n}}{P_{i n c}}} \\
V S W R=\frac{1+|\Gamma|}{1-|\Gamma|} \\
\eta=\frac{P_{L}}{P_{D C}}
\end{gathered}
$$


The conduction angle can also be directly measured from the simulation.

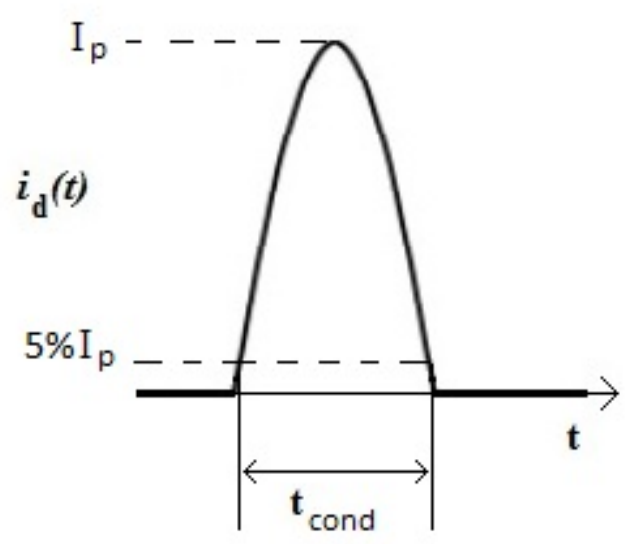

Figure 37: Definition for $t_{\text {cond }}$

$$
\theta_{\text {cond }}=360^{\circ} \cdot \frac{t_{\text {cond }}}{T}
$$

The magnitude of the input voltage was swept over $40 \mathrm{~dB}$, for all five specified conduction angle settings, to both the CCAB amplifiers and NB amplifiers. The desired measurements were taken starting at 200ns, when the amplifier had reached steady state.

Additional testing of the impact of the feedback gain, single tone frequency response, AM modulated input response, and FM modulated input response will be performed for the $126^{\circ}$ bias set ting which demonstrated a good trade-off between linearity and efficiency. 


\section{Data}

Figure 38 and Figure 39 show the conduction angle for the NB amplifiers.

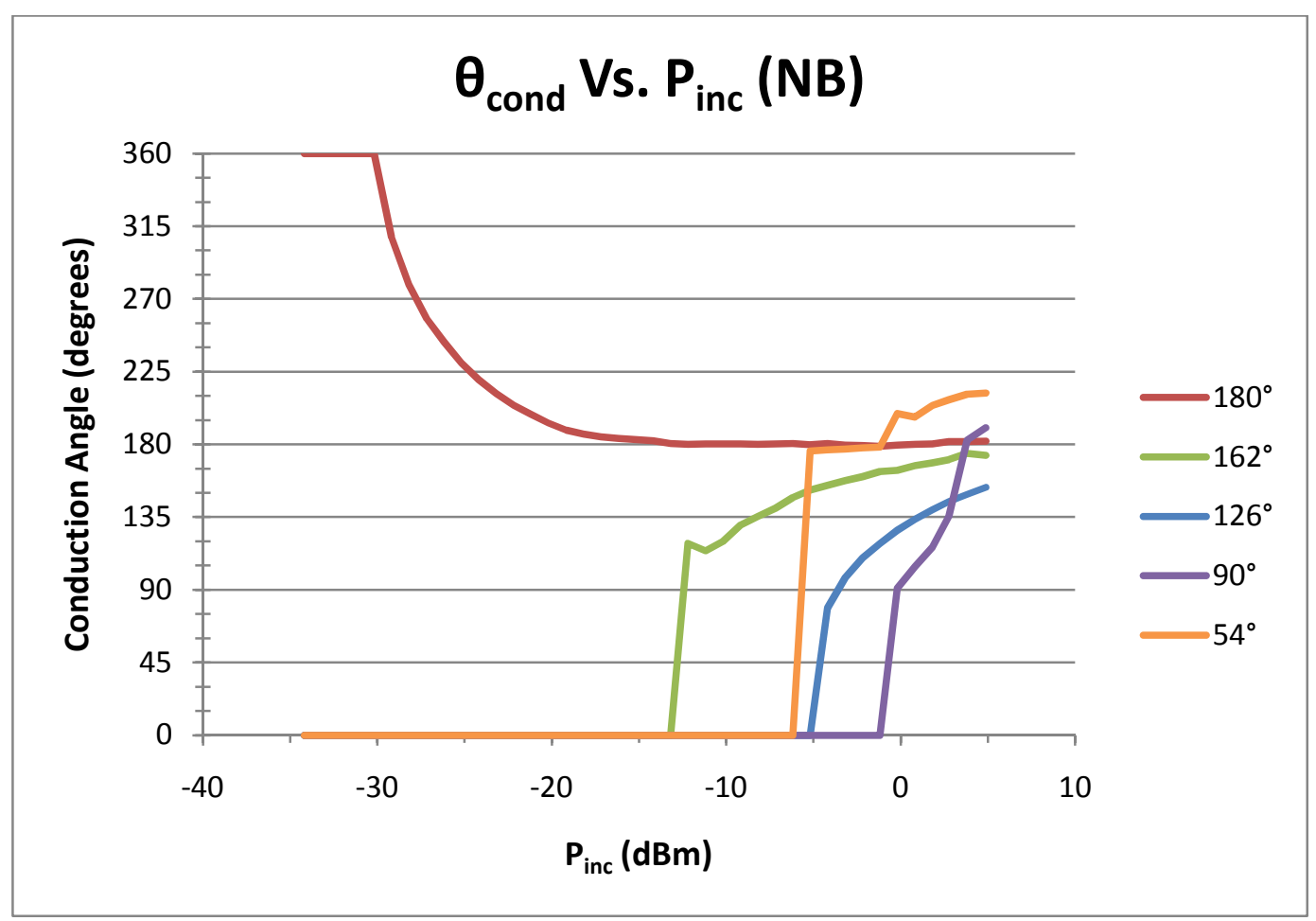

Figure 38: Conduction angle for NB amplifiers over $40 \mathrm{~dB}$ range 


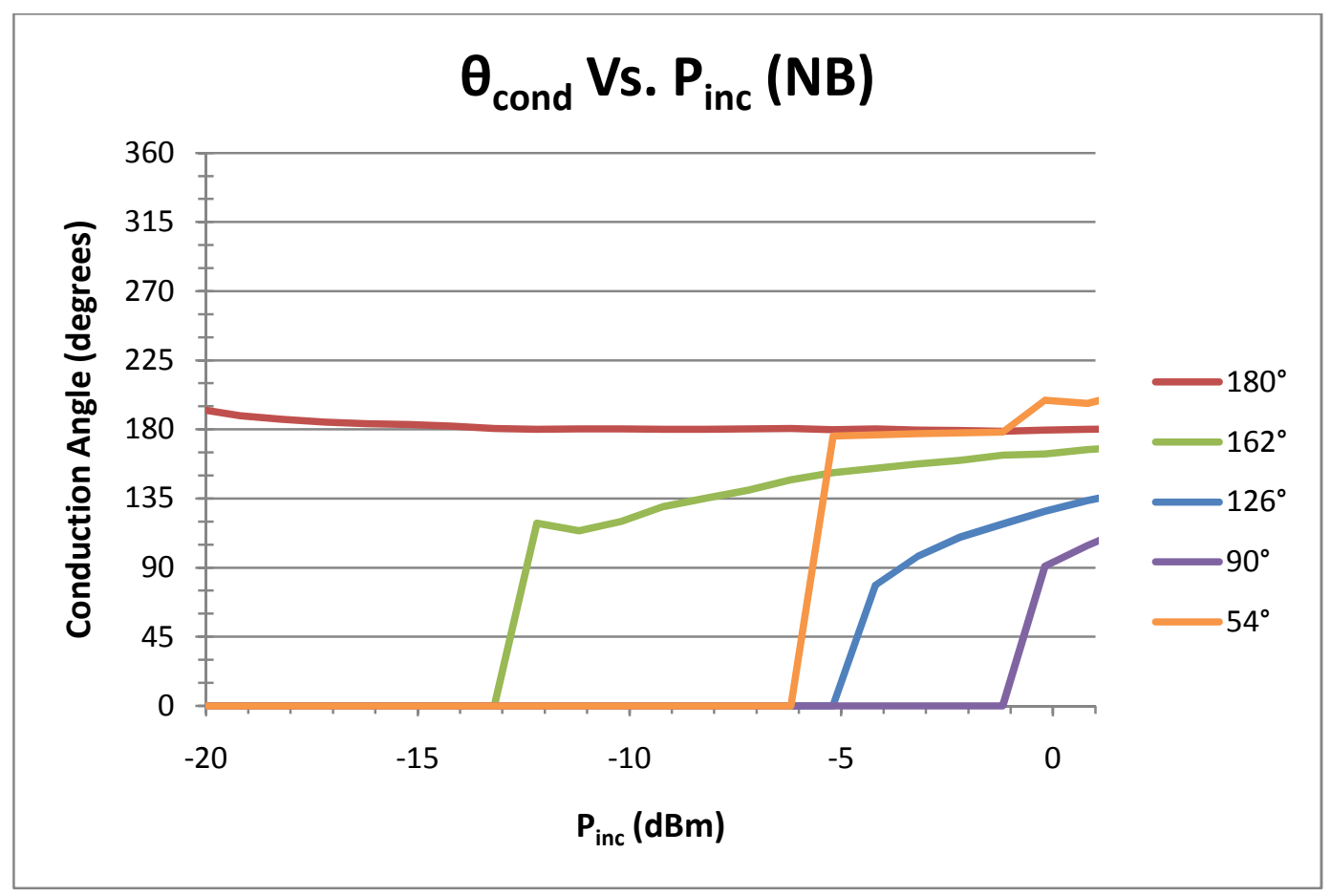

Figure 39: Conduction angle for NB amplifiers over $20 \mathrm{~dB}$ range

Figure 40 and Figure 41 show the conduction angle for the $C C A B$ amplifiers. 


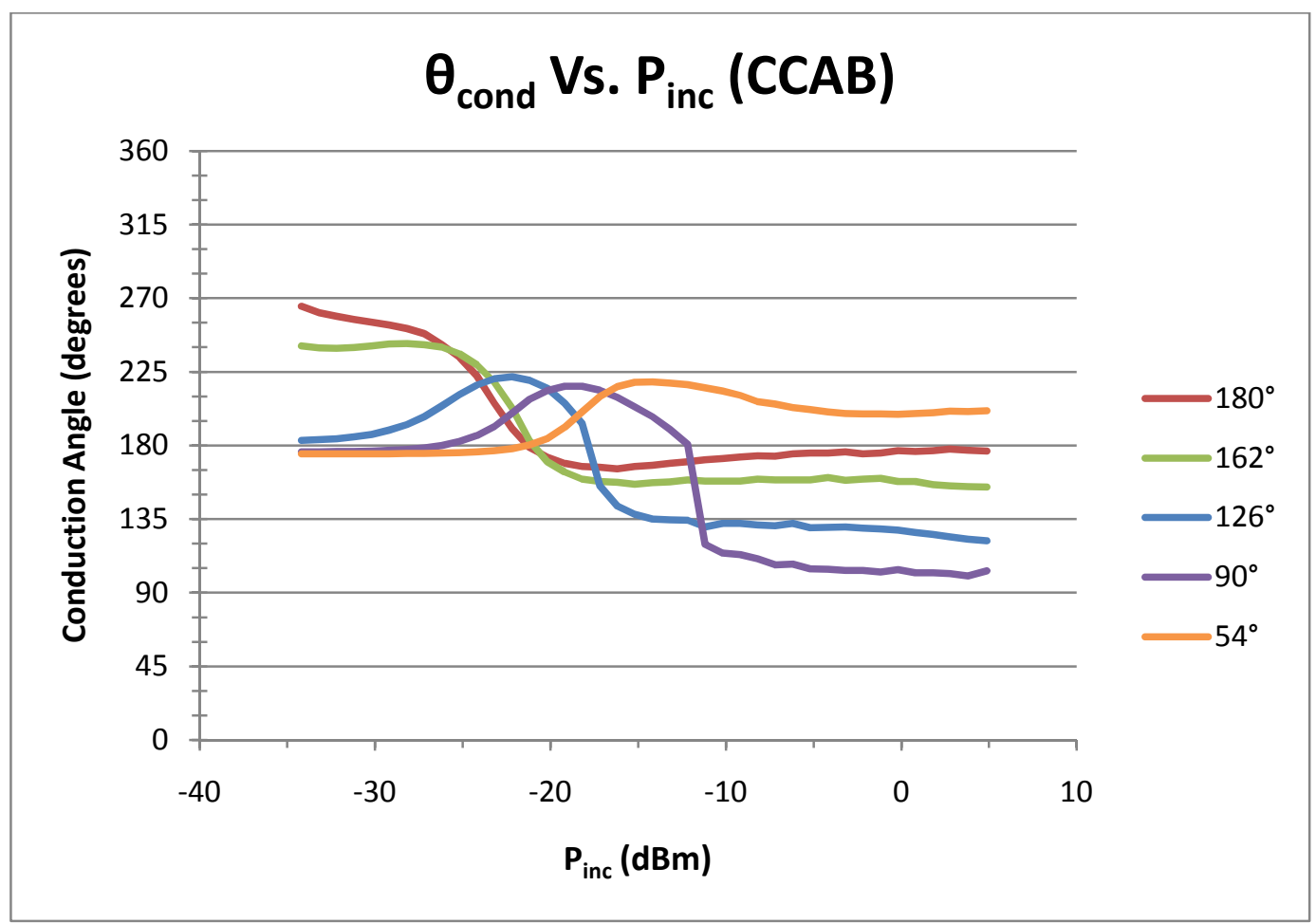

Figure 40: Conduction angle for CCAB amplifiers over $40 \mathrm{~dB}$ range

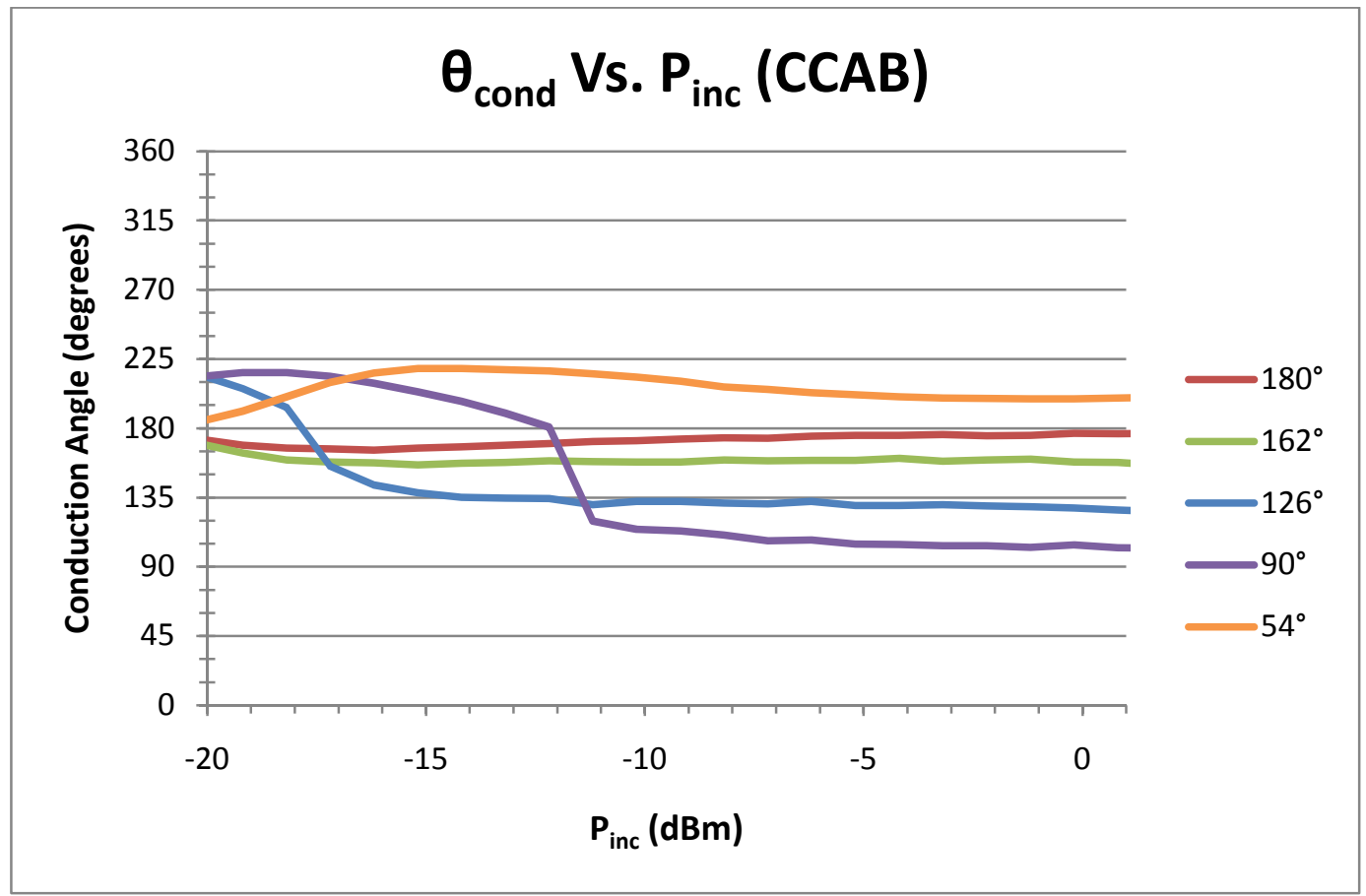

Figure 41: Conduction angle for $C C A B$ amplifiers over $20 \mathrm{~dB}$ range 
The conduction angle for the $180^{\circ}, 162^{\circ}$, and $126^{\circ} \mathrm{C}$ CAB amplifiers stay within $5 \%$ of the expected value for over $12 \mathrm{~dB}$. The $90^{\circ} \mathrm{CCAB}$ amplifier fluctuates by $20 \%$ in the same $12 \mathrm{~dB}$ range and is also offset from the expected $90^{\circ}$ value by over $10 \%$. The $54^{\circ} \mathrm{CCA} B$ amplifier operates with the peak of the input wave so close to the turn on voltage, that the sharp turn on approximation does not hold and the conduction angle is actually above $180^{\circ}$.

Figure 42 and Figure 43 show the transducer gain for the NB amplifiers.

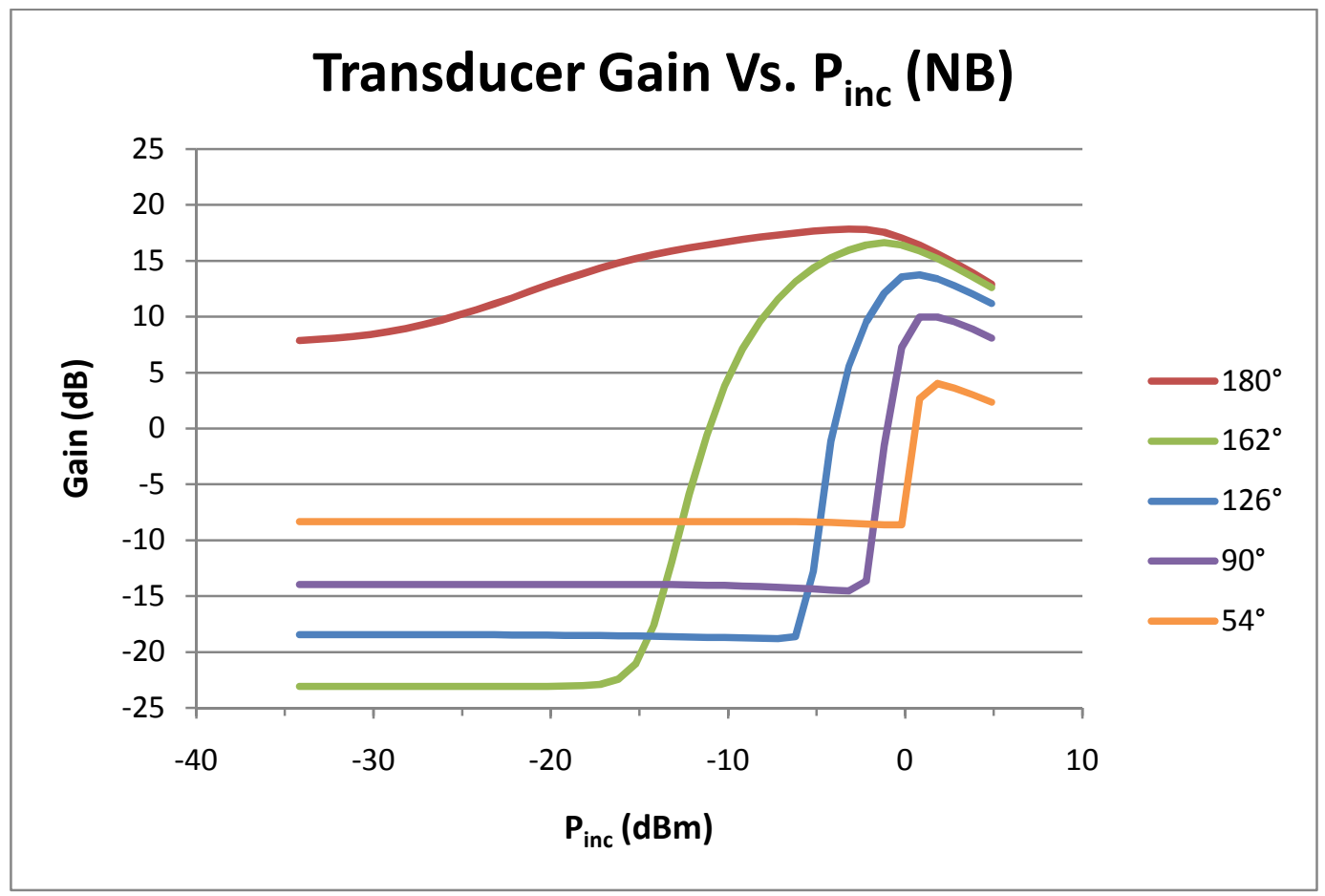

Figure 42: Transducer gain for NB amplifiers over $40 \mathrm{~dB}$ range 


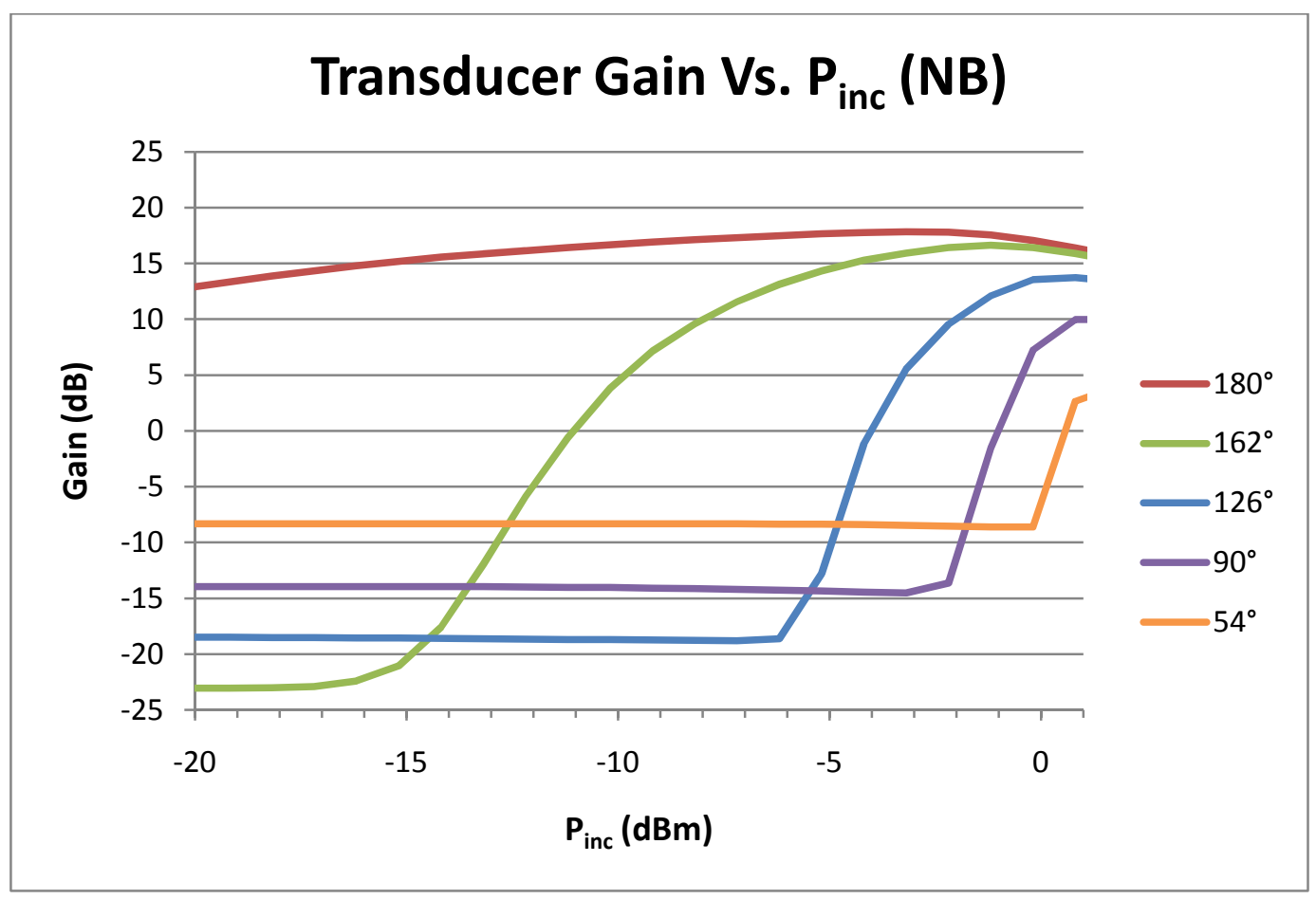

Figure 43: Transducer gain for NB amplifiers over $20 \mathrm{~dB}$ range

Figure 44 and Figure 45 show the transducer gain for the CCAB amplifiers. 


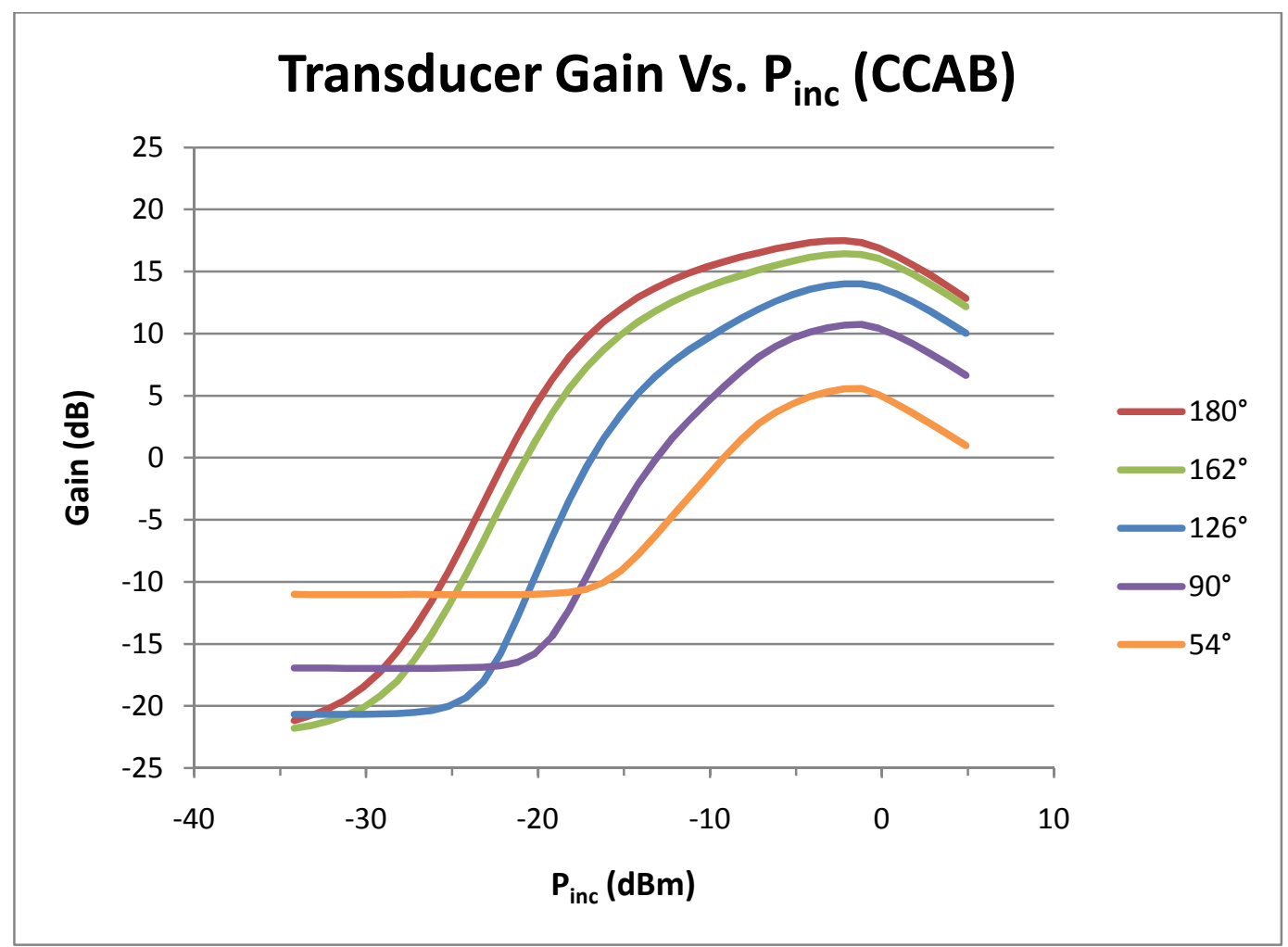

Figure 44: Transducer gain for $C$ CAB amplifiers over $40 \mathrm{~dB}$ range

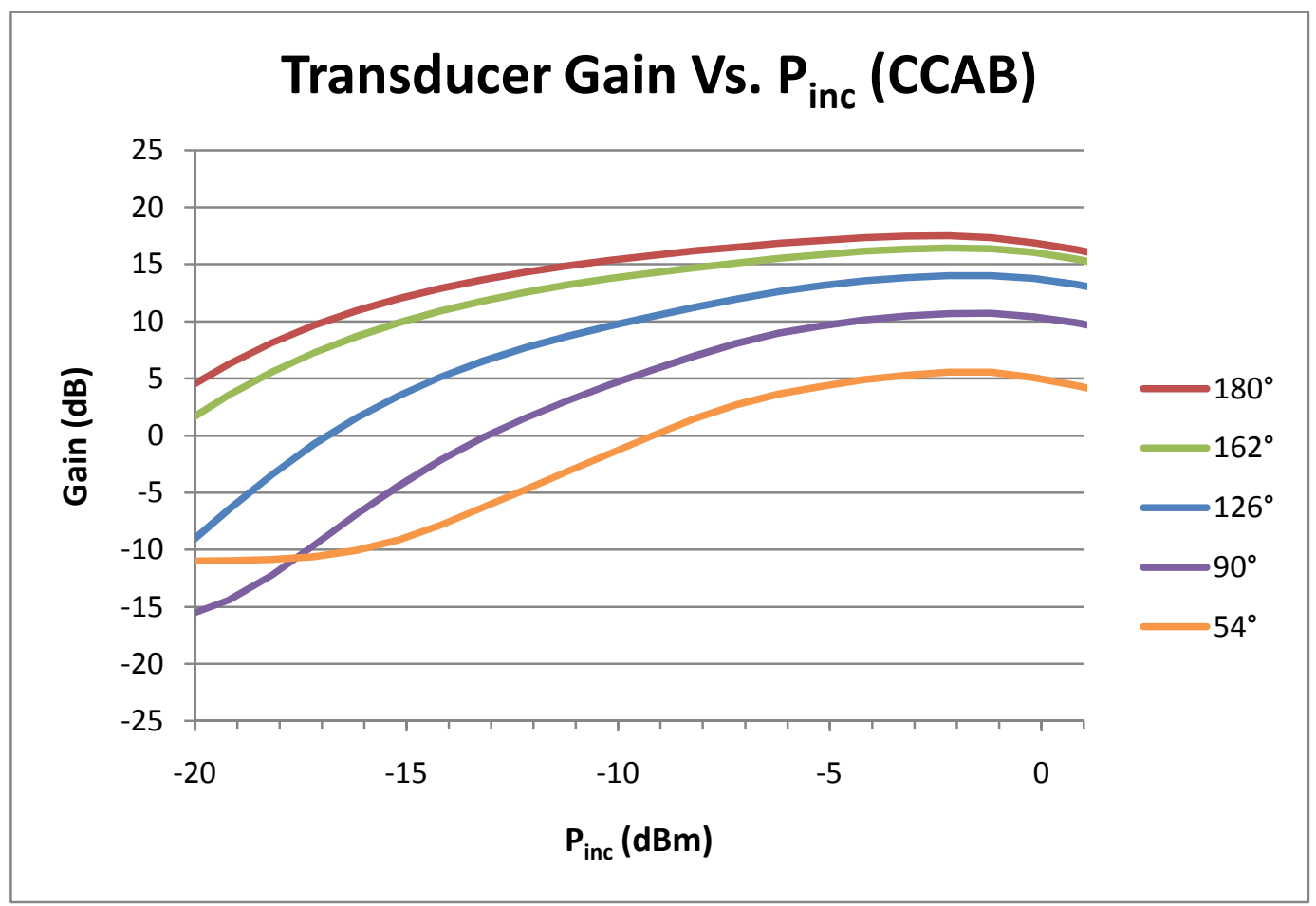

Figure 45: Transducer gain for CCAB amplifiers over $20 \mathrm{~dB}$ range 
The CCAB amplifiers keep the gain less than $3 \mathrm{~dB}$ below the peak gain for a range of over $8 \mathrm{~dB}$ below the $1 \mathrm{~dB}$ compression point for all five angle settings. In contrast the NB amplifiers have this range for less than 3 $\mathrm{dB}$ for the settings below $162^{\circ}$.

Figure 46 and Figure 47 show the VSWR for the NB amplifiers.

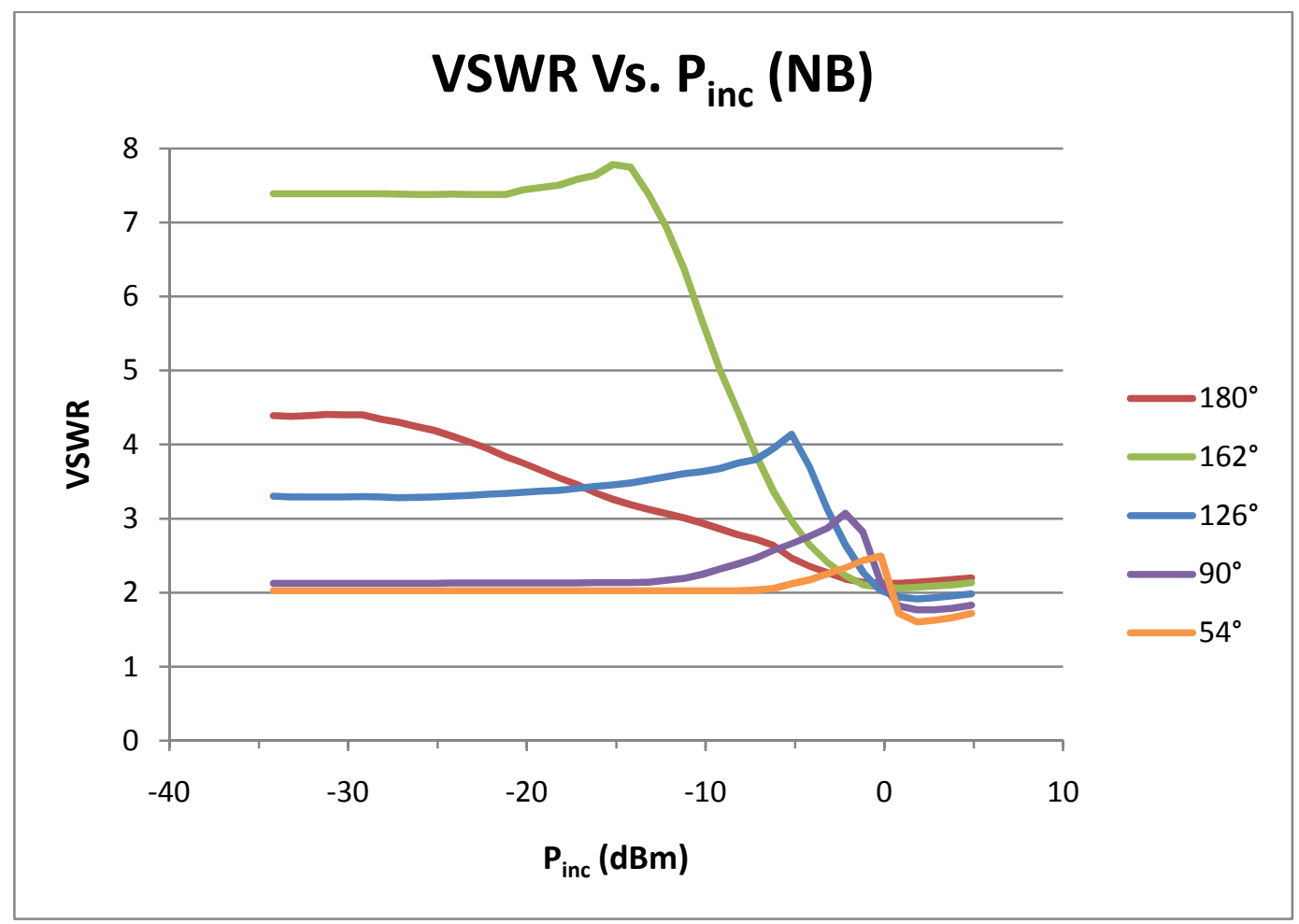

Figure 46: VSWR for NB amplifiers over $40 \mathrm{~dB}$ range 


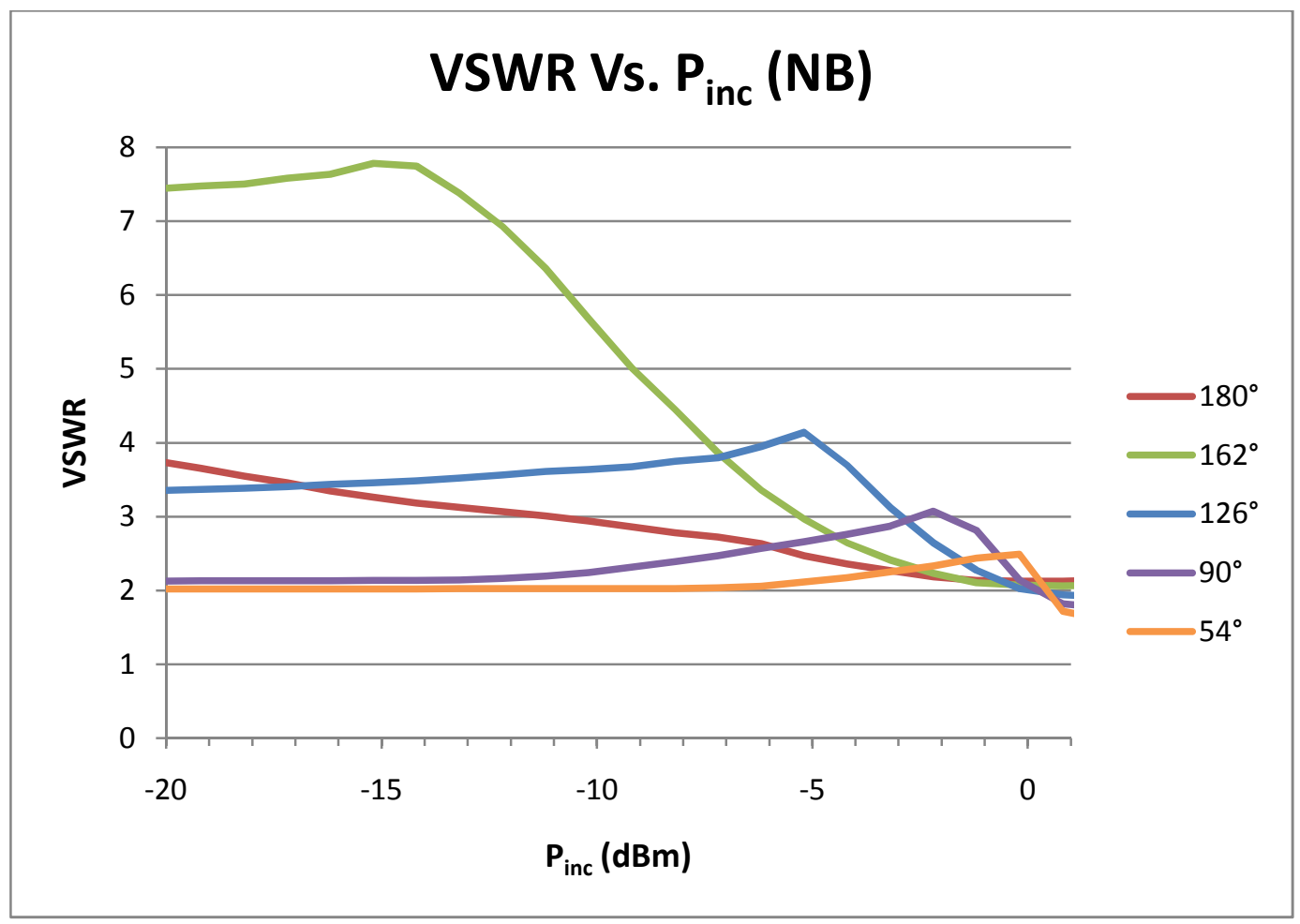

Figure 47: VSWR for NB amplifiers over $20 \mathrm{~dB}$ range

Figure 48 and Figure 49 show the VSWR for the CCAB amplifiers 


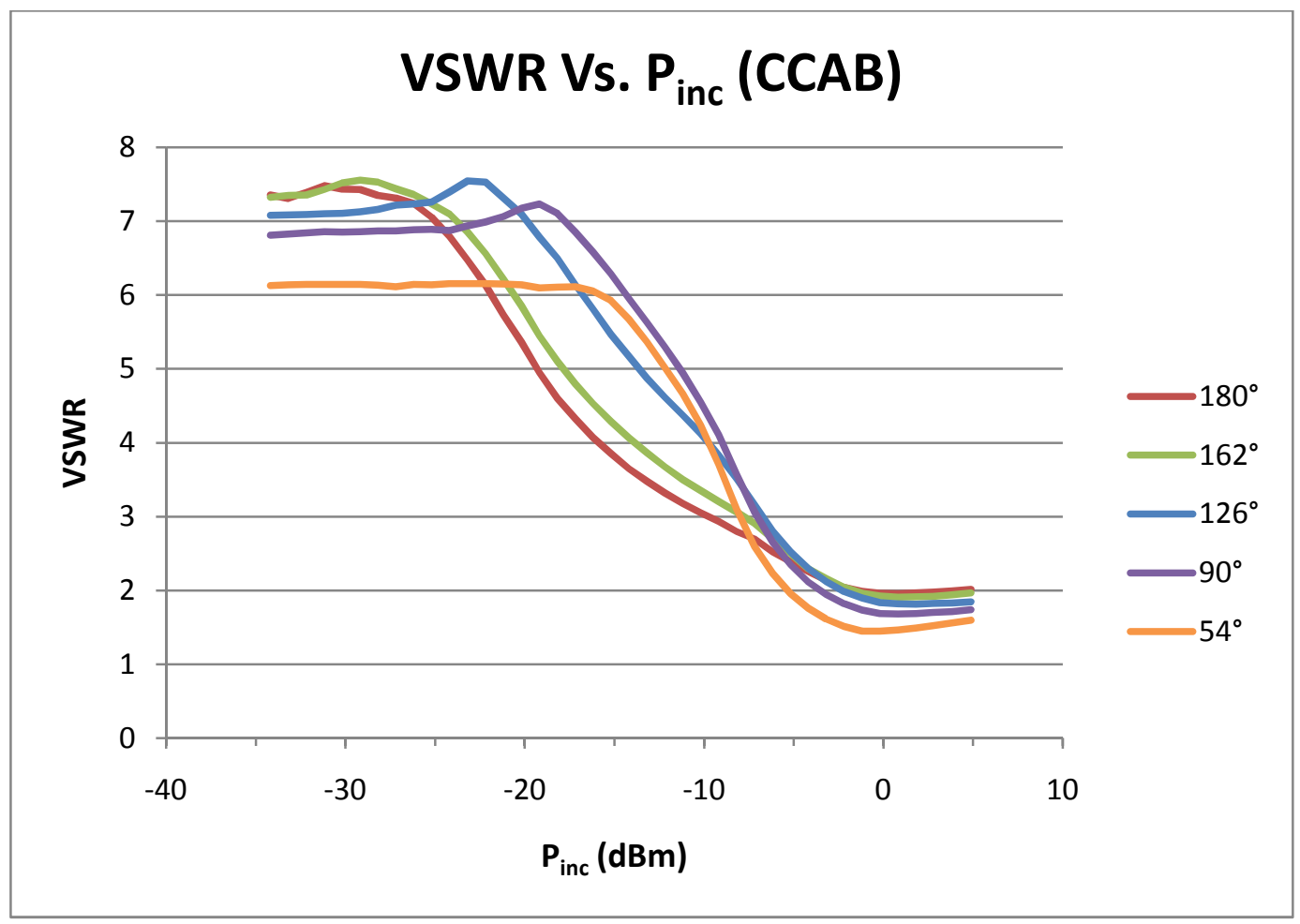

Figure 48: VSWR for CCAB amplifiers over $40 \mathrm{~dB}$ range

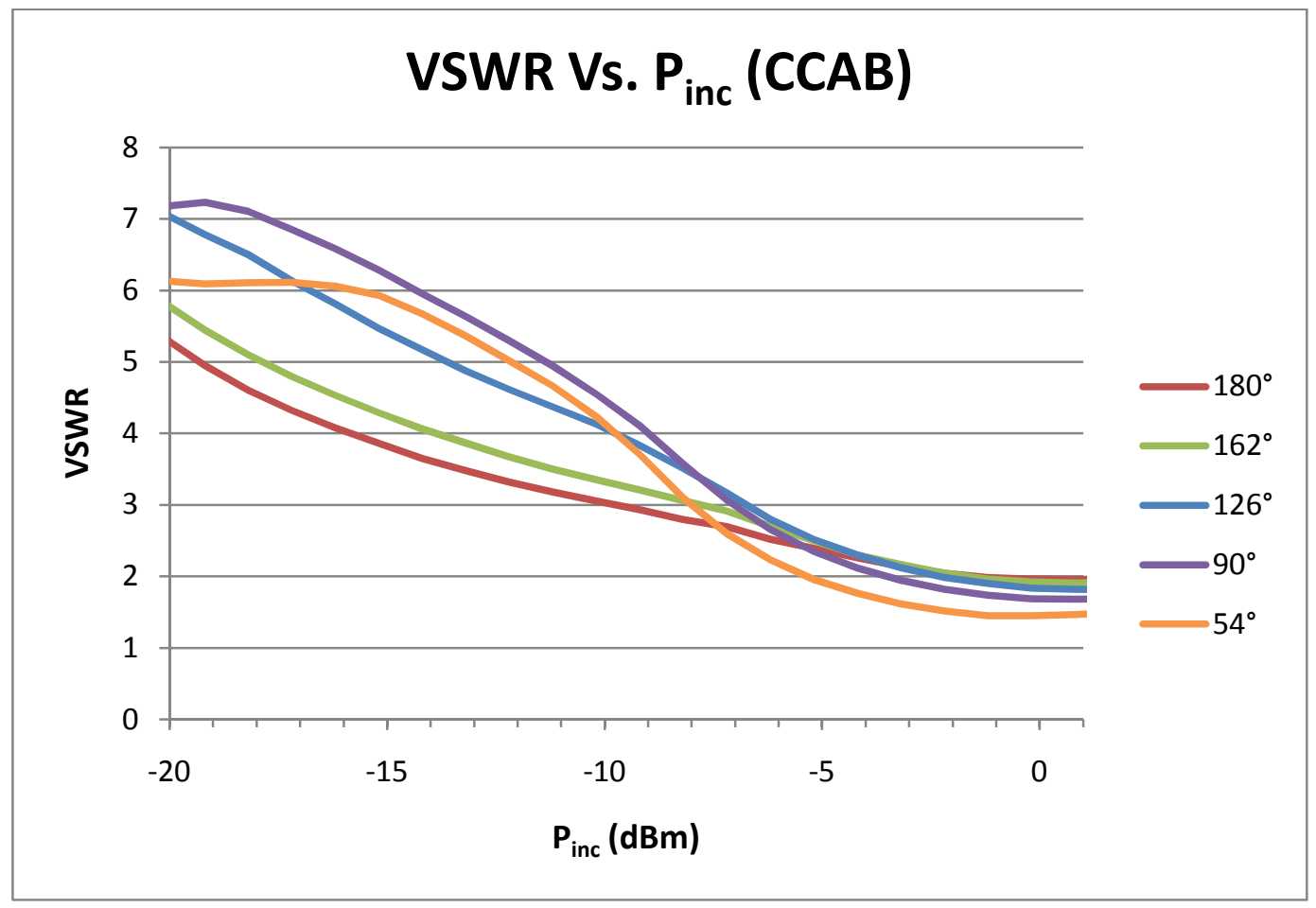

Figure 49: VSWR for CCAB amplifiers over $20 \mathrm{~dB}$ range 
The VSWR for the NB amplifiers fluctuates by approximately a factor of 2 within the $20 \mathrm{dBm}$ to $0 \mathrm{dBm}$ operating range except for at the $162^{\circ}$ degree configuration. This amplifier is biased at $0.03 \mathrm{~V}$. Referring to Figure 21, this bias point is close to the center of the biasing range where the input reactance greatly fluctuates. This might explain why the VSWR for this setting is dramatically poorer than the other NB amplifiers.

The VSWR for the CCAB amplifiers fluctuates by a factor of 3 to 4 for all the amplifier settings. The fluctuation is also rather gradual compared to the NB amplifiers. This is most likely because the CCAB amplifiers are dynamically controlling the bias point, which is having an effect on the input reactance. Also note the VSWR does plateau for each setting, at which point the bias point is most likely not changing significantly, since the input is so small.

Figure 50 and Figure 51 show the operational gain for the NB amplifiers. 


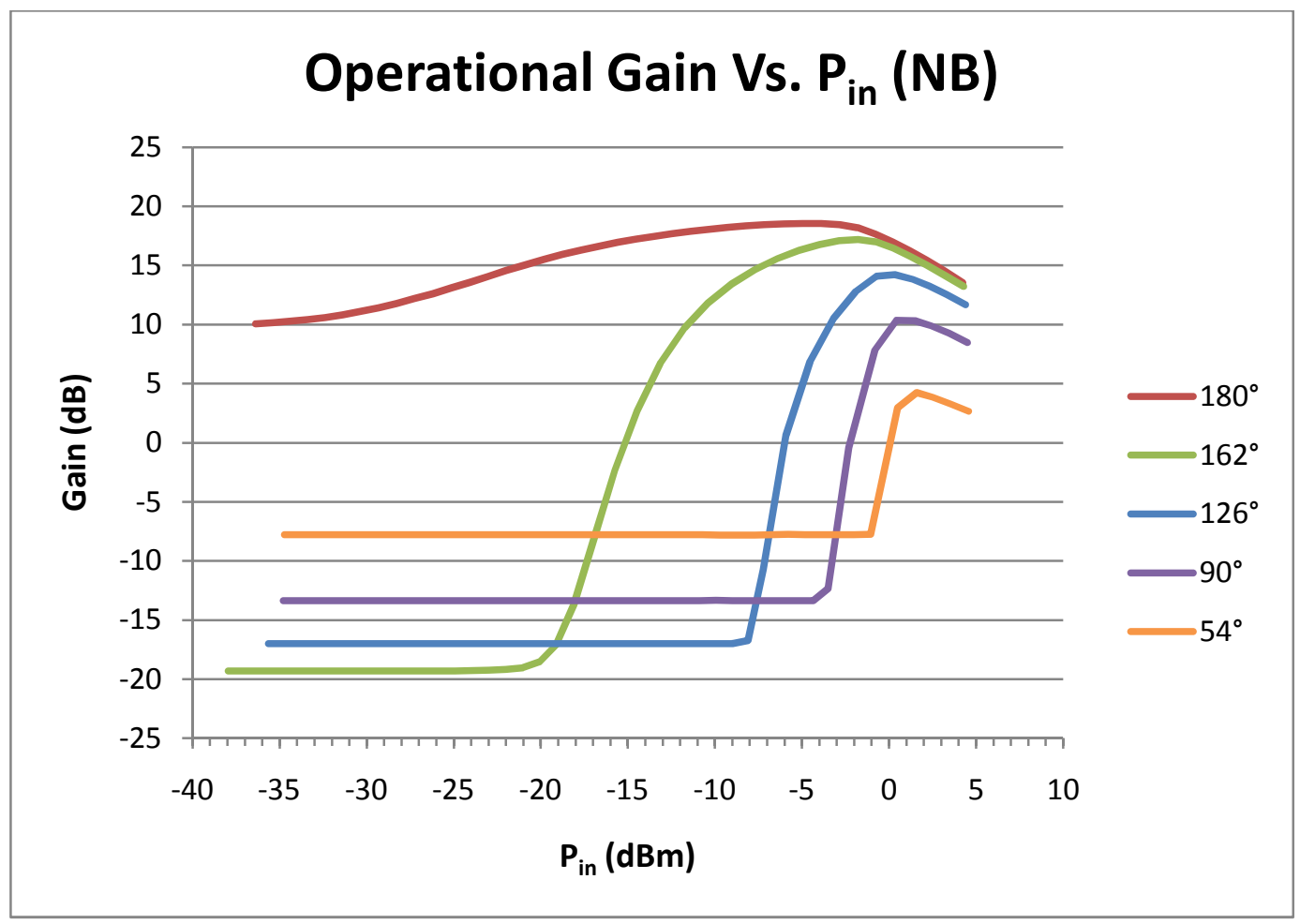

Figure 50: Operational gain for NB amplifiers over $40 \mathrm{~dB}$ range

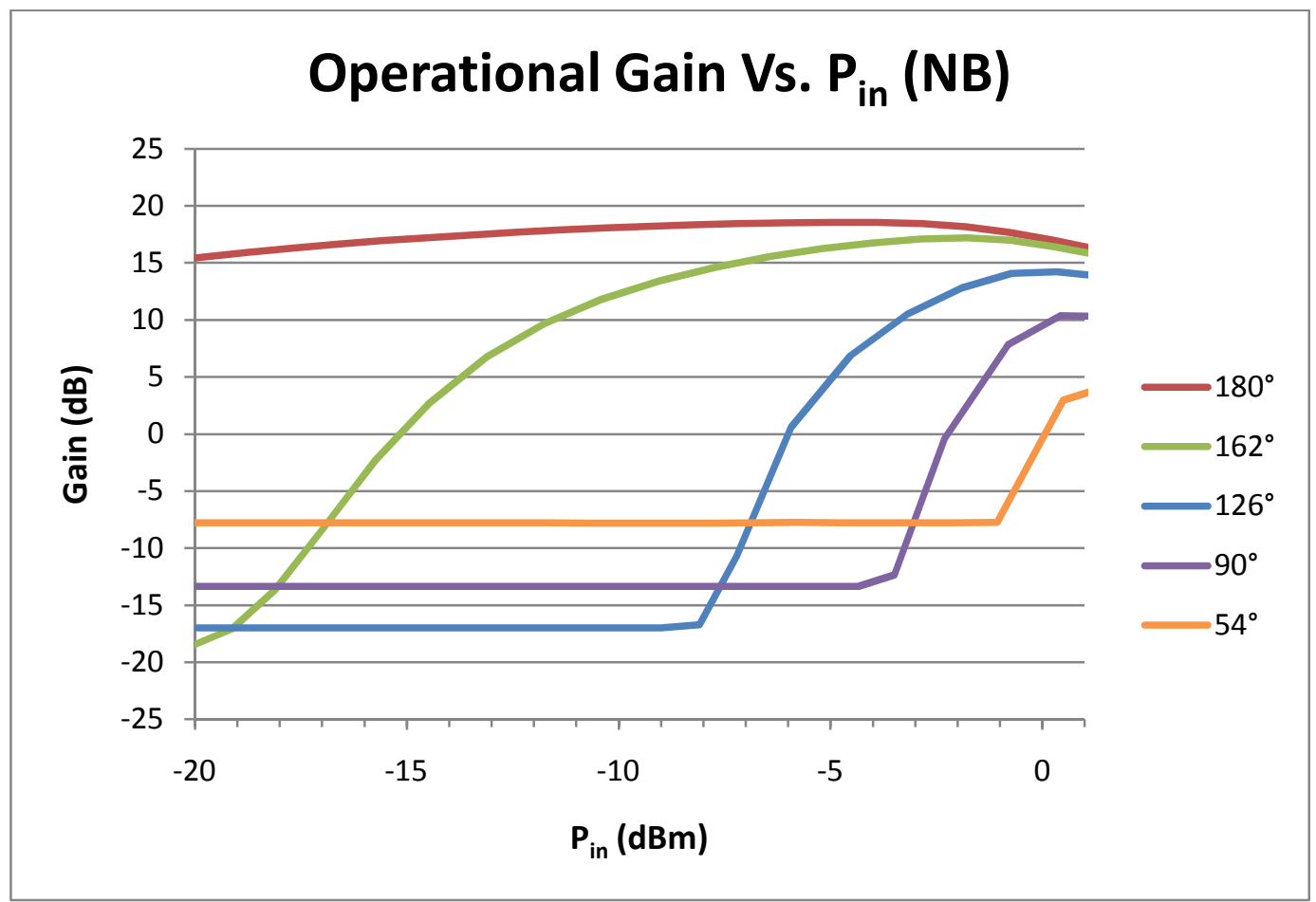

Figure 51: Operational gain for NB amplifiers over $20 \mathrm{~dB}$ range 
Figure 52 and Figure 53 show the operation gain for the CCAB amplifiers.

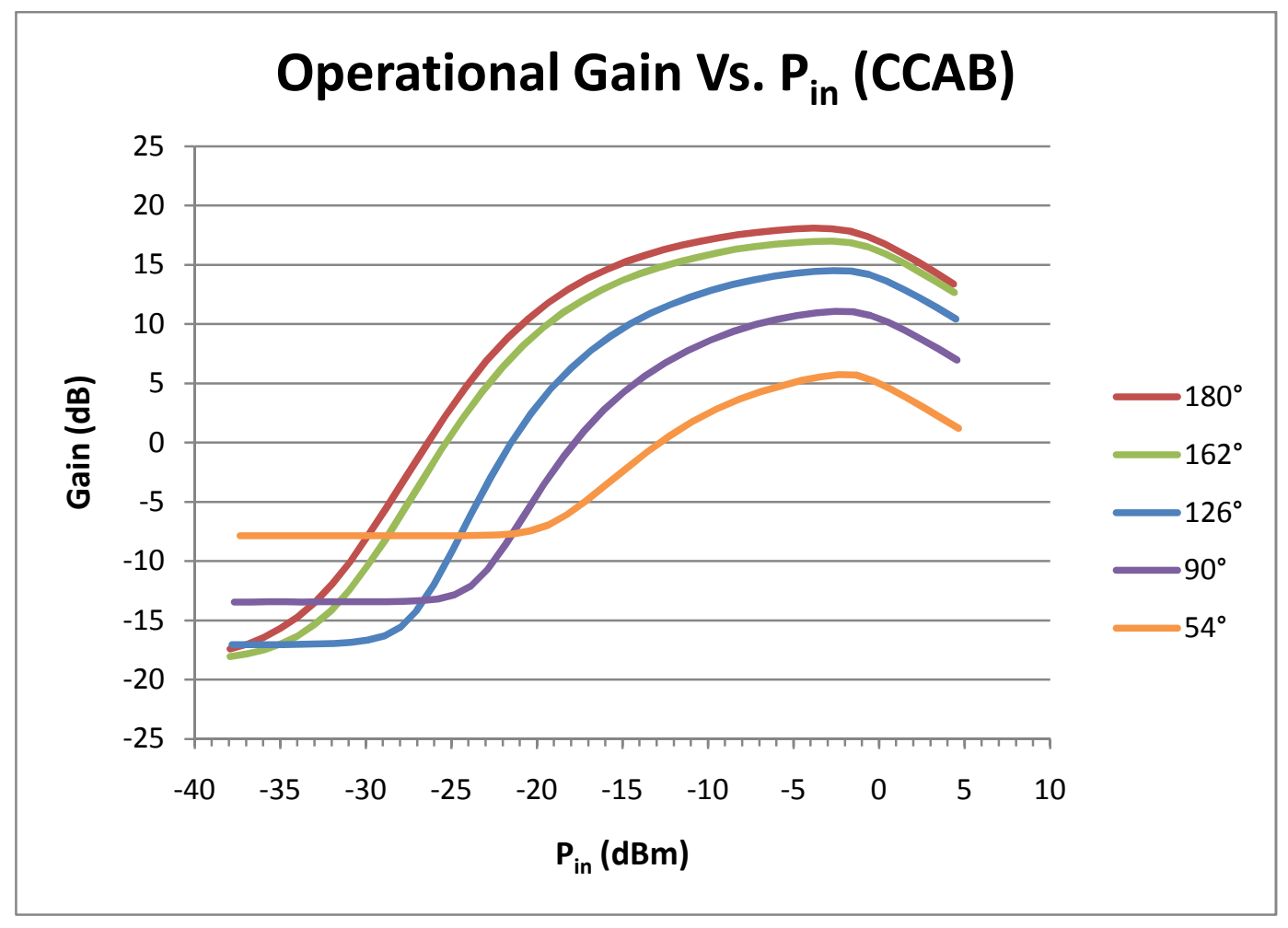

Figure 52: Operational gain for $C C A B$ amplifiers over $40 \mathrm{~dB}$ range 


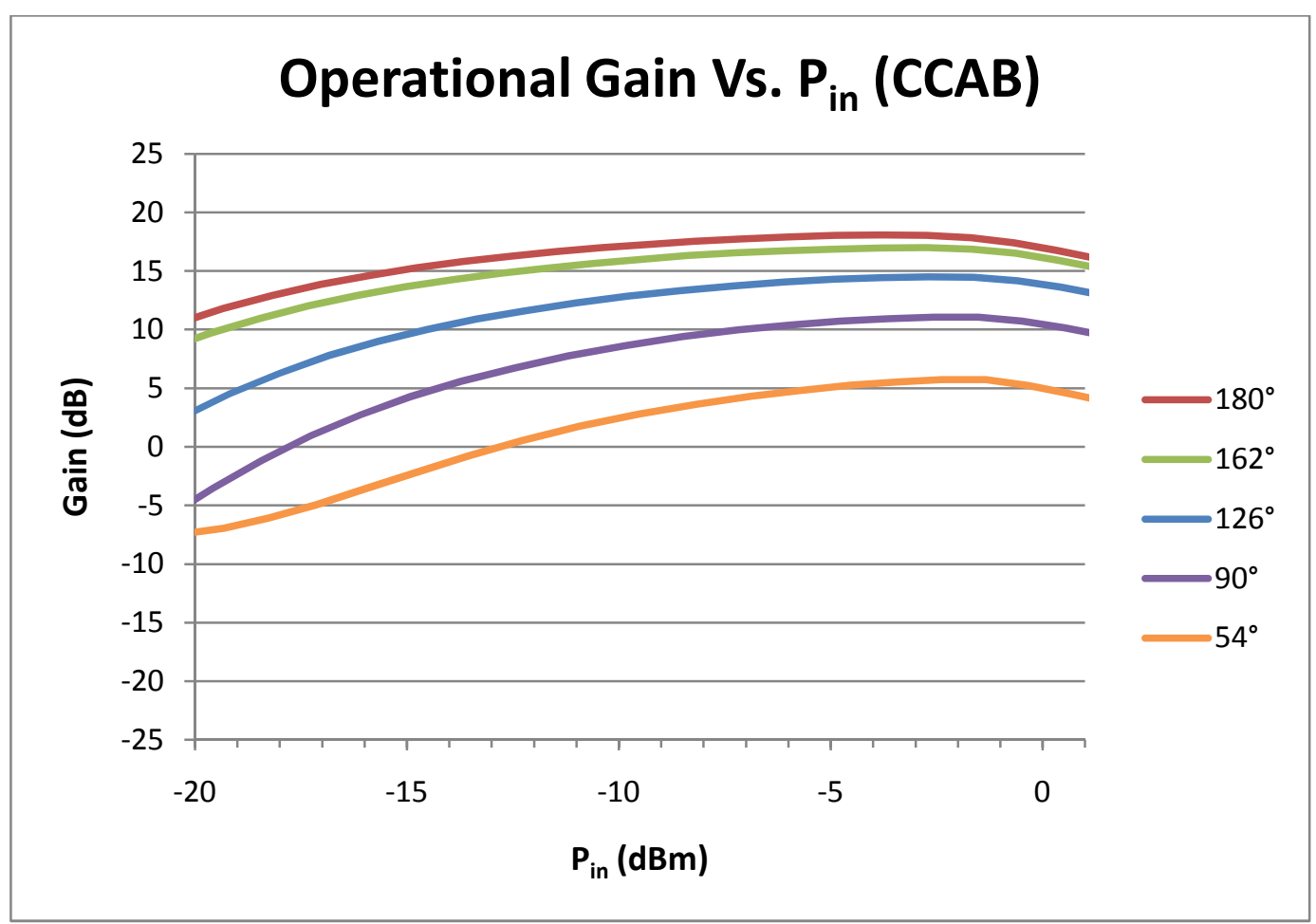

Figure 53: Operational gain for CCAB amplifiers over $20 \mathrm{~dB}$ range

The operational gain for the CCAB amplifiers shows that the biasing scheme for the amplifiers actually keeps the gain fluctuation down even less than the transducer gain suggested. But the fact that the VSWR fluctuates with bias results in addition fluctuation for transducer gain.

Figure 54 and Figure 55 show the drain efficiency for the NB amplifiers. 


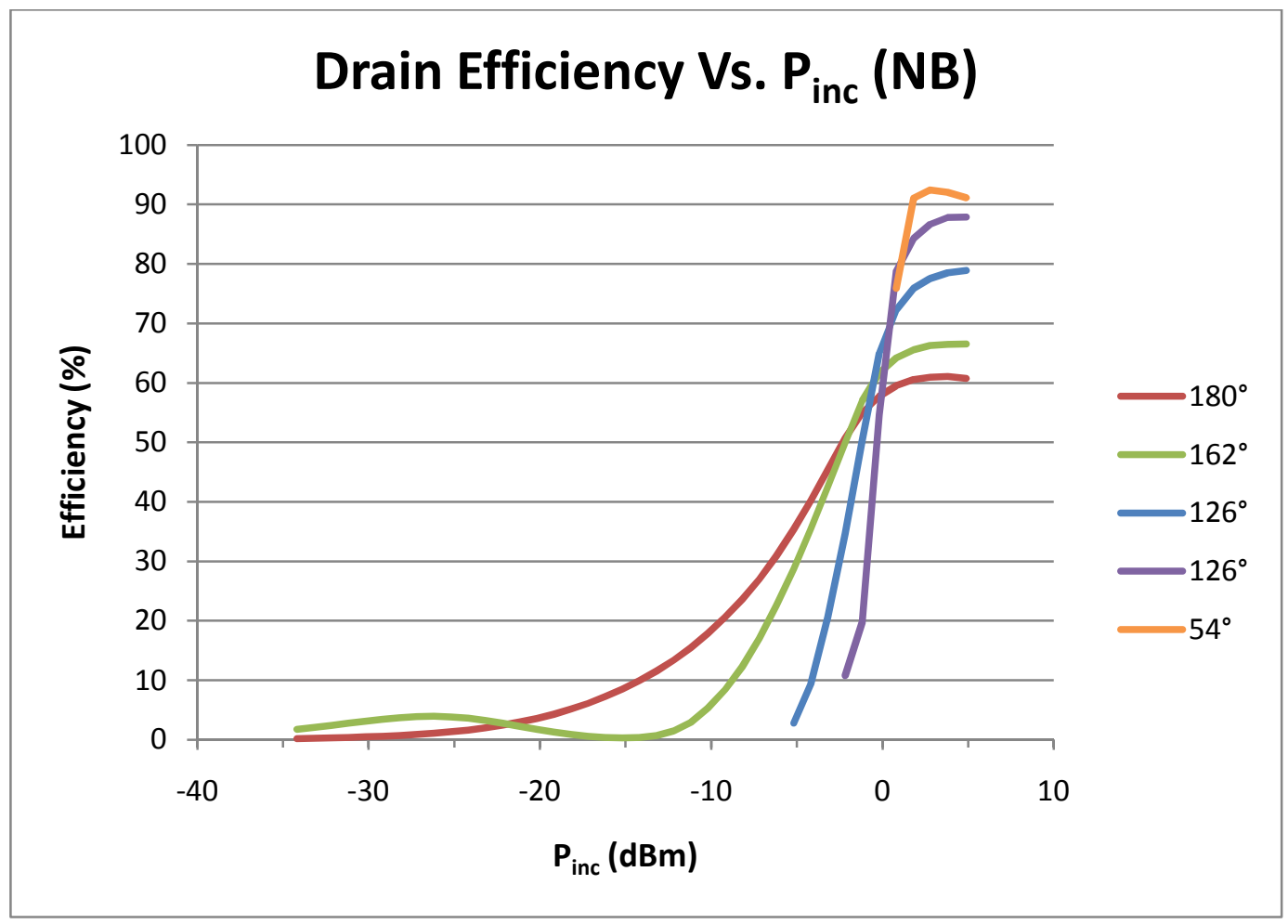

Figure 54: Drain efficiency for NB amplifiers over $40 \mathrm{~dB}$ range

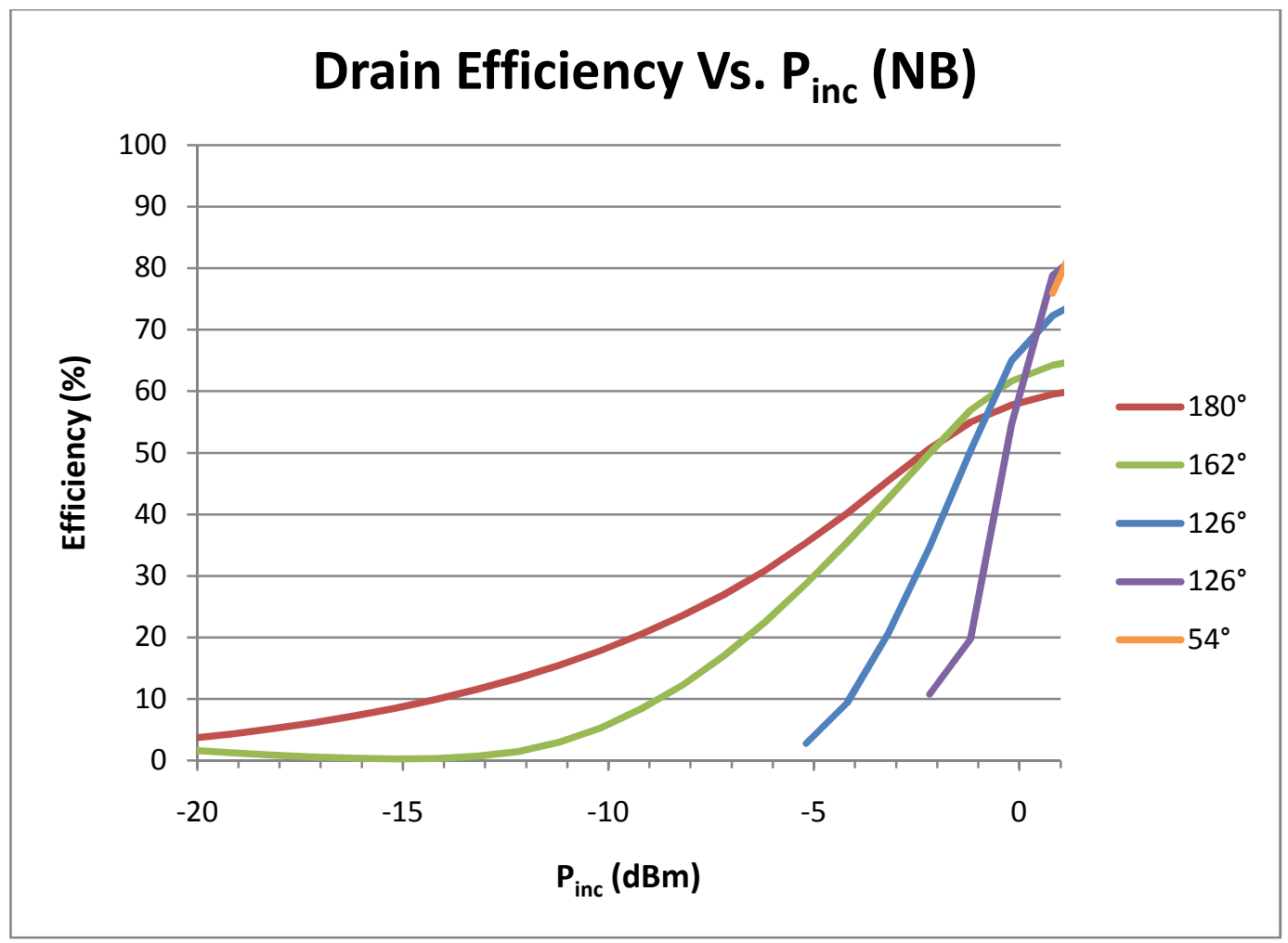

Figure 55: Drain efficiency for NB amplifiers over $20 \mathrm{~dB}$ range 
Figure 56 and Figure 57 show the drain efficiency for the $C C A B$ amplifiers

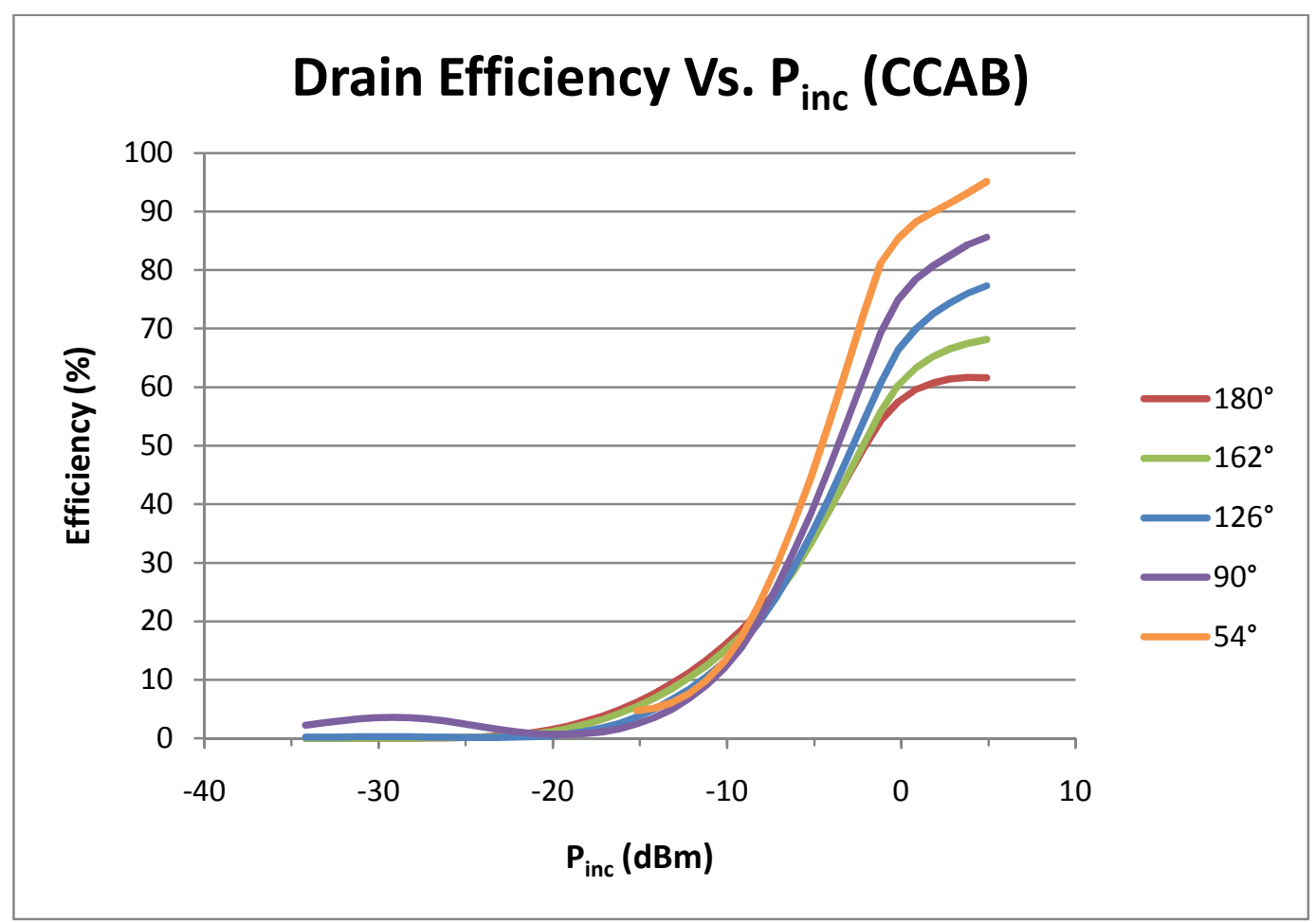

Figure 56: Drain efficiency for CCAB amplifiers over $40 \mathrm{~dB}$ range 


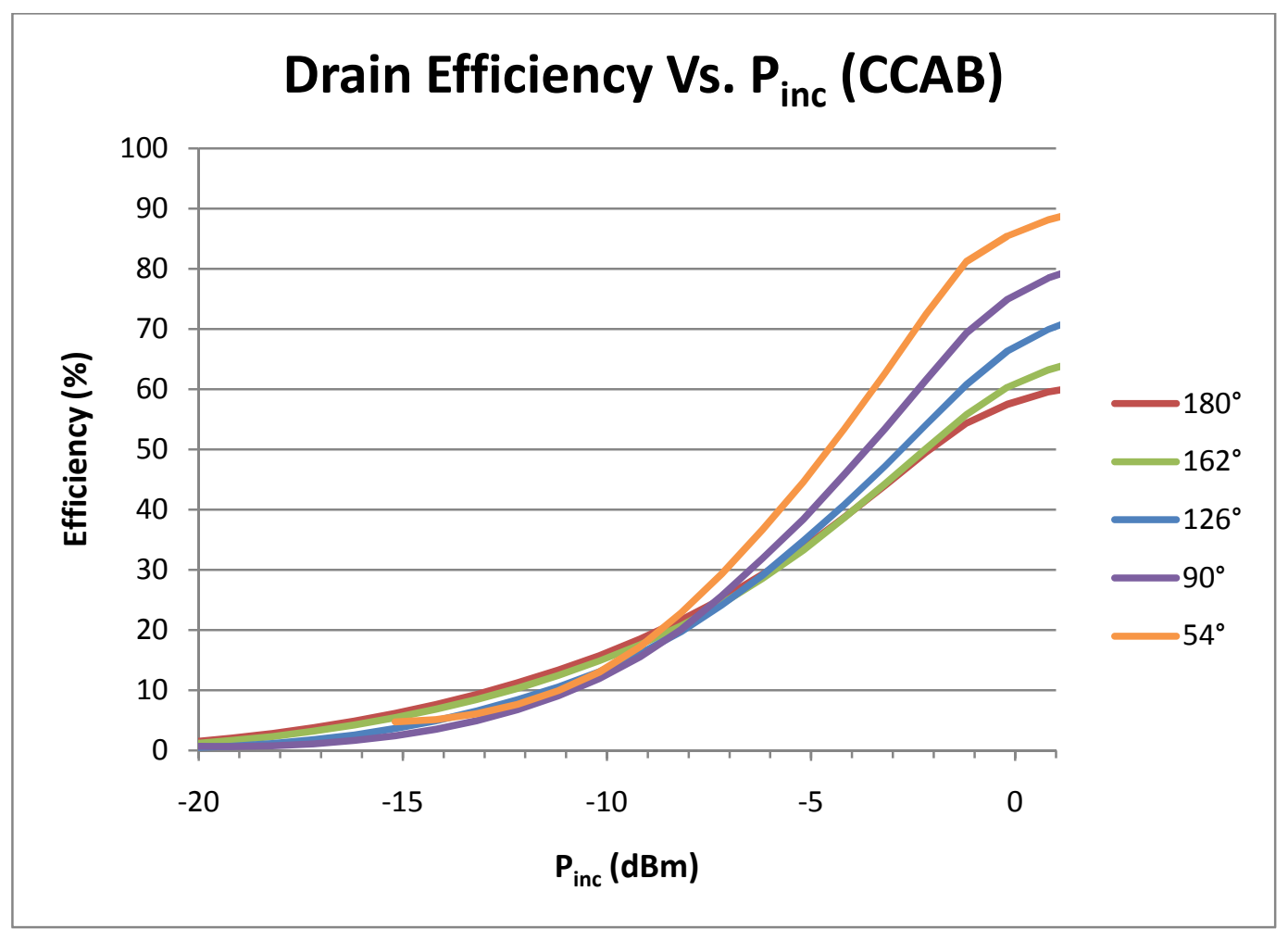

Figure 57: Drain efficiency for CCAB amplifiers over $20 \mathrm{~dB}$ range

The peak drain efficiency for the CCAB amplifiers and NB amplifiers differs by less than $\pm 4 \%$ for each angle setting. The system efficiency of the CCAB amplifiers will be degraded by the power required for the op-amps and biasing transistors but should still should still offer improvements in efficiency over class B operation. 
Figure 58 shows the $A C$ drain voltage as a function of the $A C$ gate voltage for the NB amplifiers.

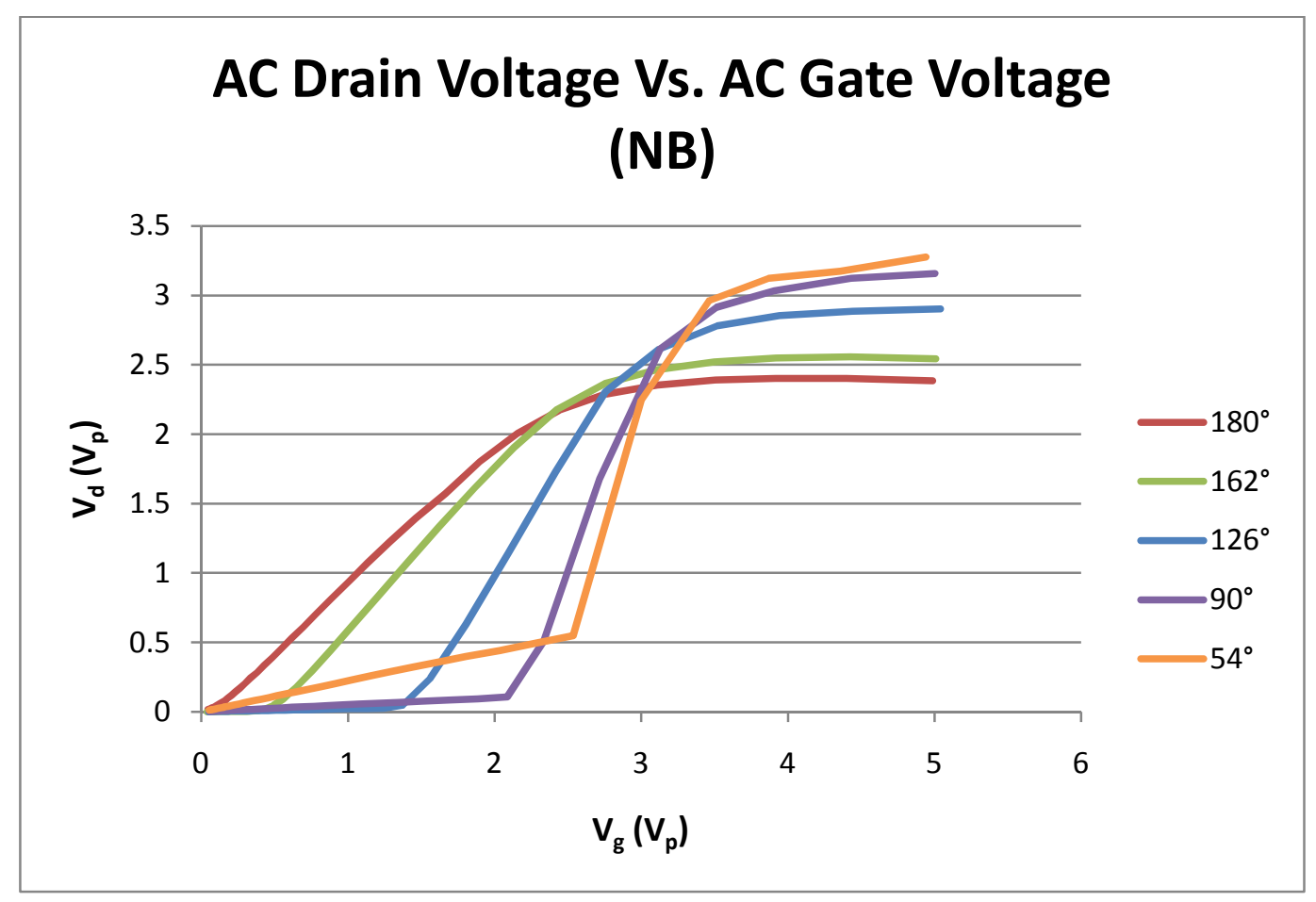

Figure 58: AC drain voltage for NB amplifiers 
Figure 59 shows the $A C$ drain voltage as a function of the $A C$ gate voltage for the CCAB amplifiers.

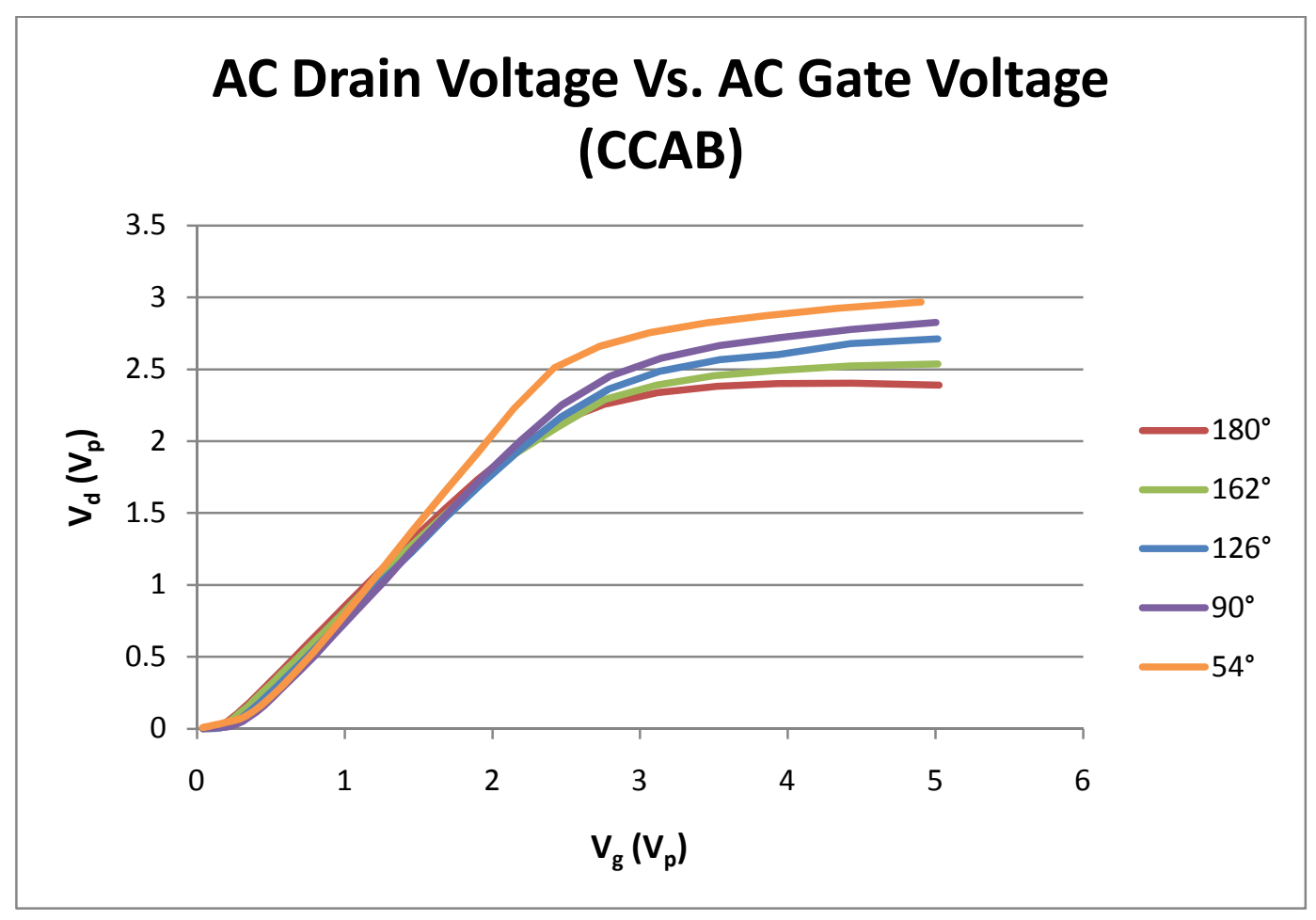

Figure 59: $A C$ drain voltage for $C C A B$ amplifiers 
Figure 60 shows the Class $C$ bias voltage as a function of the $A C$ gate voltage for the CCAB amplifiers.

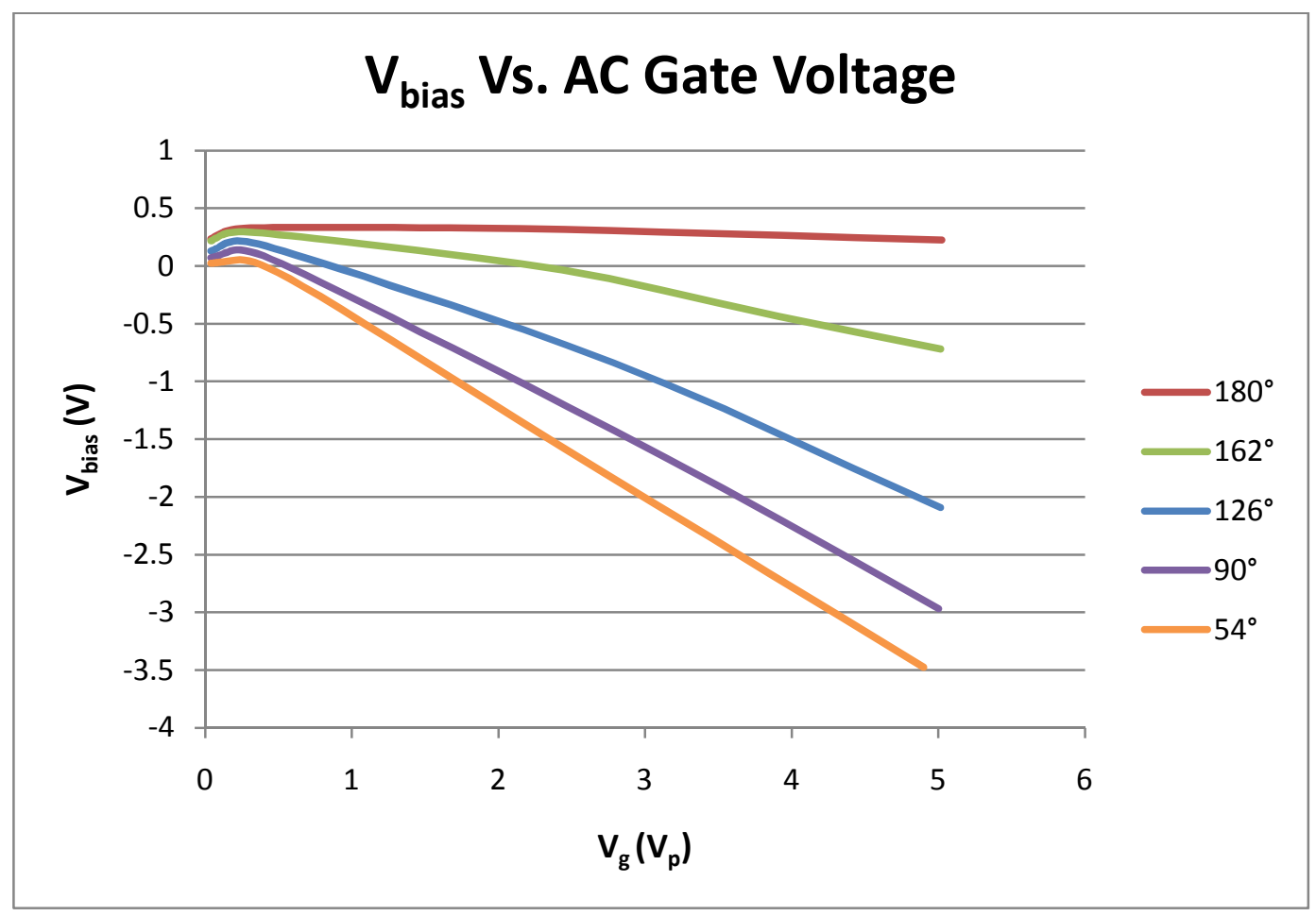

Figure 60: Class $C$ bias voltage for CCAB amplifiers 
Figure 61 shows the Class $\mathrm{C}$ bias for values of $\mathrm{K}$ at $0.2,0.7,2.0$, 3.2, 5.0 , and 6.75 for the $126^{\circ}$ amplifier.

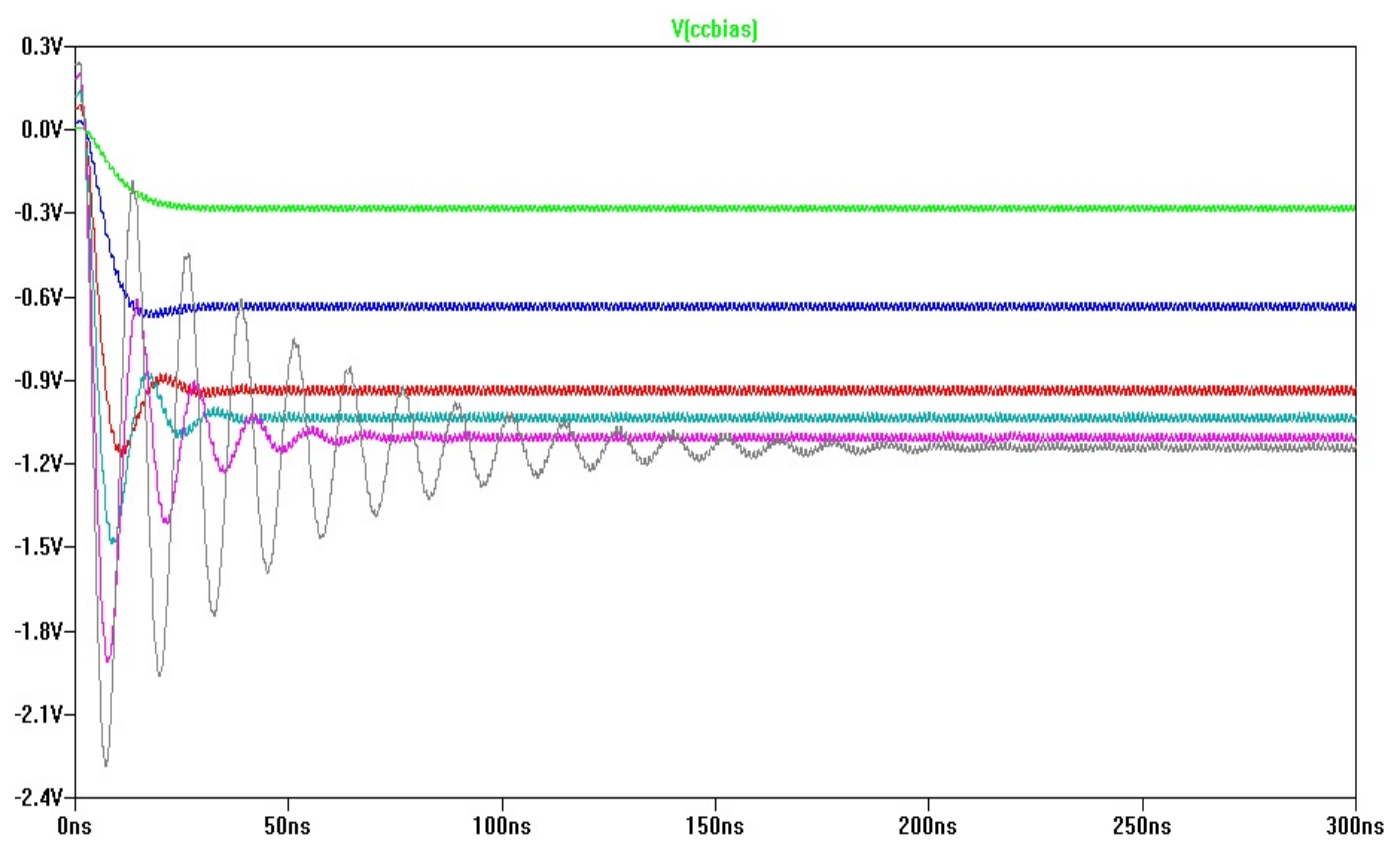

Figure 61: Class $C$ bias voltage at $126^{\circ}$ setting for multiple values of $K$ 
Figure 62 shows the conduction angle as a function of incident power for multiple values of $\mathrm{K}$ in the $126^{\circ} \mathrm{CCAB}$ ampl ifier

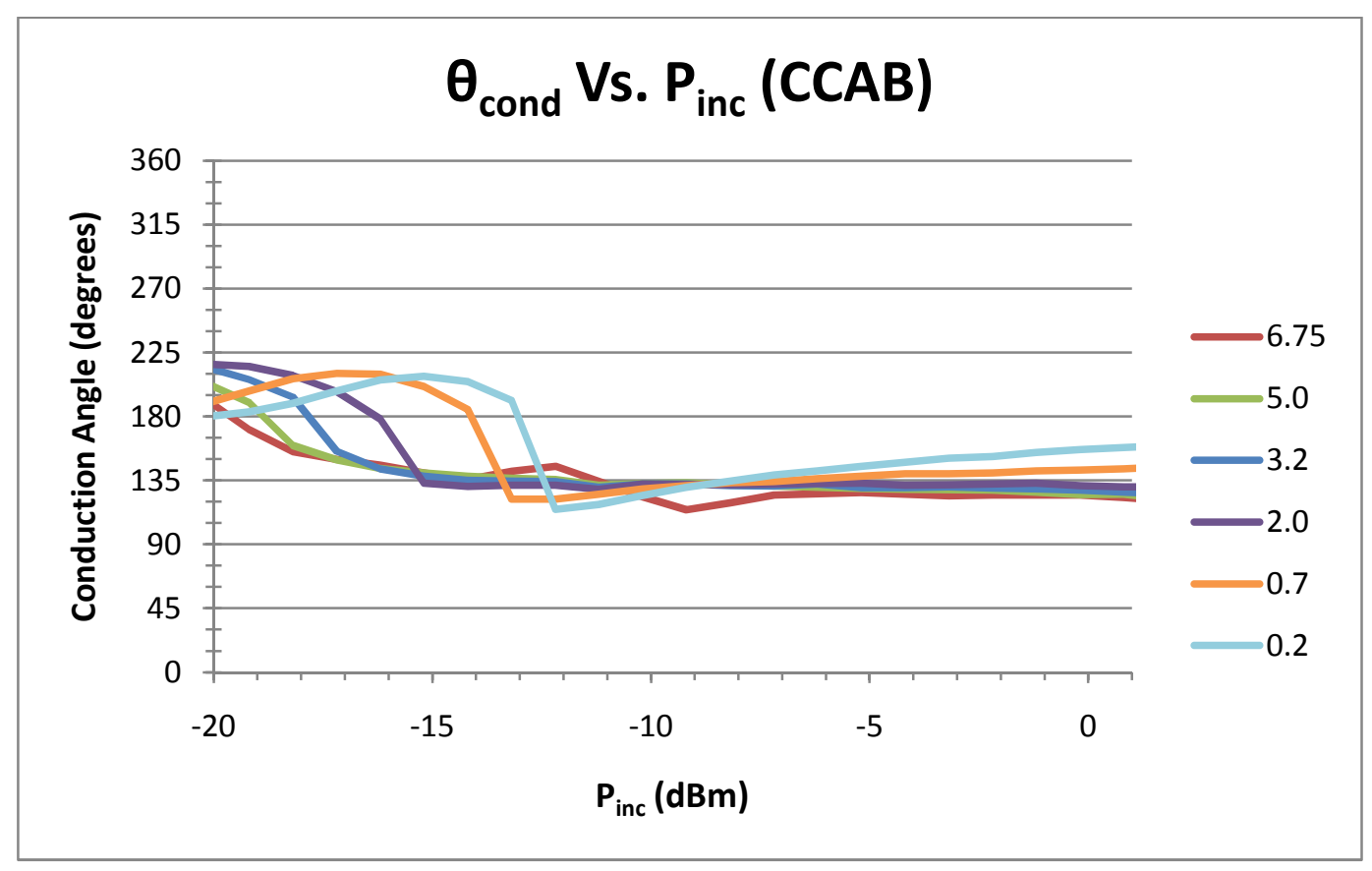

Figure 62: Conduction angle of $126^{\circ} \mathrm{CCAB}$ amplifier for multiple values of $\mathrm{K}$ 
Figure 63 shows the transducer gain as a function of incident power for multiple values of $\mathrm{K}$ in the $126^{\circ} \mathrm{CCAB}$ amplifier

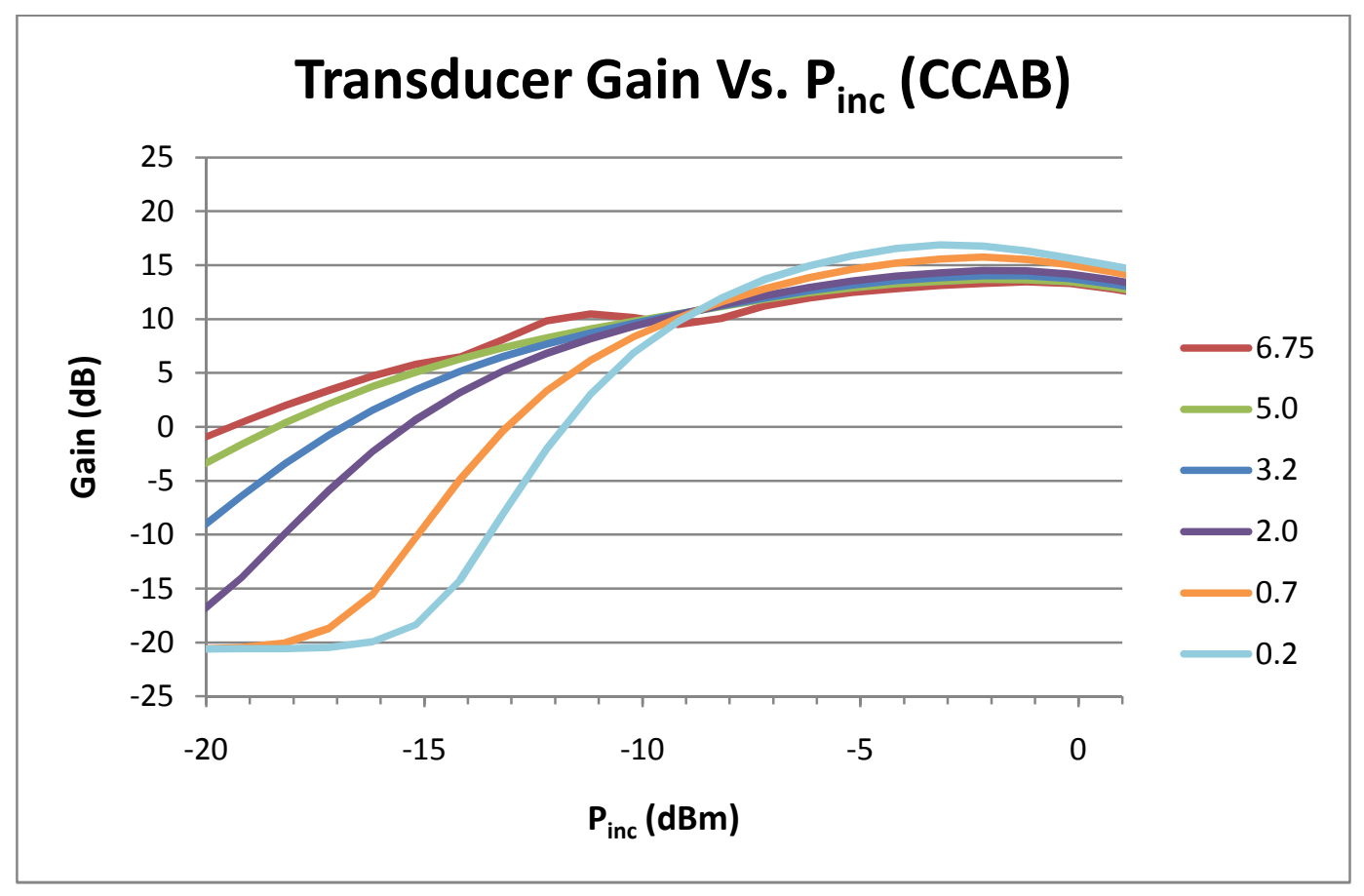

Figure 63: Transducer gain of $126^{\circ} \mathrm{CCAB}$ amplifier for multiple values of $\mathrm{K}$ 
Figure 64 shows the operational gain as a function of input power for multiple values of $\mathrm{K}$ in the $126^{\circ} \mathrm{CCAB}$ amplifier

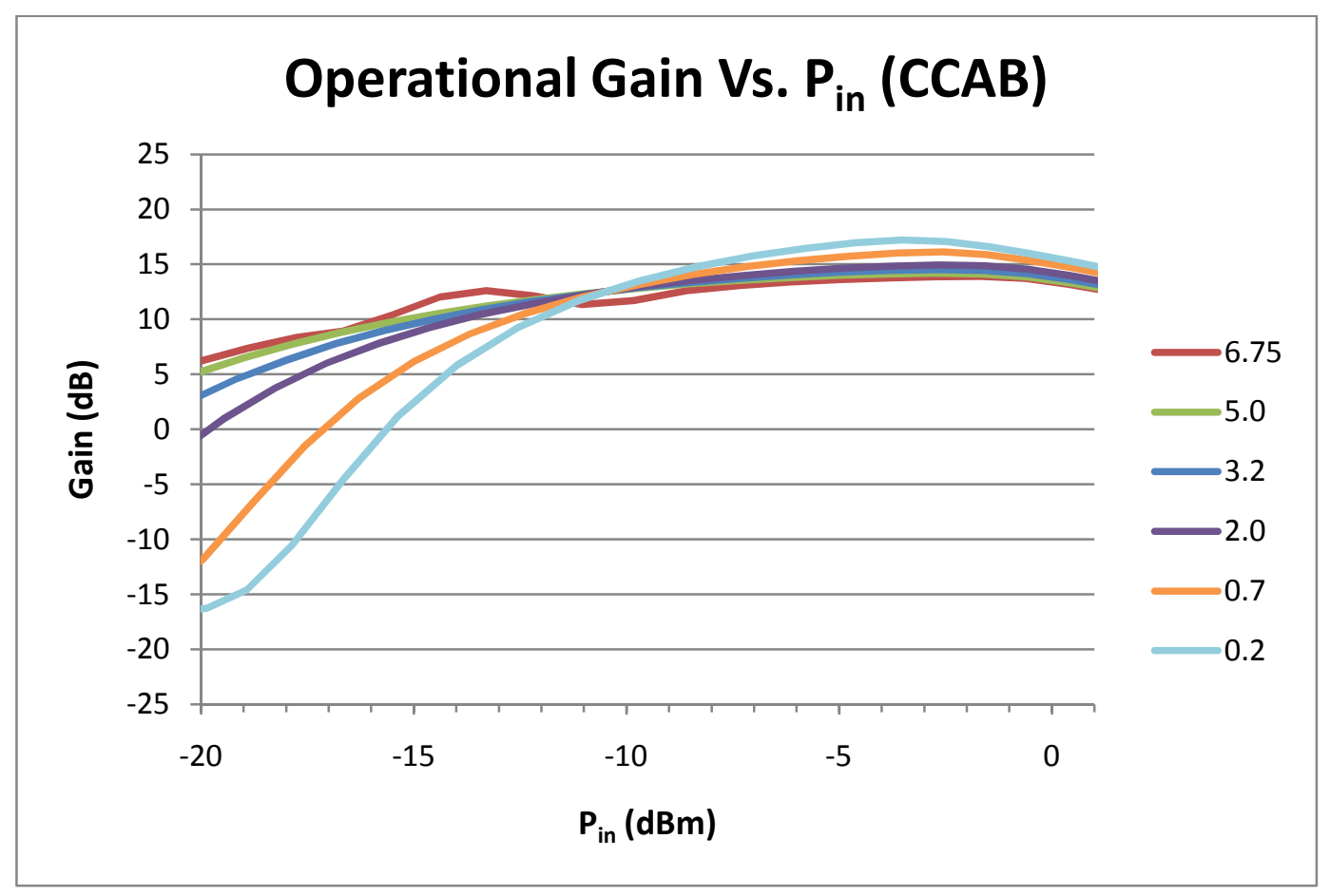

Figure 64: Operational gain of $126^{\circ} \mathrm{CCAB}$ amplifier for multiple values of $\mathrm{K}$

Increases in $\mathrm{K}$ improve the linearity of the amplifier. However, If the value of $\mathrm{K}$ must remain low enough so that the settling time of the bias voltage does not affect the bandwidth. Increasing the value of $\mathrm{K}$ also imposes the risk of the amplifier becoming unstable. 
Figure 65 shows the conduction angle as a function of incident power for multiple values of temperature in the $126^{\circ} \mathrm{CCAB}$ amplifier

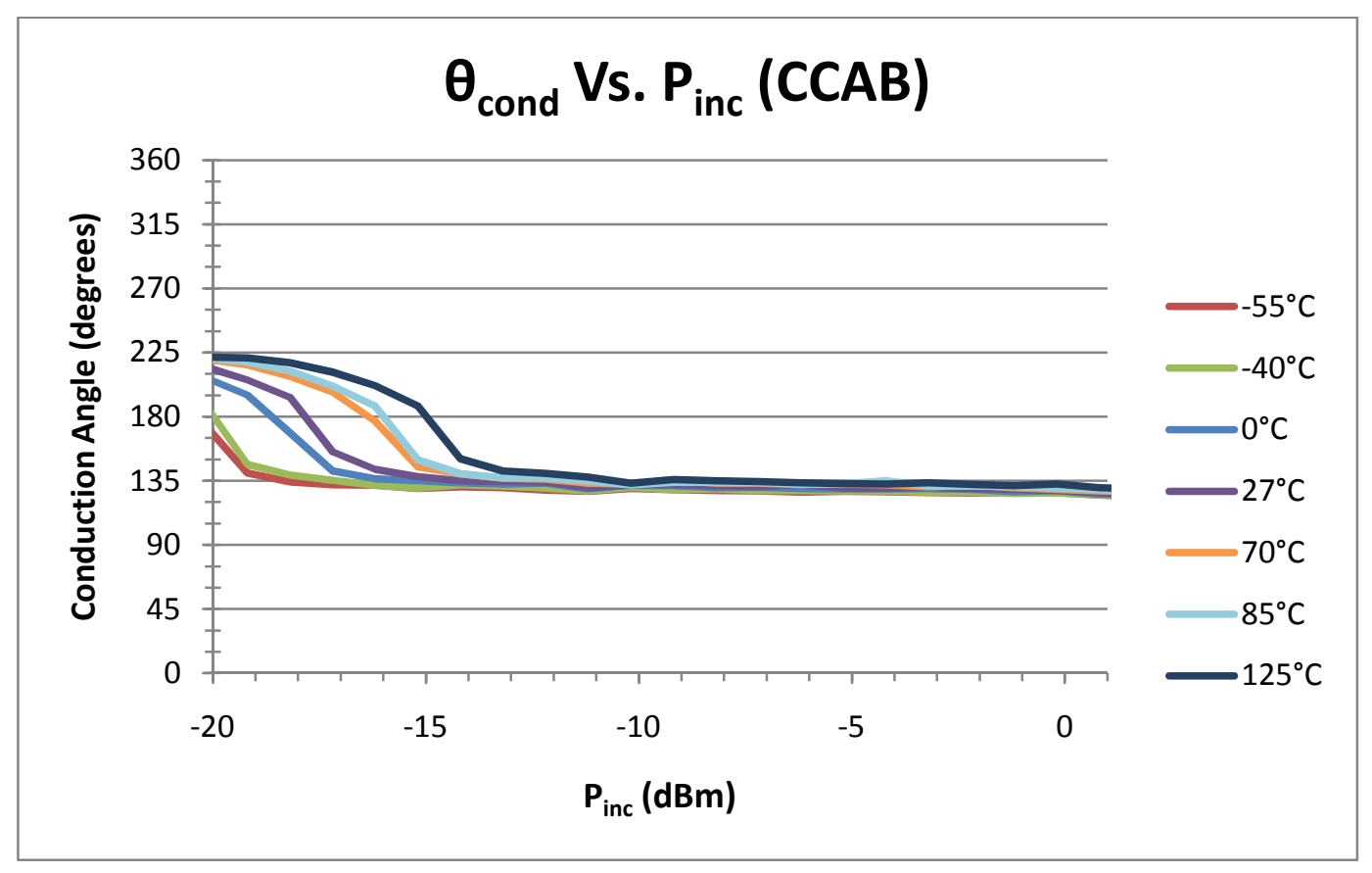

Figure 65: Conduction angle of $126^{\circ} \mathrm{CCAB}$ amplifier for multiple temperatures 
Figure 66 shows the transducer gain as a function of incident power for multiple values of temperature in the $126^{\circ} \mathrm{CCAB}$ amplifier

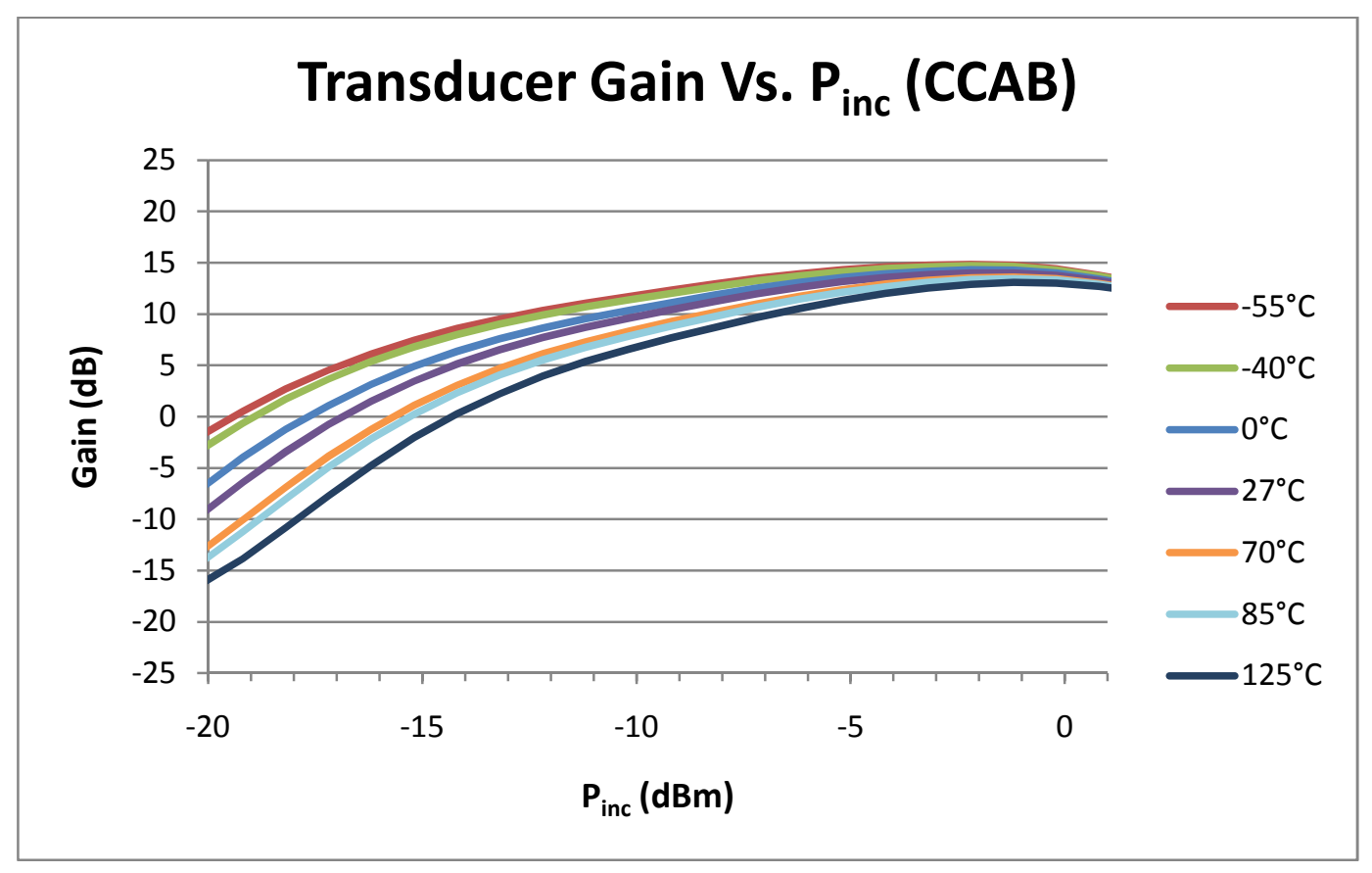

Figure 66: Transducer gain of $126^{\circ} \mathrm{CCAB}$ amplifier $f$ or multiple temperatures 
Figure 67 shows the operational gain as a function of incident power for multiple values of temperature in the $126^{\circ} \mathrm{CCAB}$ amplifier

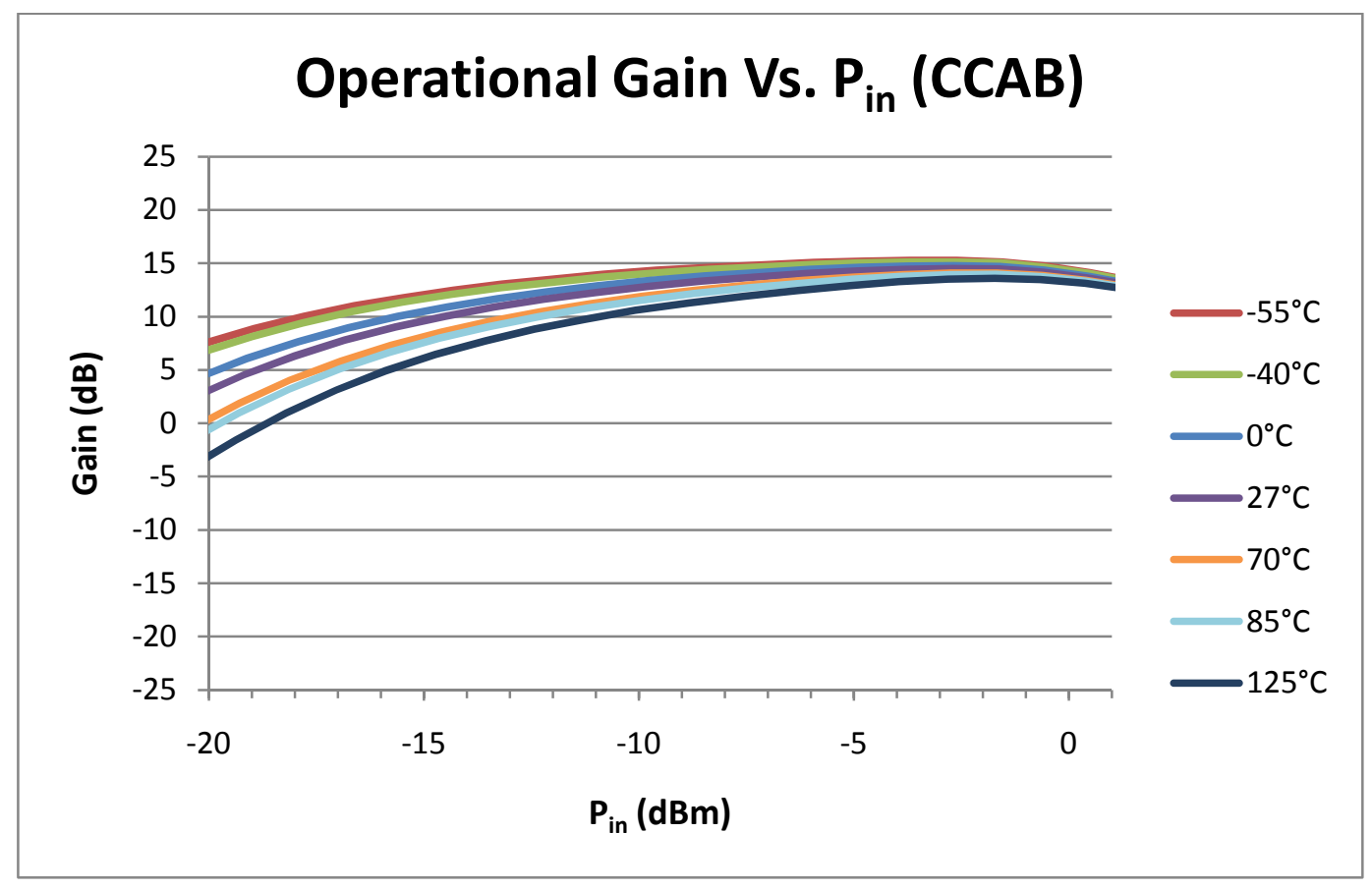

Figure 67: Operational gain of $126^{\circ} \mathrm{CCAB}$ amplifier for multiple temperatures

Changes in temperature cause variation in the gain of the amplifier. These changes in gain become more pronounced as the input power is decreased.

Figure 68 and Figure 69 show the conduction angle as a function of frequency for multiple values of $\mathrm{P}_{\mathrm{inc}}$ in the $126^{\circ} \mathrm{CCAB}$ amplifier 


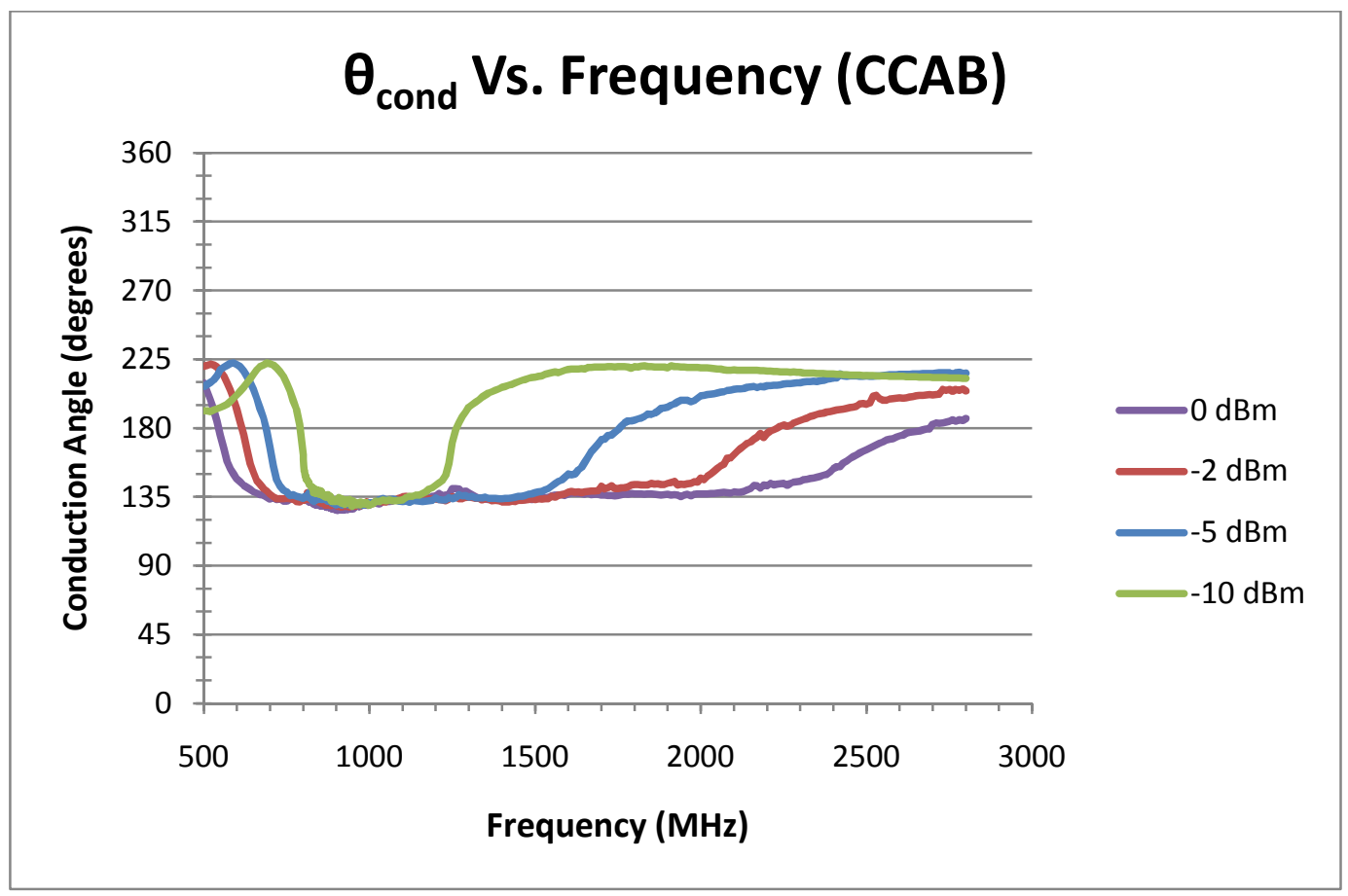

Figure 68: Conduction angle of $126^{\circ} \mathrm{CCAB}$ amplifier for multiple values of $\mathrm{P}_{\mathrm{inc}}$ over $2.3 \mathrm{GHz}$ range

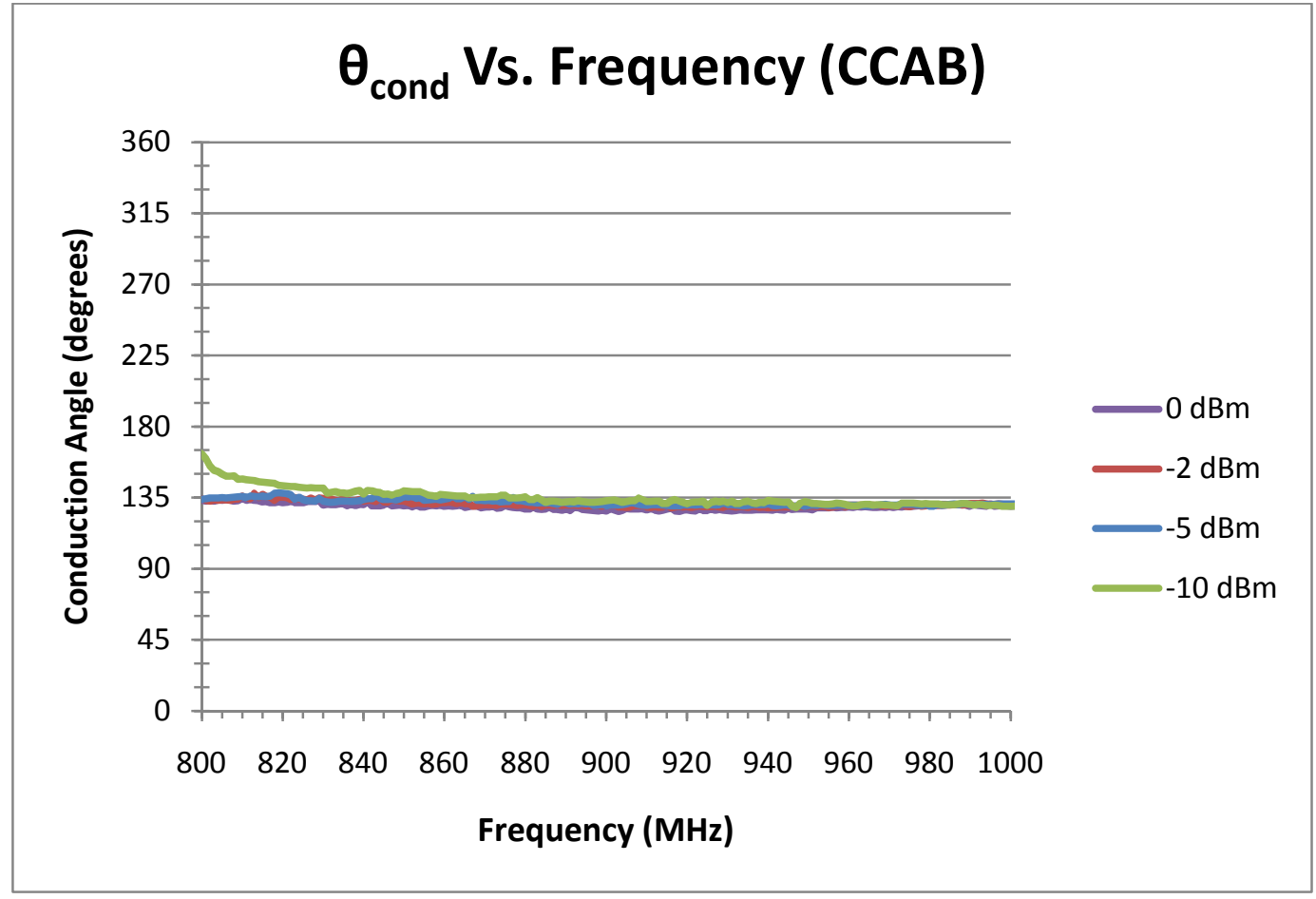

Figure 69: Conduction angle of $126^{\circ} \mathrm{CCAB}$ amplifier for multiple values of $\mathrm{P}_{\text {inc }}$ over $200 \mathrm{MHz}$ range 
Figure 70 and Figure 71 show the transducer gain as a function of frequency for multiple values of $\mathrm{P}_{\text {inc }}$ in the $126^{\circ} \mathrm{CCAB}$ amplifier

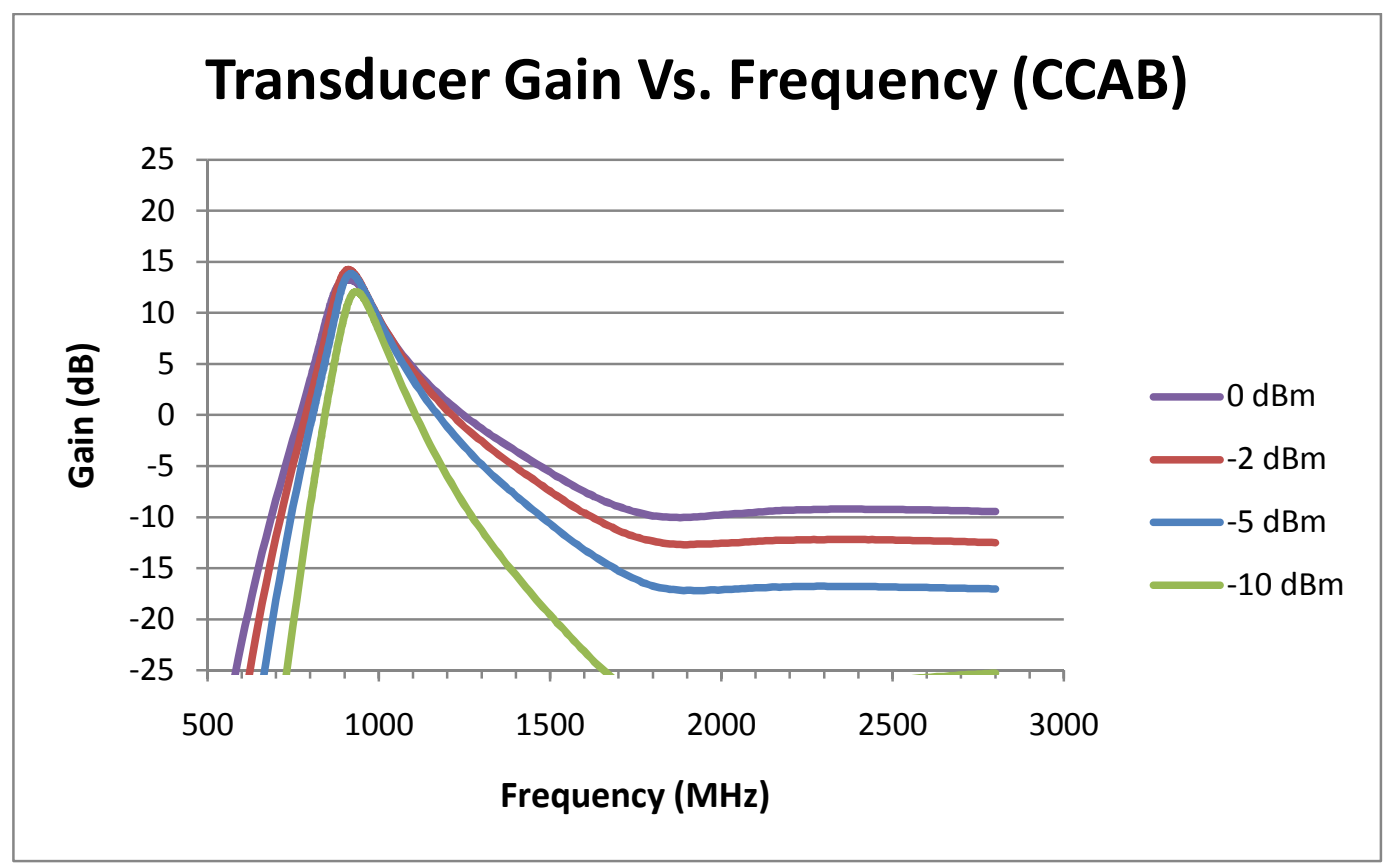

Figure 70: Transducer gain of $126^{\circ} \mathrm{CCAB}$ amplifier $f$ or multiple values of $\mathrm{P}_{\mathrm{inc}}$ over $2.3 \mathrm{GHz}$ range 


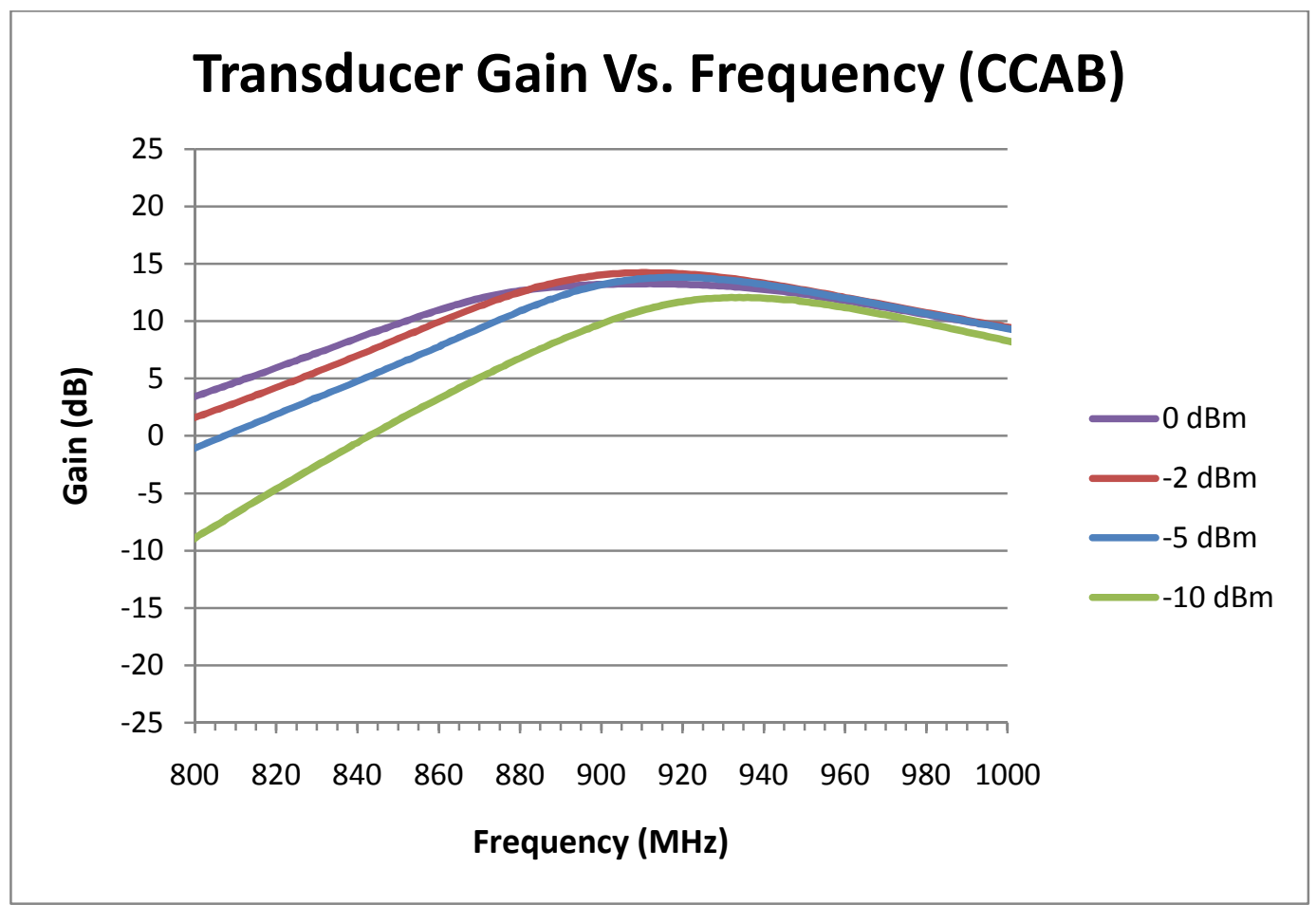

Figure 71: Transducer gain of $126^{\circ} \mathrm{CCAB}$ amplifier for multiple values of $\mathrm{P}_{\text {inc }}$ over $200 \mathrm{MHz}$ range 
Figure 72 shows the VSWR as a function of frequency for multiple values of $P_{\text {inc }}$ in the $126^{\circ} \mathrm{CCAB}$ amplifier

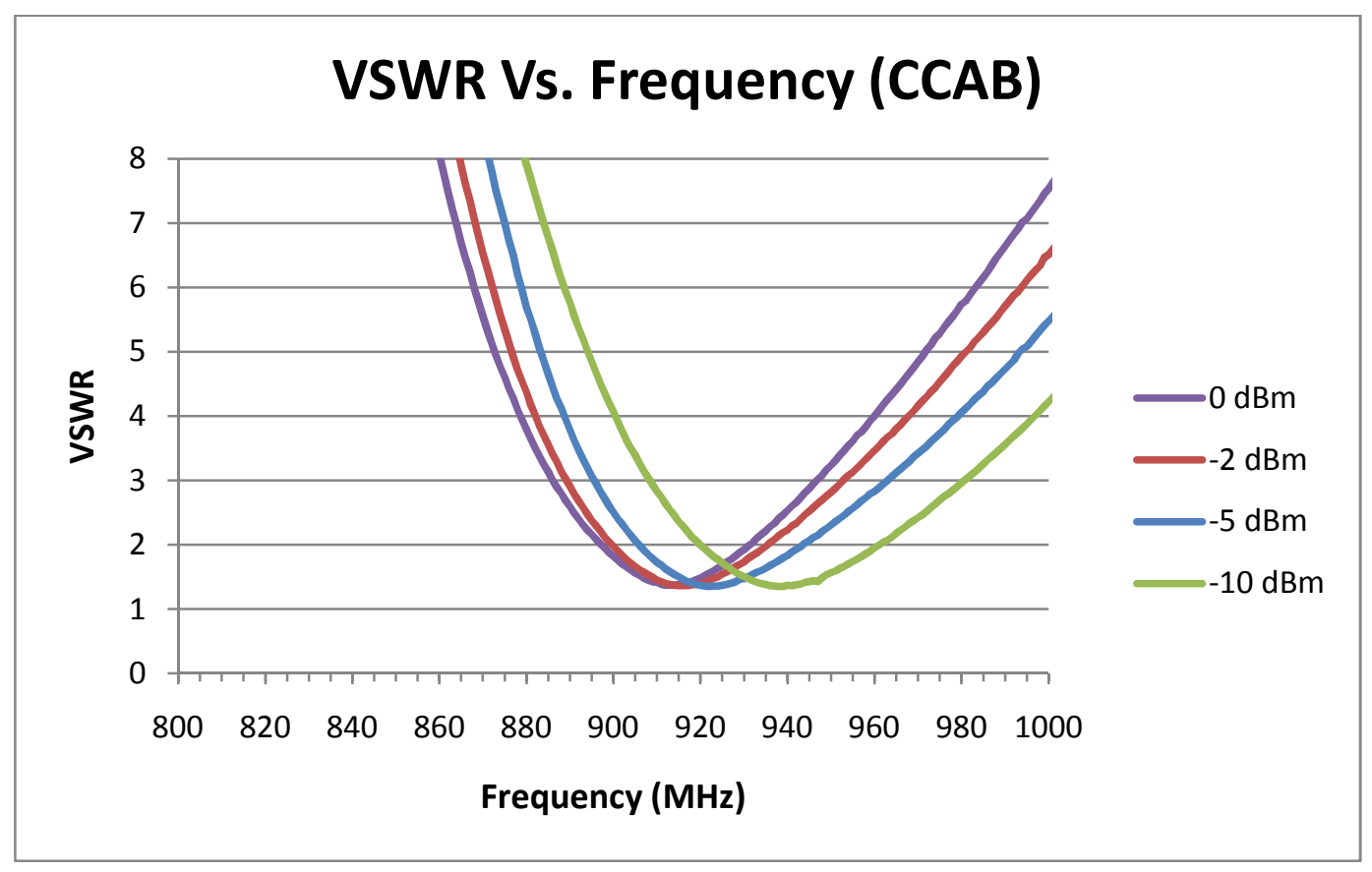

Figure 72: VSWR of $126^{\circ} \mathrm{CCAB}$ amplifier for multiple values of $\mathrm{P}_{\text {inc }}$ over $200 \mathrm{MHz}$ range

Changes in frequency do not significantly affect the biasing circuitry. However, they do affect the input network, which changes the amount of power that is input into the amplifier. This causes a change in conduction angle once the input power drops below the effective operating range. 
An input source composed of $\frac{V_{a m p}}{2}(\cos (2 \pi \cdot 910 \mathrm{MHz} \cdot t)+$ $\cos (2 \pi \cdot 890 \mathrm{MHz} \cdot t))$ was used to created an $\mathrm{AM}$ modulated $900 \mathrm{MHz}$ input with $20 \mathrm{MHz}$ bandwidth.

Simulations with $\mathrm{V}_{\mathrm{amp}}$ set at $0.7 \mathrm{~V}$ were run. An FFT of the output power was taken. Figure 73 shows the power of each frequency component for the Class $\mathrm{B}, 126^{\circ} \mathrm{Class} \mathrm{C}$, and $126^{\circ} \mathrm{CCAB}$ amplifiers.

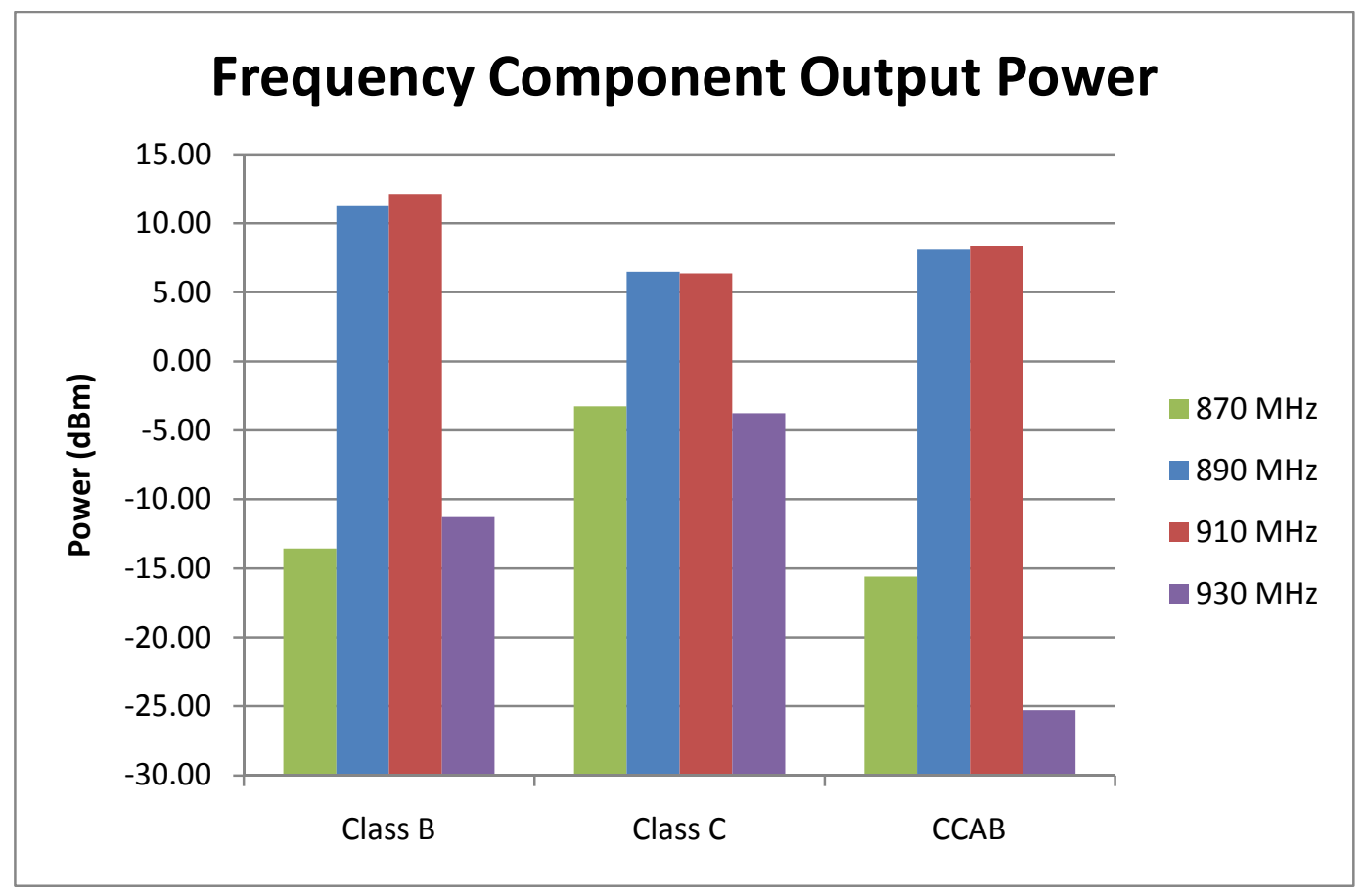

Figure 73: Output power of fundamental and $3^{\text {rd }}$ order intermodulation products for $0.7 \mathrm{~V}$ AM input 
Simulations with $\mathrm{V}_{\mathrm{amp}}$ set at $0.2 \mathrm{~V}, 0.35 \mathrm{~V}, 0.5 \mathrm{~V}$, were run as well. The power, in $\mathrm{dB}$, at the output for the $910 \mathrm{MHz}$ and $890 \mathrm{MHz}$ peaks were averaged. The power, in $\mathrm{dB}$, at the output for the $930 \mathrm{MHz}$ and $870 \mathrm{MHz}$ peak were also averaged. Figure 74 shows the difference between these two averages for the Class $B, 126^{\circ}$ Class $C$, and $126^{\circ} \mathrm{CC} A B$ amplifiers.

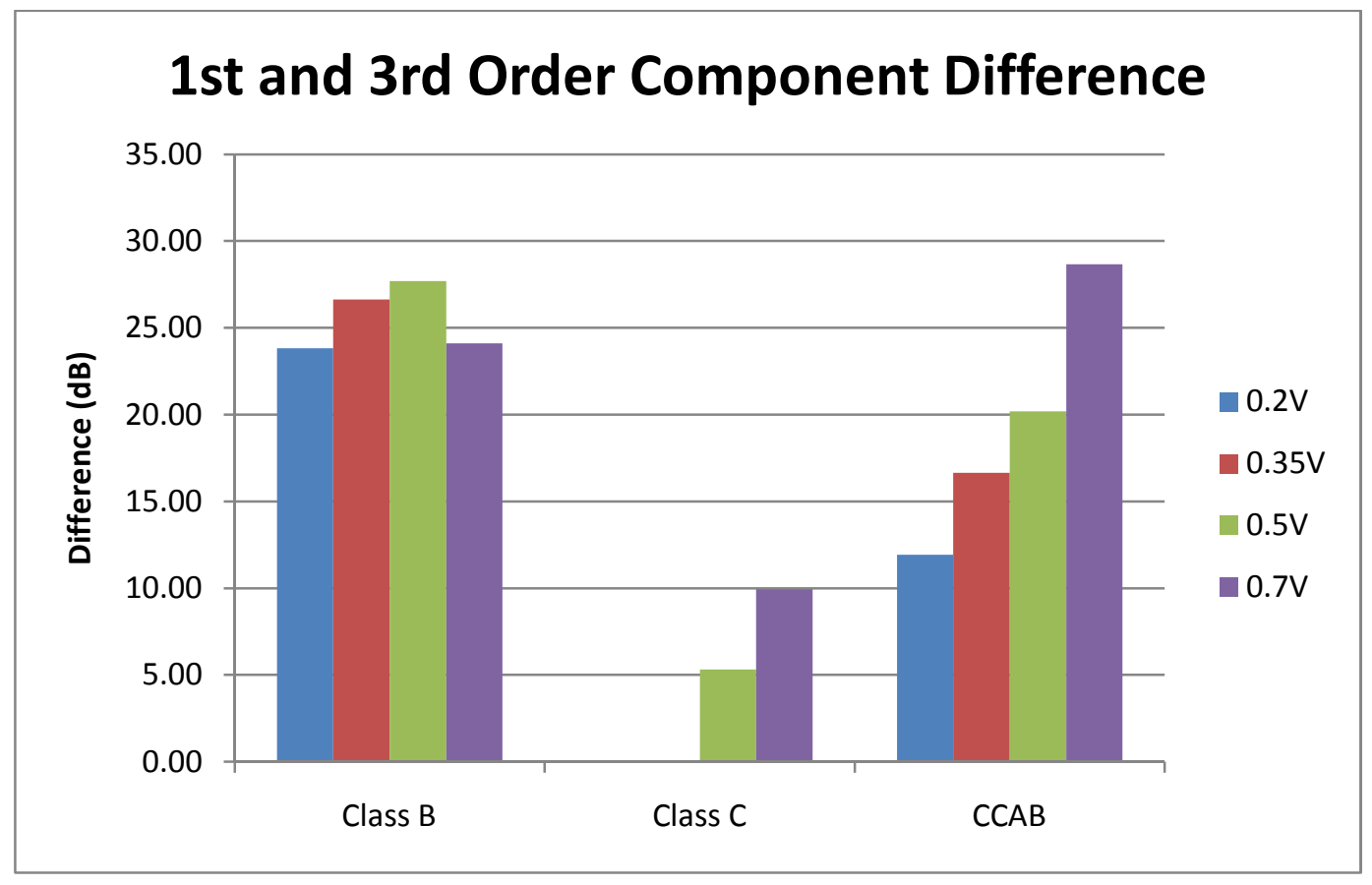

Figure 74: Difference in output power of fundamental and $3^{\text {rd }}$ order intermodulation products for AM input 


\section{Future Steps}

The simulations in this work offer insight on the effectiveness of this CCAB technique for power amplifiers. However, simulations alone are not enough. A physical implementation must be done for this work to be validated. The matching of the transistors in this design is key, which makes implementing this design in discrete parts impractical. Therefore an integrated circuit implementation approach must be taken. The design for this amplifier was done using models from an IBM process offered by MOSIS. Once a license with MOSIS is obtained a layout for the amplifier can be designed, optimized and fabricated. Gain, efficiency, bandwidth, and error vector magnitude measurements should be obtained from this amplifier.

Additionally, this work would benefit from an in-depth analysis of the non-linear feedback provided by the biasing system. An analytical model would allow for selection of the difference amplifier gain to achieve a desired damping factor for the system, rather than the trial and error method used in this design. 


\section{Conclusion}

The proposed CCAB amplifier offered significant improvements in linearity. Dynamic ranges of over $8 \mathrm{~dB}$ were demonstrated. Additionally the CCAB system improved the difference between the fundamental and the $3^{\text {rd }}$ order intermodulation products from $9.9 \mathrm{~dB}$ to $28.7 \mathrm{~dB}$. The effectiveness of this system is limited by the sharpness of the transistor turn on, as well as the change in input impedance to the transistor as the biasing is changed. The ability to achieve class $C$ efficiency with class $B$ linearity makes this amplifier architecture a promising possibility. 


\section{List of References}

${ }^{1}$ Y. Palaskas, "65nm CMOS Power Amplifier" Intel Research, March 2008.

2 J. Liang, B. Ng, J. Chen , and A. Paulraj, "GMSK linearization and structured channel estimate for GSM signals," in MILCOM 97

Proceedings, November 1997, Vol. 2, pp. 817-821.

3 “LTE," 3rd Generation Partnership Project , 2010.

${ }^{4}$ G. Gonzalez, Microwave Transistor Amplifiers, Prentice Hall, 1997.

${ }^{5}$ D. Pozar, Microwave and RF Design of wireless systems, John Wiley and Sons, Inc., 2001.

${ }^{6}$ A. Kavousian, D. Su, M. Hekmat, A. Shirvani, and B. Wooley, "A Digitally Modulated Polar CMOS Power Amplifier With a 20-MHz Channel Bandwidth," IEEE Journal of Solid-State Circuits, Vol. 43, No. 10, October 2008.

${ }^{7}$ F. Raab, "Efficiency of Outphasing RF Power-Amplifier Systems," IEEE Transactions on communications, Vol. 33 No. 10, pp. 1094-1099, October 1985.

${ }^{8}$ J. Jau, Y. Chen, T. Horng, and J. Li, "Envelope Following-Based RF Transmitters Using Switching-Mode Power Amplifiers," IEEE Microwave and Wireless Components Letters, Vol. 16, No. 8, pp. 476-478, August 2006. 
${ }^{9}$ K. Iniewski, Wireless Technologies: Circuits, Systems, and Devices, CRC press, 2008.

${ }^{10}$ R. Gupta, B. Ballweber, and D. Allstot, "Design and Optimization of CMOS RF Power Amplifiers," IEEE Journal of Solid-State Circuits, Vol. 36, No. 2, pp. 166-175, February 2001.

${ }^{11}$ M. Pelgrom, H. Tuinhout and M. Vertregt, "Transistor matching in analog CMOS applications." In IEDM 98 Technical Digest, December 1998, pp. 915-918. 